\title{
WestVirginiaUniversity
}

THE RESEARCH REPOSITORY @ WVU

Graduate Theses, Dissertations, and Problem Reports

2001

\section{Study of the rheological properties of nomex fibrids}

\author{
Long Han \\ West Virginia University
}

Follow this and additional works at: https://researchrepository.wvu.edu/etd

\section{Recommended Citation}

Han, Long, "Study of the rheological properties of nomex fibrids" (2001). Graduate Theses, Dissertations, and Problem Reports. 1193.

https://researchrepository.wvu.edu/etd/1193

This Thesis is protected by copyright and/or related rights. It has been brought to you by the The Research Repository @ WVU with permission from the rights-holder(s). You are free to use this Thesis in any way that is permitted by the copyright and related rights legislation that applies to your use. For other uses you must obtain permission from the rights-holder(s) directly, unless additional rights are indicated by a Creative Commons license in the record and/ or on the work itself. This Thesis has been accepted for inclusion in WVU Graduate Theses, Dissertations, and Problem Reports collection by an authorized administrator of The Research Repository @ WVU. For more information, please contact researchrepository@mail.wvu.edu. 
STUDY OF THE RHEOLOGICAL PROPERTIES OF NOMEX FIBRIDS

Long Han

\author{
A Thesis submitted to the \\ College of Engineering and Mineral Resources \\ at West Virginia University \\ in partial fulfillment of the requirements \\ for the degree of \\ Master of Science \\ in \\ Chemical Engineering
}

Eugene V. Cilento, Ph.D.

Rakesh K. Gupta, Ph.D., Chair

Ray Y. K. Yang, Ph.D.

Department of Chemical Engineering

Morgantown, West Virginia

2001

Keywords: Nomex ${ }^{\circledR}$ Fibrids, Platelet Suspensions, Rheological Properties, suspension viscosity, Einstein Constant, Extensional

Viscosity, Fibrid Morphology 


\section{ABSTRACT \\ STUDY OF RHEOLOGICAL PROPERTIES OF NOMEX FIBRIDS}

\section{Long Han}

Paints and caulks typically contain (spherical) fumed silica particulates as viscosity enhancers. A similar viscosity enhancement can be achieved by using aramid fibrids. Aramid fibrids are believed to form microstructures in suspensions because of the "space-filling" nature of their shapes and thus provide a convenient means of introducing desired rheological behavior for various commercial applications.

The flow responses of aramid fibrids dispersed in mixtures of corn syrup and water were determined in dynamic shear, steady shear, low shear capillary, and extensional stretching flows. Yield stress, strong shear thinning, thixotropy, and strong dispersion effects were observed, and these are consistent with observed equilibrium structures. Intrinsic viscosity measurements revealed Einstein coefficients about 200 times larger than the corresponding value for spheres, and this demonstrates their remarkable viscosity enhancement as well as the strong effect of dispersion. Large Trouton ratios observed during extensional viscosity measurement also prove that fibrids have a tremendous enhancement effect on suspension viscosity. 


\section{ACKNOWLEDGMENTS}

The author wishes to thank Dr. Rakesh Gupta and Dr. Ruifeng Liang for their guidance and help throughout this project. All insight and patience was greatly appreciated.

This project was funded, in part, by the DuPont company. Dr. Deepak Doraiswamy provided me much help and advice during the research. The Nomex ${ }^{\circledR}$ fibrids were supplied by Mr. Dale Reese of DuPont. The author would like to thank the DuPont company for their help.

During the process of the research, the staff of the Department of Chemical Engineering, Mr. James Hall, Mrs. Linda Rogers and Ms. Bonita Helmick, provided much help, the author would like to express his gratitude to them. The author would also like to thank Dr. Eung $\mathrm{Ha}$ Cho for his kind help for loan of the mixing equipment.

The author would like to thank his family, especially his wife, for their support during his master's program. Without the love and support of you all I could not have made it so far. 
TABLE OF CONTENTS

TITLE PAGE I

ABSTRACT II

ACKNOWLEDGMENTS III

TABLE OF CONTENTS IV

LIST OF FIGURES VI

LIST OF TABLES IX

CHAPTER 1 INTRODUCTION 1

1.1 THIXOTROPY AND ITS EXPLOITATION 1

1.2 ARAMID POLYMERS: KEVLAR ${ }^{\circledR}$ SHORT FIBERS AND NOMEX ${ }^{\circledR}$ FIBRIDS $^{2}$

1.3 OBJECTIVE OF RESEARCH AND GENERAL APPROACH

CHAPTER 2 LITERATURE REVIEW 7

2.1 INTRODUCTION

2.2 PARTICULATE SUSPENSIONS

2.3 SHEAR VISCOSITY OF CONCENTRATED SUSPENSIONS OF NON-COLLOIDAL

PARTICULATES

2.4 THIXOTROPY 9

2.5 INTRODUCTION TO SHORT-FIBER SUSPENSIONS 12

2.6 SEMICONCENTRATED FIBER SUSPENSIONS 14

$\begin{array}{lll}2.7 & \text { SUSPENSIONS OF DISKS } & 15\end{array}$

CHAPTER 3 EXPERIMENTAL DETAILS 16

3.1 MATERIAL AND SUSPENSION PREPARATION 16

3.2 SHEAR MEASUREMENT AT FINITE CONCENTRATIONS 18

3.3 GLASS CAPILLARY VISCOMETER MEASUREMENTS 20

3.4 EXTENSIONAL FLOW MEASUREMENTS 23

3.5 MICROSCOPIC OBSERVATION OF DISPERSED FIBRIDS 26

CHAPTER 4 RESULTS AND DISCUSSION 27

4.1 MATRIX FLUID VISCOSITY 27

4.2 STEADY SHEAR VISCOSITY OF FIBRID SUSPENSIONS 29 
4.3 EINSTEIN COEFFICIENT OF FIBRID SUSPENSIONS

4.4 EXTENSIONAL VISCOSITY MEASUREMENT

CHAPTER 5 CONCLUSIONS 52

CHAPTER 6 RECOMMENDATIONS 53

BIBLIOGRAPHY $\quad 54$

APPENDIX A CONFERENCE PUBLICATION 60

$\begin{array}{lll}\text { APPENDIX B SHEAR VISCOSITY DATA } & 64\end{array}$

$\begin{array}{lll}\text { APPENDIX C EINSTEIN COEFFICIENT DATA } & 84\end{array}$

APPENDIX D EXTENSIONAL VISCOSITY DATA 98 
LIST OF FIGURES

FIGURE 1.1 AROMATIC POLYAMIDES 2

FIGURE 1.2 FILMY STRUCTURE OF FIBRID 5

FIGURE 3.1 PHOTOGRAPH IMAGE OF THE CARRI-MED CSL 100 $\begin{array}{ll}\text { RHEOMETER } & 19\end{array}$

FIGURE 3.2 PHOTO IMAGE OF RMS800 19

FIGURE 3.3 CANNON-FENSKE ROUTINE VISCOMETER FOR EINSTEIN $\begin{array}{ll}\text { CONSTANT MEASUREMENT } & 21\end{array}$

FIGURE 3.4 SCHEMATIC DIAGRAM OF THE EXTENSIONAL VISCOMETER

FIGURE 3.5 CONTROL VOLUME USED FOR THE INTERGRAL LINEAR MOMENTUM BALANCE 25

FIGURE 4.1 VISCOSITY DATA FOR CORN SYRUP-WATER SOLUTIONS AT 25 ${ }^{\circ} \mathbf{C}$

FIGURE 4.2 VISCOSITY OF CORN SYRUP WITH DIFFERENT WATER $\begin{array}{lr}\text { CONTENTS } & 28\end{array}$

FIGURE 4.3 GAP SPACING EFFECT FOR 0.18\% F20W IN

CORN SYRUP SOLUTION (23.9 WT\% WATER) 30

FIGURE 4.4 EFFECT OF SOLID LOADING FOR FIBRID IN MATRIX FLUID (13.8\% WATER IN CORN SYRUP) 32 
FIGURE 4.5 EFFECT OF FIBRID SIZE FOR 0.54\% FIBRID

FIGURE 4.6 EFFECT OF MATRIX FLUID VISCOSITY FOR 0.8\% FIBRIDS

FIGURE 4.7 EFFECT OF SOLID LOADING FOR F2OW IN CORN SYRUP

SOLUTION (13.8\% WATER)

FIGURE 4.8 EFFECT OF FIBRID TYPE FOR 0.54\% FIBRIDS IN CORN SYRUP SOLUTION (13.8\% WATER)

FIGURE 4.9 EFFECT OF MEDIUM VISCOSITY FOR 0.54\% F20W IN CORN SYRUP SOLUTION

FIGURE 4.10 COMPARISON OF MODEL FITS WITH EXPERIMENTAL DATA FOR 0.54\% F20W SUSPENSION IN CORN SYRUP SOLUTION $(13.8 \%$ WATER)

FIGURE 4.11 TYPICAL IMAGES OF 0.18\% FIBRIDS DISPERSED IN CORN SYRUP SOLUTION (23.9\% WATER); A: F10W, B: F20W; C: F25W

FIGURE 4.12 DETERMINATION OF EINSTEIN CONSTANT FOR GLASS BEAD IN HYDRAULIC OIL SUSPENSION

FIGURE 4.13 DETERMINATION OF EINSTEIN CONSTANT FOR HOLLOW GLASS BEAD IN HYDRAULIC OIL SUSPENSION

FIGURE 4.14 DETERMINATION OF EINSTEIN CONSTANT FOR HOLLOW GLASS BEAD IN CORN SYRUP SUSPENSION

FIGURE 4.15 DETERMINATION OF EINSTEIN CONSTANT FOR HOLLOW GLASS BEAD IN CORN SYRUP 1 (18\% WATER) SUSPENSION

FIGURE 4.16 DETERMINATION OF EINSTEIN CONSTANT FOR HOLLOW GLASS BEAD IN CORN SYRUP 2 (20\% WATER) SUSPENSION 43 
FIGURE 4.17 DETERMINATION OF EINSTEIN CONSTANT FOR FIBRID IN CORN SYRUP 2 (20\% WATER) SUSPENSION (FINAL RESULTS)

FIGURE 4.18 DETERMINATION OF EINSTEIN CONSTANT FOR FIBRID IN CORN SYRUP 1 (18\% WATER) SUSPENSION (FINAL RESULTS)

FIGURE 4.19 TYPICAL IMAGES OF 100 PPM FIBRIDS DISPERSED IN CORN SYRUP 1 (18\% WATER); A:F10W; B:F20W; C:F25W

FIGURE 4.20 TYPICAL IMAGE OBSERVED DURING THE EXPERIMENT

PROCEDURE A. WITHOUT SUCTION; B. BEFORE SUCTION; C. AFTER SUCTION

FIGURE 4.21 EXTENSIONAL VISCOSITY FOR 200PPM FIBRIDS SUSPENSIONS IN CORN SYRUP SOLUTION (25\% WATER IN WEIGHT) IN COMPARISON WITH SHEAR VISCOSITY

FIGURE 4.22 TYPICAL IMAGES OF 200 PPM FIBRIDS DISPERSED IN CORN SYRUP SOLUTION (25\% WATER IN WEIGHT) A:F10W; B:F20W; C:F25W 
LIST OF TABLES

TABLE 4.1 MEAN VISCOSITY OF CORN SYRUP SOLUTION WITH DIFFERENT CONTENT

TABLE 4.2 DETERMINING THE ACTUAL POLYMER CONTENT IN MOIST POLYMER SAMPLES

TABLE 4.3 COMMON PROPERTIES OF MATRIX SOLVENT

TABLE 4.4 CALCULATION RESULTS FROM BATCHELOR EQUATION FOR EXTENSIONAL VISCOSITY AND RELATIVE VISCOSITY (TROUTON RATIO)

TABLE B1

TABLE C1 - C13 


\section{Chapter 1 Introduction}

\subsection{Thixotropy and its exploitation}

When fine particles are dispersed in low viscosity liquids, the observed flow behavior is the result of interplay between hydrodynamic forces and van der Waals, Coulombic, steric, gravitational and Brownian motion forces. With decreasing particle size, the surface area to volume ratio increases, and surface forces predominate over Brownian motion and gravity. A consequence is that the rest state of the suspension is characterized by the formation of structures - flocs or a lattice. When suspensions are prepared in organic media, Coulombic effects are unimportant and, in the absence of steric stabilizers, the van der Waals forces of attraction make the suspended particles flocculate, trapping liquid and forming a gel. The result is not just high viscosity but also the appearance of an apparent yield stress. This behavior can be exploited for flow control purposes, and fine powders can be employed as thickening agents.

A commercial material that is commonly used to thicken and also reinforce adhesives, sealant, caulks and paints is fumed silica (see, for example, Barthel et al., 1996). Fumed silica is ultra-pure synthetic silicon dioxide that is generally formed by the high temperature hydrolysis of chlorosilanes in a hydrogen/oxygen flame. The result is nonporous, amorphous spheres having a diameter of the order of $10 \mathrm{~nm}$ and surface area exceeding $100 \mathrm{~m}^{2} / \mathrm{g}$. This method of synthesis results in the presence of surface silanol and hydroxyl groups. In a nonpolar liquid, even at loading levels below 5 vol\%, the hydrophilic silica spheres form a network; aggregation into chains is a consequence of interparticle hydrogen bonding.

The strength of the network of fine particles can be changed by changing the amount and chemical nature of the surface groups and also by changing the polarity of the suspending medium. Furthermore, when the gelled suspension is sheared, the floc sizes can be reduced and the network broken down. This reveals itself as severe shear thinning. Upon cessation of shearing, the structure reforms due to Brownian motion, and viscosity builds up again. If the time scale of recovery is large, time dependent effects can arise. In particular, the viscosity can progressively decrease with time even 
when the shear rate is held fixed; this is called thixotropy, (see, for example, Khan and Zoeller, 1993; Yziquel et al., 1999).

Thixotropy is used to advantage in adhesives, paints and building materials. "Thixotropes", such as fumed silica, prevent settling of material during storage, but shear thinning allows for easy spraying and spreading. Structures build up over a 5-30 $s$ time scale, and this prevents sagging and slumping during drying or curing. However, fumed silica suspensions can change their properties over time, and they can also display batch -to -batch variability (Barron, 1996).

\subsection{Aramid polymers: KEVLAR $^{\circledR}$ short fibers and NOMEX ${ }^{\circledR}$ fibrids}

As explained in the previous section, adhesives, sealants and coatings typically contain some fillers that act as viscosity enhancers. These suspensions also tend to be shear thinning and are sometimes thixotropic. There are several options available for achieving the desired rheology: the addition of inorganic fillers such as clays and calcium carbonate, which are not true thixotropes but do increase viscosity; chemically induced thixotropes, such as fumed silica; and fibers such as asbestos and cellulosics, which are mechanical thixotropes. A mechanical thixotrope works by virture of the formation of physical entanglements amongst the fibers, and shear thinning results from flow induced fiber alignment. A replacement for asbestos is the recently introduced aramid short fiber thixotrope by Dupont (Barron, 1996).

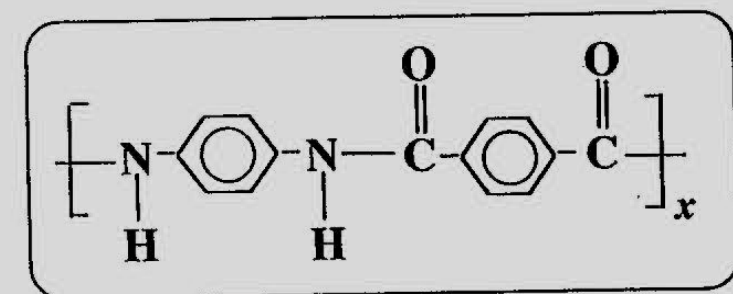

Para-aramid

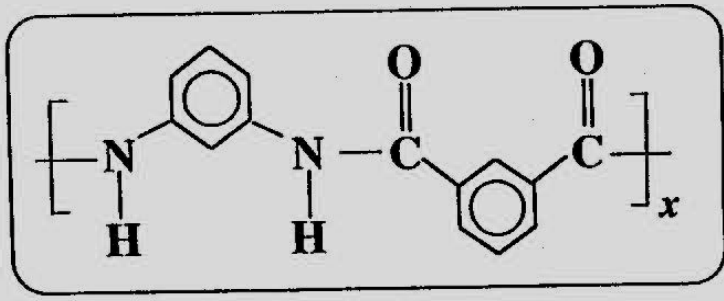

Meta-aramid

(Kevlar)

(Nomex)

Figure 1.1 Aromatic Polyamides 
As seen in figure 1.1, aramids are long chain aromatic polyamides obtained by polymerization of para or meta phenylene diamine and terephthaloyl chloride. The benzene (aromatic) rings serve as the structure's backbone, and their strong molecular bonds provide excellent thermal, mechanical and chemical stability. The para-aramid structure shown on the left is a linear polymer that shows liquid-crystalline character. It is a highly oriented polymer, with outstanding tensile properties, while the meta-aramid chain on the right is less linear and more flexible, leading to excellent mechanical toughness. Fibers of the para-aramid are called Kevlar ${ }^{\circledR}$ by DuPont and are used to reinforce polymer composites while the meta-aramid is marked as Nomex ${ }^{\circledR}$ and employed in fire-resistant, high temperature clothing. Nomex ${ }^{\circledR}$ fibrids are also used in Nomex ${ }^{\circledR}$ paper for high performance electrical insulation where the fibrids act as binder for the fiber component of the paper.

$\operatorname{Kevlar}^{\circledR}$ short fibers are made by solution wet spinning. The fibers are typically 12 microns in diameter and each fiber is surrounded by many smaller fibers called fibrils. Depending upon the product type, the fibers can range in length from less than $0.1 \mathrm{~mm}$ to more than $7 \mathrm{~mm}$, giving a high aspect ratio; this structure also results in a high surface area of 8 to 10 square meters per gram. It is the random orientation and physical entanglement of these fibers and fibrils which results in a high viscosity.

$\mathrm{Kevlar}^{\circledR}$ short fibers have good chemical and solvent resistance. They can also withstand temperature up to almost $500{ }^{\circ} \mathrm{C}$. Due to this reason and because aramid engineered short fibers build thixotropy through mechanical rather than chemical functionalily, adhesives, sealants and coatings incorporating them are more stable over time even when processed at high temperatures than those made with other thixotropes. Liquids containing engineered short fibers will maintain stable, initial viscosity indefinitely, while chemical thixotropes, such as fumed silica, yield lower viscosity over time as competing chemical reactions cause the thixotrope to lose its effectiveness.

As explained by Barron (1996), the nature of the thixotropic mechanism also means that the aramid engineered short fibers can be used broadly, irrespective of the chemistry of the adhesive, sealant or coating or their base resins. This is because, unlike most thixotropes which rely on hydrogen bonding and Van der Waal's forces to 
form associative networks with the polymers and/or solvents in which they are dispersed, the physical network of aramid engineered short fibers is not specific to the matrix system in which it is used, thus providing reliable thickening action irrespective of matrix type.

As opposed to short fibers, fibrids are filmy, ribbon-like particles, currently produced from NOMEX ${ }^{\circledR}$ meta-aramid polymer (poly (isophthaloyl chloride/mphenylenediamine)). Fibrids are formed when a solution of this polymer is dispersed in a coagulant bath. The m-aramid polymer has the thermal and chemical attributes of the $\mathrm{p}$-aramid form but a less linear molecular structure of the $\mathrm{m}$-aramid polymer (see Figure 1.1) and this results in a more flexible and less crystalline material. In contrast to the inherent yellow color of the $p$-aramid polymer, m-aramid products are white.

The term fibrid is derived from the nature of the material, in that it is a fiber-film hybrid, hence fibrid. The filmy structure is illustrated in Figure 1.2. It can be visualized as a white handkerchief that is a few hundred microns or less in both length and width. Fibrids have an extremely uniform and constant thickness of about $0.1 \mu \mathrm{m}$, but the length and width dimensions are variable depending on how the material is processed. The filmy fibrids tend to curl up if not properly handled, so that little "tubes" can result. Varying initial process conditions can also lead to other morphologies and shapes, ranging from beads to foams to stringy particles. Note that the morphology of fibrids gives them an extremely high specific surface area up to $300 \mathrm{~m}^{2} / \mathrm{gm}$. The ratio of length or width to the thickness of the fibrids is also very high, ranging from 3000:1 up to 7000:1. This structure is maintained only in aqueous dispersion and can be lost by improper drying. 


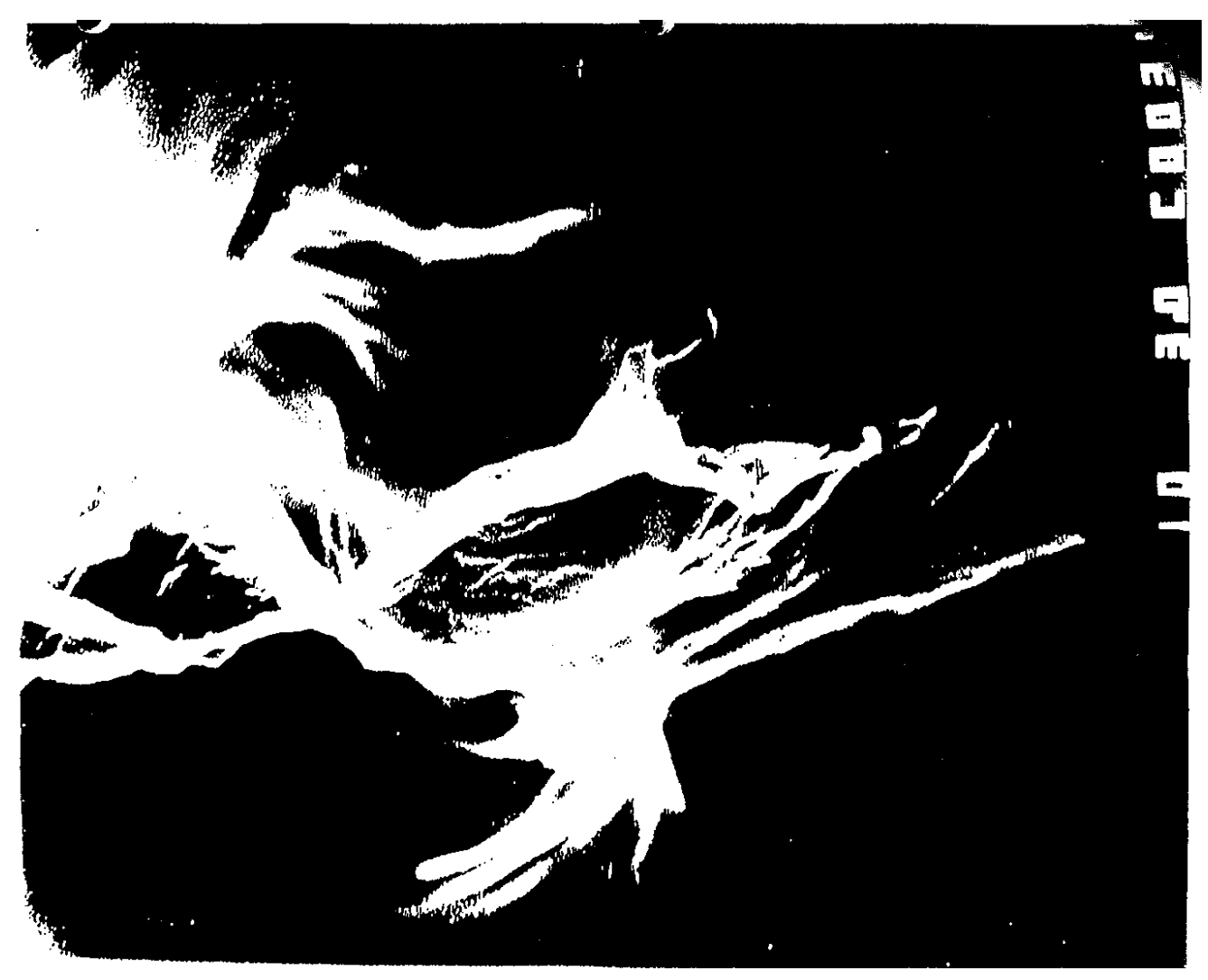

Figure 1.2 Filmy structure of fibrids

\subsection{Objective of research and general approach}

NOMEX ${ }^{\circledR}$ fibrids are a very new product, and the primary goal of this project, funded by the DuPont company, was to understand the mechanism of viscosity enhancement. It was sought to relate fibrid morphology to suspension rheology in both the rest and deformed states. It was also planned to confirm that mechanical rather than chemical interactions were responsible for the thickening action.

As mentioned earlier, fibrid dimensions can be changed by changing the process conditions, and we can obtain fibrids having the same thickness but different lengths. It had been found (by DuPont researchers) that, for the same mass concentration in suspension, increasing the fibrid length did not increase the suspension viscosity in a monotonic manner. Explaining this observation was another goal of this research.

To achieve the above objectives, NOMEX ${ }^{\circledR}$ fibrids of three different aspect ratios were obtained from the DuPont company. These were suspended in mixtures of corn syrup and water at different polymer concentrations and different suspending medium 
viscosities. The suspensions were then subjected to rheological testing in both shear and extension, and these results are described in the chapters that follow. Einstein constant measurements were also made, and they reveal the behavior of the fibrids at infinite dilution. These mechanical measurements were complemented by optical measurements of the fibrids in solution.

Results of this thesis will allow one to get any desired thickener rheology by changing fibrid morphology and fibrid concentration, and this could be achieved at lower thickener loading levels than at present. Another advantage of using fibrids as thickeners is the possibility of obtaining mechanical reinforcement and flame resistance in the final product. The fibrids may also provide barrier properties such as lower gas and moisture diffusivity. 


\section{Chapter 2 Literature Review}

\subsection{Introduction}

There are no data in the literature on suspensions of sheet-like materials since there are encountered infrequently in engineering practice; an example would be mesophase pitch which exists as discotic liquid crystals. Most of the data and associated theories are for particulate suspensions; here the suspended particles are equiaxed, i.e., the dimensions in the three coordinate directions are comparable. Some data are also available for suspensions of short fibers. These two situations are briefly reviewed here in the hope that equations that apply to these data might also describe data on suspensions of sheet-like materials, albeit in an empirical manner. Note, though, that this review is taken entirely from the 2000 book of Professor Gupta entitled, "Polymer and Composite Rheology" $2^{\text {nd }}$ edition and published by Marcel Dekker.

\subsection{Particulate suspensions}

The rheology of suspensions containing rigid fillers is important in many areas of polymer technology. Composite materials containing filler weight fractions in the range of 0.4 to 0.65 are not uncommon, and the fillers may act either as reinforcements or as diluents. A common diluent added to both thermoplastics and thermosets is calcium carbonate, often coated with a stearate. Talc is added to many thermoplastics to increase stiffness and high temperature creep resistance. Most rubber formulations contain carbon black or silica for mechanical property enhancement while rubber is added to polystyrene for the purpose of increasing the impact strength. Concentrated suspensions in polymeric liquids are also encountered in the injection molding of metal powders as well as ceramic powders; here the polymer merely acts as a binder. Additional examples of solid-in-liquid suspensions of technological interest include paints and building materials, coal-water fuels, drilling and fracturing fluids, thermal grease, and dental adhesives. Latices may be suspensions of rigid polymer particles in water, while plastisols are suspensions of polymer particles in a liquid plasticizer. The diversity of applications of suspensions, pastes, and slurries is truly immerse, and this is matched only by the complexity of the rheology exhibited by these materials. Even in 
cases where the suspending liquid is Newtonian in behavior, the presence of a filler produces profound effects on the rheological behvior of the suspension. The rheology becomes even more complex if the liquid phase is non-Newtonian.

Einstein (1956) first predicted the effect of a rigid filler on the viscosity of a Newtonian liquid. His simple equation for the viscosity of dilute suspension is

$$
\eta=\eta_{0}\left(1+K_{E} \phi\right)
$$

The viscosity of the mixture is $\eta$ and that of the suspending liquid is $\eta_{0}$. The volume fraction of filler is $\phi$, and $K_{E}$ is the Einstein coefficient. For particles of spherical shape, $\mathrm{K}_{\mathrm{E}}$ is 2.5. The magnitude of the Einstein coefficient is determined by the degree to which the particles disturb the streamlines in a flowing system. Some particle shapes, such as rods, disturb the streamlines more than do spheres and have correspondingly larger Einstein coefficients; for uniaxially oriented fibers parallel to the tensile stress direction, the Einstein coefficient is $2 L / D$ where $L / D$ is the fiber aspect ratio. Results for prolate and oblate spheroids of different ellipticity values have been derived by Jeffrey (1922). Although the Einstein equation is valid only for very low concentrations of particles, it is amazingly simple. The equation implies that the relative viscosity $\left(\eta / \eta_{0}\right)$ of the suspension is independent of the size or nature of particles. As the solids concentration is increased, particles begin to interact with each other, and the viscosity increases more than linearly with the volume fraction. The coefficient of the quadratic term can be calculated, but its numerical value appears to depend on the assumptions made and the method of calculation used. The results have been summarized by Happel and Brenner (1983).

\subsection{Shear Viscosity of Concentrated Suspensions of Non-Colloidal Particulates}

A very large number of equations has been proposed for estimating the viscosity of a Newtonian liquid containing spherical particles up to moderate concentrations. One of the more successful equations, for monodisperse spheres, is due to Frankel and Acrivos (1967) who assumed that the increase in viscosity on adding particulates was due to energy dissipation in the thin liquid film between neighboring spheres as they moved past each other. These authors further assumed that dissipation due to 
squeezing motion was dominant and that due to the sliding motion was negligible. By averaging the energy dissipated by all possible pairs of particles in the suspension, they obtained the following expression for the relative viscosity (defined as $\eta / \eta_{0}$ ):

$$
\eta_{R}=\frac{9}{8}\left[\frac{\left(\phi / \phi_{m}\right)^{1 / 3}}{1-\left(\phi / \phi_{m}\right)^{1 / 3}}\right]
$$

where $\phi_{\mathrm{m}}$ is the maximum possible solids concentration and is the value of $\phi$ at which the suspension viscosity becomes infinitely large. Equation (2.2) depicts data correctly at large values of $\phi$, but it does not reduce to Equation (2.1) as $\phi \rightarrow 0$.

Several successful empirical equations have emerged from the realization that a unique curve can be obtained by plotting the relative viscosity as a function of $\phi / \phi_{\mathrm{m}}$. Thus, Chong et al. (1971) found

$$
\eta_{R}=\left[1+0.75\left(\frac{\phi / \phi_{m}}{1-\left(\phi / \phi_{m}\right.}\right)\right]^{2}
$$

which does reduce to Equation (2.1) at low values of $\phi$ provided that $\phi_{m}$ equals 0.6 .

The simplest one parameter equation, however, is the one that was evaluated by Kataoka, Kitano and coworkers $(1978,1981)$

$$
\eta_{R}=\left(1-\phi / \phi_{m}\right)^{-2}
$$

and tested extensively by Poslinski et al. (1988) by making room temperature measurements on different concentrations of narrow size distribution glass beads suspended in four different (Newtonian) polybutene matrices.

\subsection{Thixotropy}

Equations (2.1)—(2.4) describe situations where there are particle-fluid interactions but no particle-particle interactions; the latter situation arises for colloidal particles and results in structure formation. This can endow a colloidal suspension with a yield stress and also lead to the phenomenon of thixotropy or reversible rheological changes if the structure can be broken down by flow but structural recovery is not instantaneous; very rapid recovery reveals itself through shear thinning alone. If a suspension is only 
weakly flocculated, the bonds holding the flocs together are weak, and these can be disrupted, in a progressive manner, by flow. On cessation of flow, Brownian motion helps to reestablish the flocculated rest state, although this process may take anywhere from a few minutes to a few hours to be complete. These structural rearrangements manifest themselves in a variety of ways during rheological testing. During shearing at a constant shear rate, for example, the shear stress continues to decrease with time and reaches a constant value only after an extended period of time. If shearing is stopped upon reaching equilibrium but then resumed, the shear stress versus time curve lies below the earlier curve unless a sufficient rest period is given between the two different runs. For the same reason, if the shear rate is ramped up and down, a thixotropic loop is obtained.

Originally the term thixotropy denoted the reversible solid-liquid transition on agitating a gel to convert it to a sol. Today it is used to describe the continuous reduction in viscosity with time of shearing and the subsequent recovery on cessation of flow. Fumed silica in paraffin oil is the prototypical thixotropic system and use is made of this property in the formulation of non-drip paints which contain a polyamidemodified thixotropoic alkyd (Rees, 1995). The amide groups are bound within the alkyd backbone and form hydrogen bonds between themselves; thixotropy arises due to the shear-induced breakdown of these bonds and their slow reformation after the paint has been spread. This results in good leveling properties along with good sag resistance.

A large number of theories have been proposed to mathematically describe thixotropy (Barnes, 1997). In the simplest case, a structure parameter $\lambda$ is used, and it has a value of unity for the completely built-up structure and a value of zero for the completely broken-down structure. These two limits also correspond to the upper and lower Newtonian viscosities, respectively. Under the influence of shearing, there is structure breakdown and recovery, and this process represented by a first order differential equation relating $\mathrm{d} \lambda / \mathrm{d}$ to a term involving breakdown and a term involving buildup; these terms involve only the shear rate and the instantaneous value of $\lambda$. At a given shear rate, the equilibrium value of the structure parameter is determined by setting $\mathrm{d} \lambda / \mathrm{dt}$ equal to zero. The result is a number between zero and unity, and this corresponds to a value of the viscosity between the two Newtonian limits. Thixotropy arises from the time evolution of $\lambda$ as it goes from one equilibrium state to another. 
As mentioned earlier, flocculated suspensions having very vapid structure recovery show a shear viscosity that depends on shear rate. Viscosity models that can portray this behavior have the following general form:

$$
\eta=\eta_{\infty}+\left(\eta_{0}-\eta_{\infty}\right) f(\dot{\gamma})
$$

where $\eta$ is steady shear viscosity; $\eta_{\infty}$ is infinite shear viscosity; $\eta_{0}$ is zero shear viscosity; $\gamma$ is shear rate; $\mathrm{f}(\gamma)$ is a model function depending on shear rate. The Cross model (Cross, 1965) and two forms of the Carreau model (Carreau, 1972) give good agreement with data. These are:

$$
\begin{aligned}
& f(\dot{\gamma})=1 /\left[1+(\alpha \dot{\gamma})^{2}\right]^{(1-n) / 2} \quad \text { Carreau model A } \\
& f(\dot{\gamma})=1 /(1+\alpha \dot{\gamma})^{(1-n)} \quad \text { Carreau model B } \\
& f(\dot{\gamma})=1 /\left[1+(\alpha \dot{\gamma})^{(1-n)}\right] \quad \text { Cross } \bmod e l
\end{aligned}
$$

where $\mathrm{f}(\gamma)$ tends to unity at low shear rate and tends to zero at high shear rates. In these models, $\mathrm{n}$ is the power law index and $\alpha$ is a coefficient which has the dimension of time and is usually considered as a characteristic time constant. There are four model parameters, i.e., $\eta_{0}, \alpha, n, \eta_{\infty}$, with $\eta_{0}$ describing the zero-shear viscosity region, $\alpha$ describing the transition region, $\mathrm{n}$ describing the shear thinning region and $\eta_{\infty}$ describing the high shear rate region. Both $\eta_{0}$ and $\eta_{\infty}$ are determined directly from experimental data of viscosity vs. shear rate, while the other two parameters are determined from the best fit of experimental viscosity vs. shear rate data. 


\subsection{Introduction to short-fiber suspensions}

The addition of short glass, carbon, or aramid fibers to polymers such as nylons and polyesters can result in molded parts having increased toughness, temperature resistance, and dimensional stability. Stiffness and tensile strength also increase with increasing fiber content which can be in excess of $20 \%$ by volume; due to cost considerations, glass is the most common reinforcement. The heat-deflection temperature (HDT) of an unreinforced polymer is typically about $20^{\circ} \mathrm{C}$ below the glass transition temperature; the use of glass reinforcement allows the HDT to easily exceed $100^{\circ} \mathrm{C}$ and to approach the polymer melting point, although there may be a reduction in impact strength. Glass-reinforced thermoplastics can be extruded, thermoformed, injection molded, or blow molded in the conventional way, and they are commonly utilized to make gears and other structural parts. Since they are used in engineering applications, these reinforced plastics are known as "Engineerng Polymers." In shortfiber composites, the fiber length is typically $0.2 \mathrm{~mm}$ while the aspect ration (ratio of length to diameter) is about 15 (Tucker, et al., 1994). Note that fibers having a length ranging from 13 to $25 \mathrm{~mm}$ are also used to make sheet molding compounds and glass mats that are compression molded to produce automobile body panels. Since the glass reinforcement is long and slender, it can be oriented by flow during processing; an extensional flow field is generally more effective compared to a shear field for this purpose. The fiber orientation gets frozen into the solid composite and makes the mechanical properties of the final part be anisotropic.

A discussion of the flow behavior of suspensions containing $\mathrm{n}$ fibers per unit volume, with each fiber having length $L$ and diameter $D$, is logically divided into at least three concentration regimes. At one extreme, in the dilute region, individual fibers can rotate freely without encountering other fibers, and this makes the suspension viscosity cycle at the same frequency. This requires that $n$ be less than $1 / L^{3}$. At the other extreme, in the concentrated region, "logjams" can develop and the suspension behaves more like a solid than as a liquid. This happens when the spacing between fibers becomes of the same order as the fiber diameter. For random orientation of the fibers, this requires that $n$ approach $1 / \mathrm{DL}^{2}$, while, for fibers lying parallel to each other, $n$ can be large and equal $1 / D^{2} L$ (Dinh and Armstrong, 1984). Since the fiber volume fraction, $\Phi$, is of the order of 
$n D^{2} \mathrm{~L}$, the semiconcentrated region for randomly oriented fiber suspensions is defined by:

$$
\left(\frac{D}{L}\right)^{2}<\phi<\left(\frac{D}{L}\right)
$$

and the region boundaries depend explicitly and strongly on the aspect ratio of the fibers. We will neglect wall effects and assume that the fiber dimensions are such that Brownian motion can be neglected. Also we will assume that while the fiber orientation can change during flow, the fibers are always uniformly distributed within the suspension. In reality, we often observe fiber clustering, fiber migration, and the presence of air-filled voids (Wu, 1979; Becraft and Metzne, 1992). Fiber migration is caused by the presence of normal stress gradients, while fiber clustering is exascerbated by increasing the loading level and by decreasing the deformation rate.

Data on fiber suspensions are much less extensive and much less definitive compared to data on particulate suspensions. This is due to the difficulty of obtaining reliable and repeatable results on well characterized fiber suspensions. Although some of the problem can be traced to fiber dimensions being comparable to typical viscometer gaps, fiber flexibility, and the occurrence of mechanical degradation (breakage) of fibers during compounding and viscosity measurement (Vaxman, et al., 1989), other problems arise due to the simple fact that the suspended fibers are oriented by flow. During capillary rheometry, for example, it is not surprising to find that fibers are randomly oriented at low shear rates, but get highly aligned in the flow direction at high shear rates (Crowson, 1980A). However, at a fixed shear rate, the extent of orientation increases as the capillary length decreases. This happens because fibers actually get aligned in the converging flow region at the die entrance, and this orientation is gradually lost during shear flow in the capillary; the loss of orientation with increasing capillary length is most apparent at low shear rates. Similarly but more strikingly, during diverging flow as happens after entry into a mold, fiber alignment build up at mold entrance is lost extremely rapidly, and it is replaced with fiber alignment in a direction perpendicular to the flow direction. As might be expected, these changes in fiber orientation manifest themselves as changes in fluid rheology. 
The above observations can be employed to qualitatively explain the observed viscosity behavior of short fiber suspensions. It is found that the steady shear viscosity of a polymeric liquid, as measured with a capillary viscometer, is increased upon increasing either the fiber length or the fiber concentration, with fiber concentration being the more important of the two variables. The increase can be appreciable but only at low shear rates; at high shear rate, the suspension and suspending liquid viscosity are virtually identical (Crowson, 1980B). At low shear rates, since the fibers are randomly oriented, liquid is essentially forced to flow through a fiber mat, and this results in a high viscosity. At high shear rates, on the other hand, fiber alignment leads to an unsheared plug near the capillary axis and a significantly reduced resistance to flow. The presence of the unsheared plug also results in a blunt velocity profile, and this is observed as shear thinning even at shear rates where the suspending liquid has a constant viscosity. The effect of fiber addition on the activation energy for flow is found to be small, but the addition of fibers whose length is greater than twice the capillary diameter results in pressure fluctuations. At high fiber loading levels, the suspension can become solid-like and exhibit a yield stress (Bennington, et al., 1990). In general, the rheological properties of fiber suspensions depend on variables like the fiber volume fraction, fiber aspect ratio and its distribution, deformation rate, temperature, suspending medium rheology, and the nature of the flow field.

\subsection{Semiconcentrated fiber suspensions}

It is easiest to consider the behavior of semiconcentrated suspensions in extensional flow, since one may assume perfect fiber alignment in the stretch direction at steady state. This was done by Batchelor, who used a cell model to determine the stress field around a fiber of interest when the average distance between fibers was much less than the fiber length but much greater than the fiber diameter (Batchelor, 1971). The result for the suspension extensional viscosity is:

$$
\eta_{E}=\eta\left[3+\frac{4 \phi(L / D)^{2}}{3 \ln (\pi / \phi)}\right]
$$

Equation (2.10) was successfully put to a test by Mewis and Metzner, who carried out fiber-spinning experiments using $0.1-1$ vol \% glass fibers suspensed in a low- 
molecular-weight polybutene (Mewis and Metzner, 1974). These authors found that the extensional viscosity of their fiber suspensions was independent of stretch rate, as predicted by equation (2.10). Extensional viscosity values that were as many as 260 times larger than the suspending oil viscosity could be quantitatively explained by Eq. (2.10), which also correctly portrayed the separate influence of fiber concentration and fiber aspect ratio. Sridhar and Gupta measured tensile stresses down the spinline for the stretching of glass fibers suspended in a mixture of kerosene and polybutene, and found that the Batchelor theory gave numerically correct results only near the spinneret; stresses increased by as much as an order of magnitude further down the spinline (Sridhar and Gupta, 1986). These authors hypothesized that this increase in stress was the result of decreasing interfiber distance, and they provided a simple correction to the Batchelor equation to account for this phenomenon. The influence of distribution of fiber aspect ratios has been taken into account by Pittman and Bayram (1990), while Goddard has considered the effect of non-Newtonian behavior of the suspending medium (Goddard, 1976A, 1976B, 1978). The use of a shear-thinning liquid as the suspending medium is predicted to result in a greatly diminished fiber contribution to the measured stress as compared to the Newtonian case.

\subsection{Suspensions of disks}

A disk is defined as a particle in which two of the linear dimensions are comparable in size and are much larger than the third dimension. A disk is also characterized by a large aspect ratio. Experimental data on discotic suspensions are scarce, and only some flow simulations on suspensions of disks have been published (Gautier, et al., 1999). Adding disks to a liquid, even at a small volume fraction, is able to greatly enhance the flow resistance of the material, provided that the aspect ratio of each particle is large. This is because a disk has a substantial influence on transport fields throughout a spherical region of its largest linear dimension. But the important distinction between transport in dispersions of disks and fibers arises through differences in the form of the disturbance field. The disturbance caused by a disk and disk-disk interactions are much stronger than the disturbance caused by a fiber and the fiber-fiber interactions (Sundararajakumar, et al., 1994). 


\section{Chapter 3 Experimental Details}

\subsection{Material and suspension preparation}

Three NOMEX® fibrids with different aspect ratios but same mass density of 1.38 $\mathrm{g} / \mathrm{cm} 3$ were used in this work, and these are referred to as F10, F20 and F25 as per DuPont terminology; F10 are standard unrefined fibrids, and the increasing number reflects increasing levels of mechanical work done on the fibrids in a refining process, leading to smaller dimensions and aspect ratios. Thus the aspect ratio for these fibrids was supposed to have the following relationship: F10W > F20W > F25W. These fibrids were provided by Dupont in the form of white wet aggregates with varying water content because structure collapses on drying. F10 aggregates were found to contain $\sim 85.2 \mathrm{wt} \%$ water, F20 aggregates $\sim 79.9 \mathrm{wt} \%$ water, and F25 aggregates $\sim 77.3 \mathrm{wt} \%$ water. The actual water content of each fibrid was determined each time before preparing master suspensions, and this was taken into account when calculating polymer concentration.

To prepare finite concentration suspensions, the fibrids were suspended in a commercial grade corn syrup (with a viscosity of 3.91 Pa.s and mass density of $1.382 \mathrm{~g} / \mathrm{ml}$ ) to form a master suspension at a high concentration $(1.2 \mathrm{wt} \%)$; the corn syrup was diluted with different amounts of water to obtain varying levels of Newtonian suspending liquid viscosity $(0.74,0.245$ and $0.07 \mathrm{~Pa}$.s for water concentrations in corn syrup of $7.1,13.8$ and $23.9 \%$, respectively at $25^{\circ} \mathrm{C}$ ). Here the corn syrup used was Karo's light corn syrup produced by CPC International Inc., Englewood Clifts, NJ and purchased from a local K-Mart grocery store. Three fibrid concentrations within a practical range $(0.18 \%, 0.54 \%$ and $0.80 \%$ by weight) were considered for each fibrid type and suspending liquid viscosity level so that a total of 27 formulations were prepared and used for rheological characterization in shear flow.

Fibrid morphology is strongly influenced by the state of dispersion, and it is critical that fibrids be properly dispersed during suspension preparation. In order to facilitate dispersion, use was made of the high viscosity of pure corn syrup: the fibrid aggregates were separated by hand and then pre-dispersed in pure corn syrup, using an Arrow 1750 motorized stirrer for 3 minutes. The dispersion quality was verified by visual 
inspection under a microscope. A master batch suspension of $1400 \mathrm{~g}$ was prepared for each type of fibrid from which the various test suspensions were prepared in $150 \mathrm{~g}$ quantities by diluting with water and corn syrup by high shear stirring for 2 minutes. The prepared suspensions were allowed to rest for one week to eliminate air bubbles; and no detectable sedimentation or phase separation was observed during this time. Still, to be on the safe side, the suspensions were mildly stirred before loading samples for measurements.

It was found that morphological and rheological results were different at finite concentrations and infinite dilution concentrations due to different dispersion intensities involved. To prepare suspensions at infinite dilution, the fibrid aggregates were first dispersed at a concentration of about $0.15 \mathrm{wt} \%$ in pure corn syrup (with a viscosity of 4.162 Pa.s in this batch) using the Arrow stirrer for 15-20 minutes to form a master suspension of $150 \mathrm{~g}$. From this master batch suspension, each type of fibrid suspension at concentrations of 100,200,300, and 400ppm was prepared in $150 \mathrm{~g}$ quantities by thinning down with water and corn syrup by high shear stirring for 1 minute. Two slightly different media were used with viscosities of 0.1612 Pa.s and 0.1156 Pa.s corresponding to $18 \%$ and $20 \%$ water in corn syrup, giving densities of $1.313 \mathrm{~g} / \mathrm{ml}$ and $1.306 \mathrm{~g} / \mathrm{ml}$, respectively. Time was also allowed for removal of air bubbles. Relative viscosity measurements were then made employing a glass capillary viscometer.

Hollow glass bead suspensions were also prepared and used to obtain the expected Einstein coefficient for spheres when working with a glass capillary viscometer. Hollow glass beads with an average diameter of $11 \mu \mathrm{m}$ and mass density of $1.128 \mathrm{~g} / \mathrm{cm} 3$ were dispersed at (i) concentrations ranging from 0.6 to $2.4 \mathrm{wt} \%$ in a mineral oil with a viscosity of 0.1046 Pa.s and mass density of $0.881 \mathrm{~g} / \mathrm{ml}$, and (ii) concentrations ranging from 1.2 to $4.8 \mathrm{wt} \%$ in corn syrup/ water solutions with viscosities of 0.1612 Pa.s to 0.1156 Pa.s. Experiments were also done with suspensions of solid glass beads having an average diameter of either $4 \mu \mathrm{m}$ or $11 \mu \mathrm{m}$.

For extensional flow measurements, $1500 \mathrm{~g}$ of a $200 \mathrm{ppm}$ suspension was prepared for each type of fibrid. For the best dispersion, again, weighed fibrids were dispersed first in pure corn syrup by high shear stirring using the Arrow stirrer for 20 minutes and 
then diluted with appropriate amount of water by high shear stirring for 3 minutes. The medium chosen had a viscosity of 0.051 Pa.s and density of $1.262 \mathrm{~g} / \mathrm{ml}$, corresponding to $25 \%$ water in corn syrup. Here a lower medium viscosity was used in order to form a stable filament for extensional flow measurements.

\subsection{Shear measurement at finite concentrations}

Two different instruments were employed to make shear flow measurements on fibrid suspensions at finite concentrations. These were a Carri-Med CSL 100 rheometer and a Rheometric Scientific RMS 800 rheometer; photographs of these two viscometers are shown in Figures 3.1 and 3.2, respectively. Note that the RMS 800 instrument is a much more versatile instrument and a much more complex instrument as compared to the CSL 100. This rheometer was operated by Dr. R. Liang and additional details are available in a joint manuscript that has been prepared for publication.

Steady shear data were obtained at $25^{\circ} \mathrm{C}$ on the RMS 800 fitted with a fluids transducer giving a reliable minimum torque of $0.001 \mathrm{~g} . \mathrm{cm}$ while working with a pair of $50 \mathrm{~mm}$ diameter parallel plate fixtures. Gap (bridging) effects were effectively eliminated by moving to large gaps (a $1.5 \mathrm{~mm}$ gap was used). Evaporation effects were minimized by coating the rim with low viscosity Wesson vegetable oil. It was found that this thinlayer coating of oil also helped to prevent the test sample from being squeezed out of the parallel plate fixtures when shearing at high rates. Loading and initial structure effects were eliminated by subjecting the sample to multiple strain sweeps (2-200\%) at a fixed frequency of $1 \mathrm{rad} / \mathrm{s}$.

The Carri-Med instrument could be used to measure the shear viscosity only in a narrow shear rate range between 40 and 120 1/s. Parallel plate fixtures having a diameter of $4 \mathrm{~cm}$ and a gap separation of $1000 \mu \mathrm{m}$ were used. 


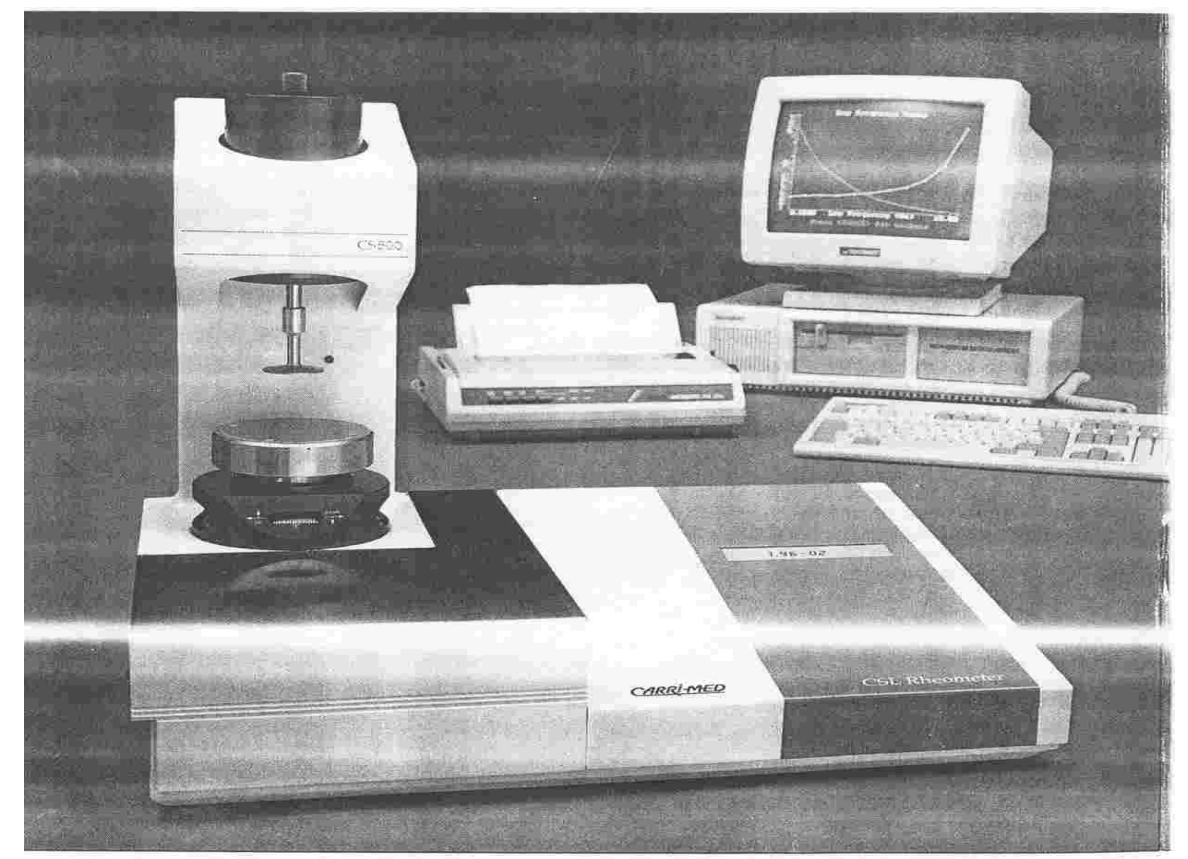

Figure 3.1 Photograph image of The Carri-Med CSL 100 Rheometer

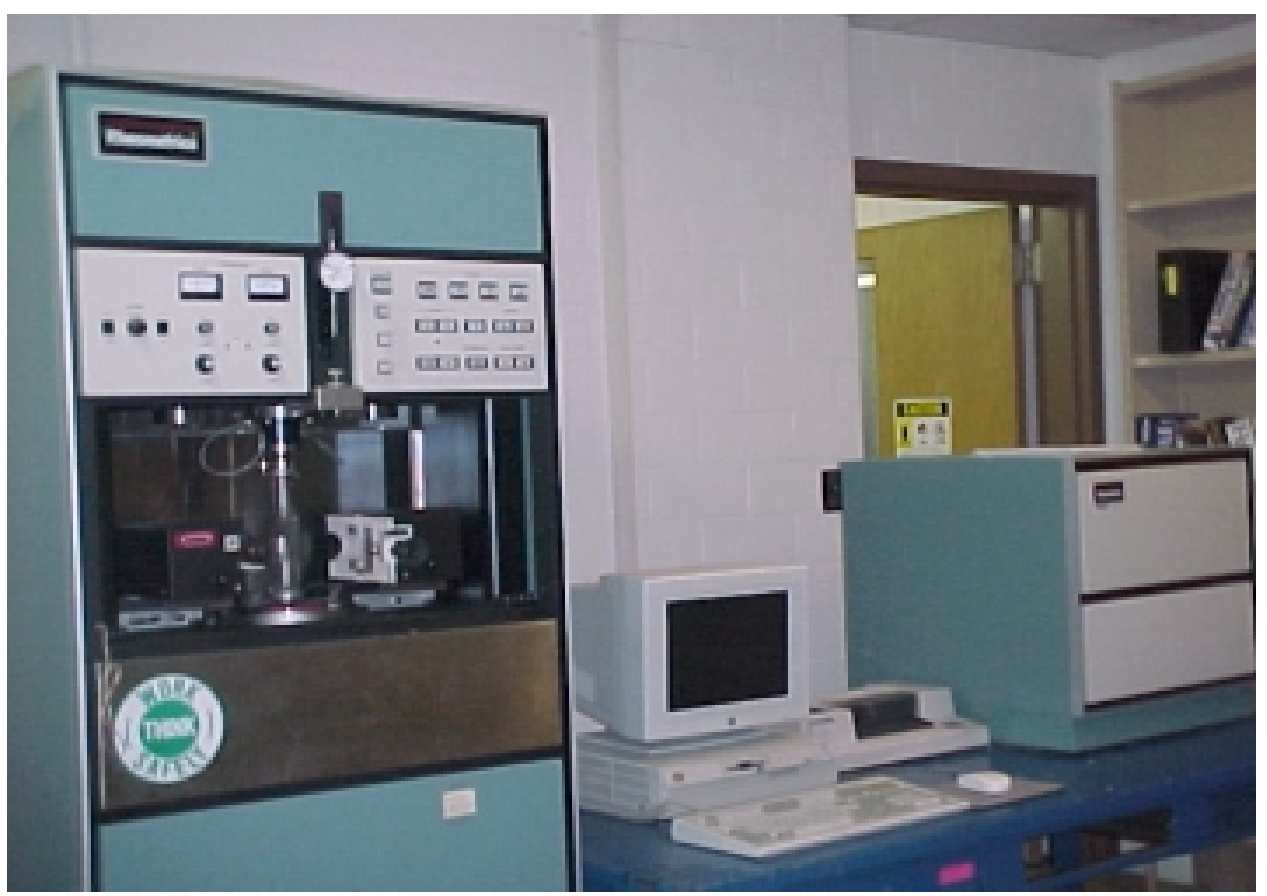

Figure 3.2 Photo image of RMS800 


\subsection{Glass capillary viscometer measurements}

Glass capillary viscometer measurements were carried out on a Cannon-Fenske Routine Viscometer (shown in Figure 3.3) at $25^{\circ} \mathrm{C}$. A viscometer of size 500 was used for high viscosity pure corn syrup and a viscometer of size 300 was used for fibrid suspensions. Note that a viscometer of size 500 can be used to measure fluid kinematic viscosity in the 1600 to $8000 \mathrm{~mm}^{2} / \mathrm{s}$ range while size 300 is used in the 50 $250 \mathrm{~mm}^{2} / \mathrm{d}$ range. Each measurement consisted of the following steps: cleaning the viscometer using solvents such as chromic acid, water, and acetone, and drying the viscometer using clean, dry, filtered air; then charging the sample into the viscometer and holding it in a constant -temperature bath at $25^{\circ} \mathrm{C}$; followed by measuring the efflux time for the sample to flow between two marks of the test section of the capillary; finally repeating the measurement for the same sample. The relative viscosity of each suspension was determined from the efflux or drainage time of the suspension and that of the dispersion medium.

Measurement procedures are carried out in a capillary viscometer of Size 500\& 300 . The detail procedures are as follows:

1. Clean the viscometer using suitable solvents, and by passing clean, dry, filtered air through the instrument to remove the final traces of solvents. Periodically, traces of organic deposits should be removed with chromic acid or non-chromium cleaning solution.

2. To charge the sample into the viscometer, invert the instrument and apply suction to tube $G$, immersing tube $A$ in the liquid sample, and draw liquid to mark $E$. Wipe clean arm $A$, and turn the instrument to its normal vertical position. (Apply suction to tube $A$, immersing tube $G$ in the liquid sample, draw liquid to link position of tube $G$ and larger bulb.)

3. Place the viscometer into the holder, and insert it into the constant temperature $\left(25^{\circ} \mathrm{C}\right)$ bath. Align the viscometer vertically in the bath.

4. Allow liquid flow down along tube $A$ through mark $C$, then begin to measure the efflux time when the meniscus comes to mark $C$. For size 300 viscometer, this period of time, or equilibrium time, is about 4 mins. (Allow approximately 10 minutes for the 
sample come to the bath temperature. Then apply suction to tube A, draw the liquid slightly above the upper mark C.)

5. To measure the efflux time, allow the liquid sample to flow freely down past mark $C$, measuring the time for the meniscus to pass from mark $C$ to mark $E$.

6. A check run may be made by repeating the above steps.

During the measurement, in order to obtain reproducible results, attention was paid to minimizing settling of fibrid particles, strictly maintaining the bath at the testing temperature, carefully holding the viscometer in a vertical position, and accurately charging the same amount of sample into the viscometer every time. Finally, the difference in drainage time was found to be less than $0.5 \mathrm{~s}$ between two runs for the same suspension.

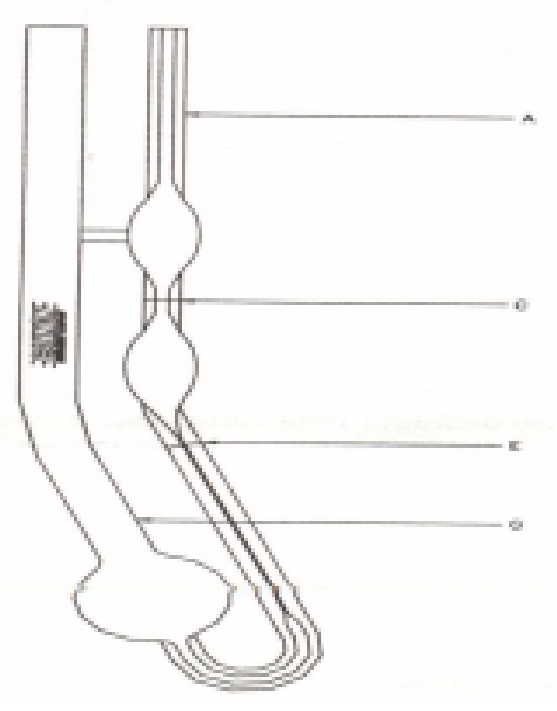

Figure 3.3 Cannon-Fenske Routine Viscometer for Einstein Constant Measurement

Suspension preparation is the key point to obtain the accurate results. Whether the solid has been dispersed evenly in solution or not is the criterion for determining the stirring time.

During this series of experiments, several different suspensions were prepared. They are: Glass Beads in Corn Syrup, Glass Beads in Hydraulic Oil, Hollow Glass 
Beads in Hydraulic Oil, Hollow Glass Beads in 18\% corn syrup, Hollow Glass Beads in $20 \%$ corn syrup, and Fibrids in $18 \%$ \& $20 \%$ corn syrup.

In contrast to fibrids suspensions, glass bead or hollow glass bead suspensions in any of the matrix fluids is much easier to prepare. The common steps are as follows,

1. To prepare the master solution, add specific amount of solids and matrix fluid and stir for 10 minutes at level 4 of stirrer.

2. To prepare the suspension to be measured, add specific amount of master solution and corresponding amount of matrix fluid to prepare the suspension of specific concentration. Stir at level 2 for 1 minute.

3. For matrix fluid of corn syrup, keep the suspension in room temperature for about 4 days before the measurement and let the air bubbles inside the solution disappear. For all other matrix fluids, just keep the suspension in room temperature for about 6 hours, then the suspension is ready for measurement.

For the system of fibrids in $18 \%$ \& $20 \%$ corn syrup, the suspension preparation procedure is slightly different. Since the polymer sample is wet, we first need to determine the actual polymer content in the polymer sample. The steps for preparing the solution are as follows,

1. Prepare the master solution by adding specific amounts of fibrids and corn syrup. Stir at level 4 for about 15 20 minutes. This time depends on whether any clusters of fibrids remain in the solution. For accurate measurement, the fibrids must be dispersed evenly. F10W was found to be the most difficult to disperse by stirring.

2. Prepare the suspension to be measured by adding specific amount of master solution and corresponding amount of corn syrup and water to prepare the suspension of specific concentration. Stir at level 2 for 1 minute.

3. Keep the suspension at room temperature for about 4 days, and then the suspension is ready for use.

Note: During solution preparation, the purpose of beginning with fibrids and corn syrup for the master solution is to make the fibrids disperse most evenly. Then adding 
the specific amount of corn syrup and water into master solution gives the solution of desired concentration.

Table C1, C2 and C3 gives the details about preparing the master solutions and suspensions for F10W, F20W and F25W in corn syrup 1 (18\% water in corn syrup).

\subsection{Extensional flow measurements}

A schematic diagram of the extensional viscometer is shown in figure 3.4. Fluid is pumped into a jacketed cylindrical tank $C$ and leaves through the capillary $E$; the volumetric flow rate is kept constant with the help of a pump. If the liquid were Newtonian and had a viscosity $\mu$, the pressure drop versus flow rate relation across capillary E would be

$$
Q_{0}=\frac{\pi d^{4}}{128 \mu L}\left[P_{0}+\rho g L\right]
$$

where $P_{0}$ is the pressure at the capillary inlet, $d$ is the capillary diameter and $L$ is the capillary length. Here d was $2.896 \mathrm{~mm}$ and $\mathrm{L}$ was $5 \mathrm{~cm}$.

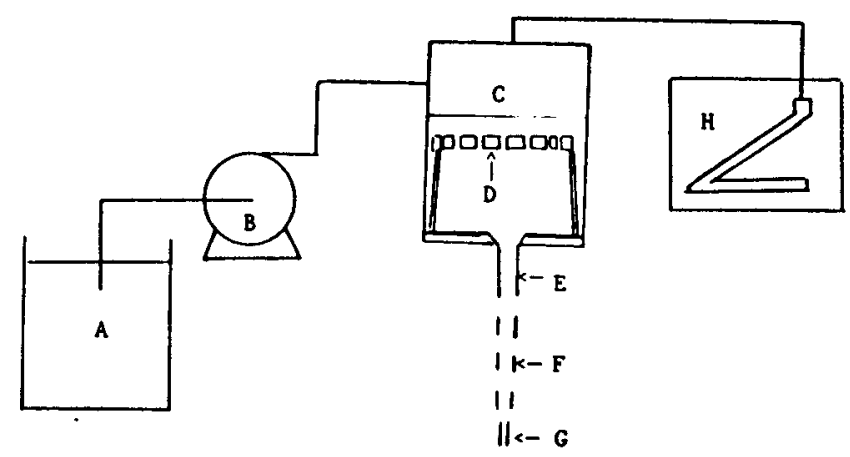

\footnotetext{
A Fluid Reservoir

B Pump

C Viscometer

D Baffle
}
E Upper Capillary
F Filament
G Lower Capillary
H Manometer

Figure 3.4 Schematic diagram of the extensional viscometer 
The liquid jet leaving capillary E can be stretched by sucking the liquid into another capillary G (Khagram et al., 1985); average axial liquid velocity increases progressively in the air gap between capillaries $E$ and $G$. Fluid stretching results in the application of a tensile stress $T_{1}$ at the exit of capillary $E$. As a consequence, the pressure drop versus flow rate relation across $E$ becomes

$$
Q_{1}=\frac{\pi d^{4}}{128 \mu L}\left[P_{1}+T_{1}+\rho g L\right]
$$

where $P_{1}$ is now the pressure at the inlet to $E$. Since at steady state, $Q_{0}$, equals $Q_{1}$, it must be true that (Sridhar and Gupta, 1985)

$$
T_{1}=P_{0}-P_{1}
$$

It can easily be shown that $\left(\mathrm{P}_{0}-\mathrm{P}_{1}\right)$ equals the reduction in air pressure above the liquid in tank $C$ (Chan et al., 1988). This change in pressure is measured using a water manometer $\mathrm{H}$ having one arm at a $5^{\circ}$ angle to the horizontal.

Even though Eqs. (3.1) - (3.3) have been shown to be valid for Newtonian liquids, Eq. (3.3) holds for non-Newtonian liquid also if the arguments are repeated using the power-law model to represent the fluid rheology. Thus, it is a straightforward matter to measure the tensile stress in the liquid at the exit of the capillary E. Multiplying this stress by the cross-sectioned area of the filament at the capillary exit gives the stretching force $F(0)$ shown in figure 3.5. A knowledge of $F(0)$ then allows us to calculate $F(1)$, the tensile force in the stretching liquid, at any other axial position $X$, by means of a force balance (see figure 3.5):

$$
\rho \int_{C V} V(x)(\underset{\sim}{V} \bullet d \underset{\sim}{A})=\sum F
$$

If we neglect air drag, the above equation becomes

$$
F(1)=F(0)+F(I)-F(S T)-F(G)
$$

where $F(1)$ is the desired force at $X_{1}$ and

$$
\begin{aligned}
& F(0)=\left(P_{0}-P_{1}\right) * \text { cross sectioned area at } \mathrm{X}_{0} \\
& F(S T)=\pi \gamma\left(D_{1} \cos \theta_{1}-D_{0} \cos \theta_{0}\right), \text { the surface tension force }
\end{aligned}
$$


$F(I)=\rho\left(\int_{A_{1}} V^{2} d A-\int_{A_{0}} V^{2} d A\right)$, the force due to inertia

$F(G)=\rho g \int_{X_{0}}^{X_{1}} \pi r^{2}(\xi) d \xi$, the gravity force

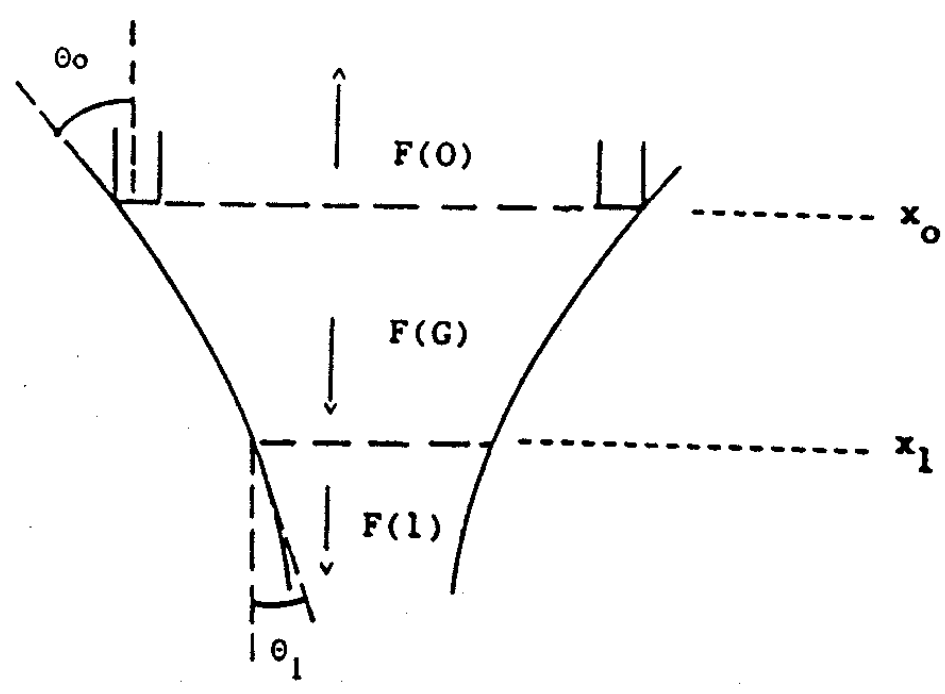

Figure 3.5 Control volume used for the intergral linear momentum balance

In which the coefficient of surface tension $\gamma$ is taken to have the value $0.07 \mathrm{~N} / \mathrm{m}$.

The ratio of the force $F(1)$ to the cross-sectioned area at $X_{1}$ gives the viscoelastic stress in the axial direction $\sigma_{11}\left(X_{1}\right)$. A radial force balance at $X_{1}$ gives the radial stress $\sigma_{22}$ in the fluid, and the difference between $\sigma_{11}$ and $\sigma_{22}$ can be shown to be (Chan et al., 1988)

$$
\sigma_{11}-\sigma_{22}=\sigma_{11}+\gamma / R
$$

where $\mathrm{R}$ is the filament radius at any location.

Finally, the extensional viscosity $\eta_{E}$ is defined as

$$
\eta_{E}=\frac{\sigma_{11}-\sigma_{22}}{d u / d x}
$$


in which $\mathrm{du} / \mathrm{dx}$ is the stretch rate obtained by differentiating the axial liquid velocity with respect to the axial distance. For a constant volumetric flow rate $Q$ and assuming no radial variation in the velocity, the local velocity and stretch rate $\varepsilon$ are given by

$$
u=Q /\left(\pi R^{2}\right)
$$

and

$$
\dot{\varepsilon}=-2 Q(d R / d x) /\left(\pi R^{3}\right)
$$

where the radius $R$ was measured with the help of a high performance CCD camera. The range of stretch rates could be changed by varying the flow rate $Q$ and by altering the distance between the upper and lower capillaries.

\subsection{Microscopic observation of dispersed fibrids}

Fibrid structures and the state of dispersion of fibrids were examined on a Micromaster optical microscope equipped with a COHU high performance CCD optical zoom camera in terms of semi-quantitative image analyses and correlated with the results obtained from the RMS 800 rheometer, the glass capillary viscometer and the extensional viscometer. Microscopic observation was also conducted and used to optimize the stirring conditions to ensure that no obvious clusters of fibrids remained and that most fibrids were evenly dispersed during the process of suspension preparation. 


\section{Chapter 4 Results and Discussion}

\subsection{Matrix fluid viscosity}

Mixtures of corn syrup and water were used to suspend the Nomex $^{\circledR}$ fibrids examined in this work. To relate the suspension viscosity to the suspending liquid viscosity, the shear viscosity of the store-bought Karo's light corn syrup was measured as a function of the amount of added water; the CSL 100 rotational viscometer, fitted with $4 \mathrm{~cm}$ diameter parallel plates and employing a gap of $1000 \mu \mathrm{m}$ was used for this purpose. Results of measurements at five different water contents are plotted in Figure 4.1 as a function of shear rate. As expected, the viscosity at a given concentration is constant, independent of shear rate, and average viscosity values are listed in Table 4.1. These data are also displayed in figure 4.2 as (Newtonian) viscosity versus weight percent of added water. Clearly, the viscosity is very sensitive to water content at low water contents. The measurement temperature was $25^{\circ} \mathrm{C}$. This temperature was chosen because it was slightly higher than the temperature of the laboratory, and it was easy to keep the samples at this temperature during measurement. Note that the range of shear rates chosen was based on the measurement range of the torque transducer. Figure 1 in Appendix $B$ shows that going below $40 \mathrm{~s}^{-1}$ did not give reliable results.

TABLE 4.1 MEAN VISCOSITY OF CORN SYRUP SOLUTION WITH DIFFERENT CONTENT

\begin{tabular}{|c|c|c|}
\hline No. & Weight \% water & Mean Viscosity (poise) \\
\hline 1 & 0 & 49.7 \\
\hline 2 & 2.05 & 28.3 \\
\hline 3 & 7.1 & 9.2 \\
\hline 4 & 13.8 & 2.5 \\
\hline 5 & 23.9 & 0.69 \\
\hline
\end{tabular}




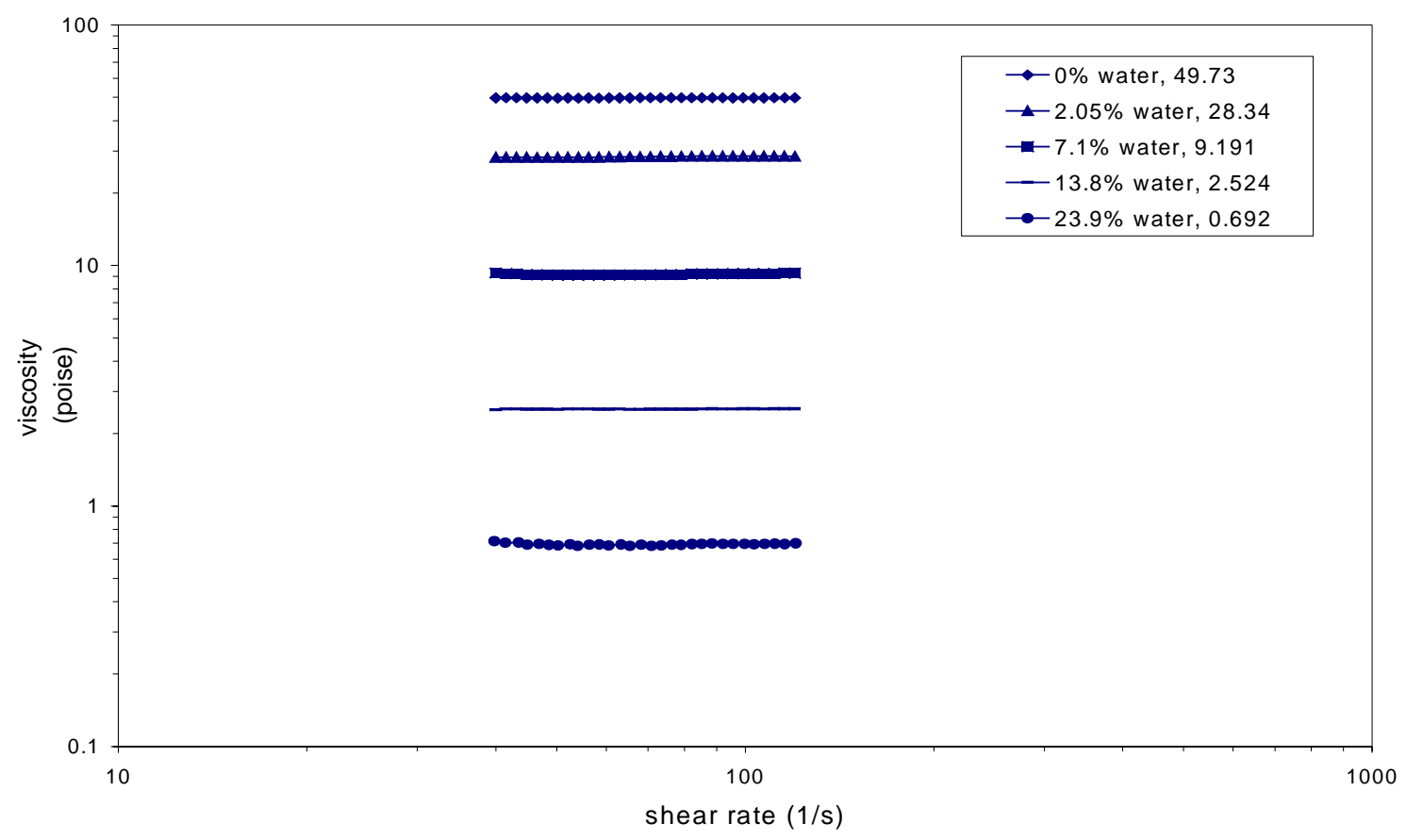

Figure 4.1 Viscosity data for corn syrup-water solutions at $25^{\circ} \mathrm{C}$

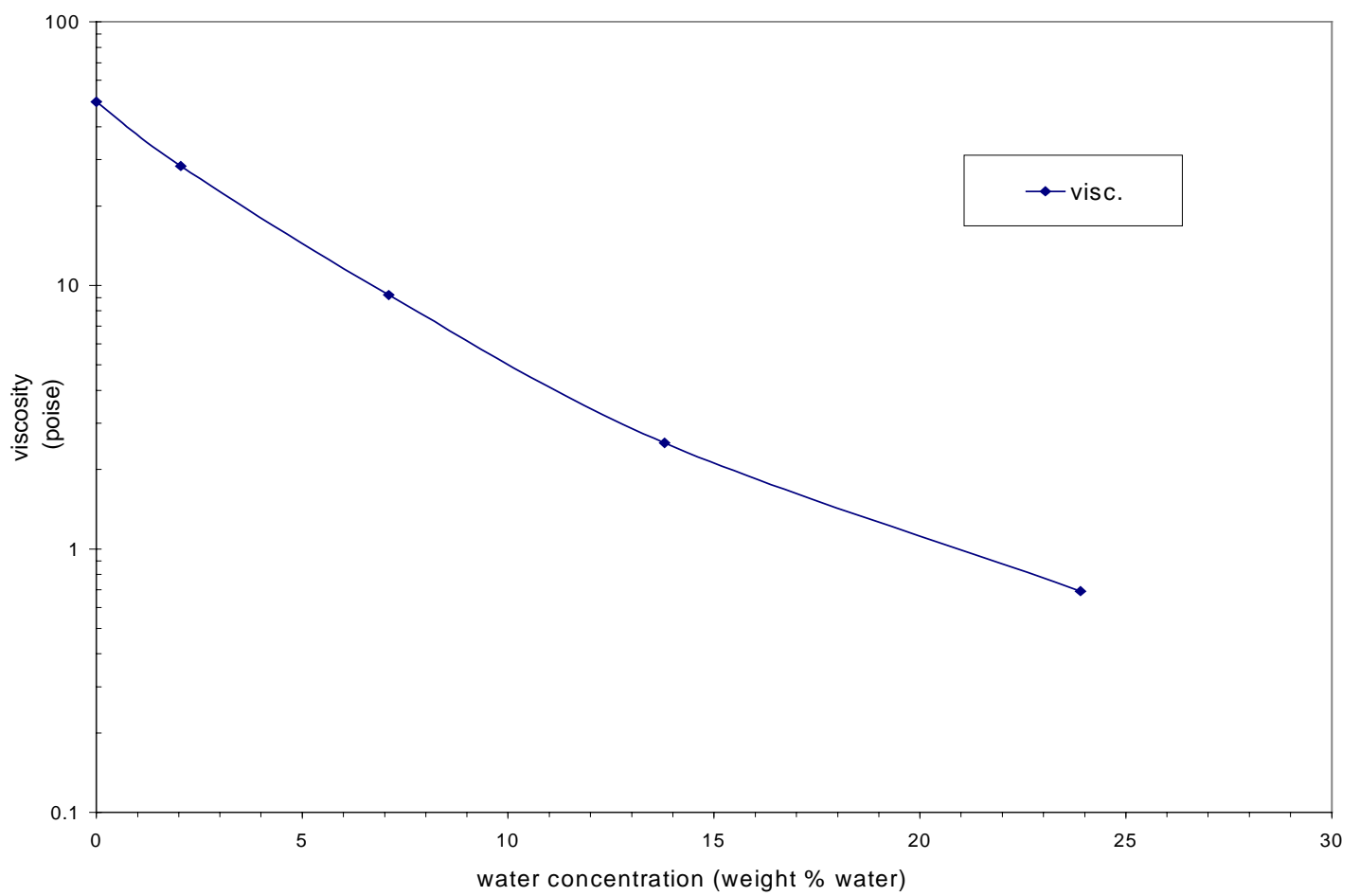

Figure 4.2 Viscosity of corn syrup with different water contents 


\subsection{Steady shear viscosity of fibrid suspensions}

Before measurements could be made on fibrid suspensions, the water content of the as-supplied fibrids was determined. This was done by taking a known amount of each kind of fibrid, drying the sample to remove all the moisture and reweighing the dry sample. Results of this procedure are given in Table 4.2.

The water content of the fibrid samples was taken into account in calculating the polymer concentration in the fibrid suspensions and also in estimating the suspending liquid viscosity. Even so, very inconsistent suspension viscosity results were obtained when the effect of polymer concentration or suspending liquid viscosity was determined by experiment. It was then decided to make a single concentrated fibrid suspension for each fibrid type. These master solutions were diluted by addition of more corn syrup and water to get the desired fibrid concentration and suspending liquid viscosity; details are given in Table B1. In this manner, we eliminated effects associated with the batchto-batch variation in polymer mixing and dispersion. This led to consistent results. Ultimately, we chose three different suspending liquid viscosities for each polymer type. This resulted in a total of 27 different suspensions on which shear viscosity measurements were made. This was a manageable amount of work, and it revealed the influence of fibrid type, fibrid concentration and medium viscosity.

In making viscosity measurement on suspensions, it is necessary that the result not depend on fixture geometery. For parallel plate fixtures, this means that the viscosity should be independent of the gap spacing. It is, therefore, essential that the gap spacing be significantly larger than the largest dimension of the suspended solids. Since the fibrids were expected to be about $100 \mu \mathrm{m}$ in length and width, we attempted to make measurement with gap spacings of $1000 \mu \mathrm{m}$ and $2000 \mu \mathrm{m}$. Unfortunately, we could not work with the larger gap spacing because the liquid flowed out of the gap. This was especially true for the lower viscosity suspensions. Gaps of $1000 \mu \mathrm{m}$ and $1500 \mu \mathrm{m}$ were then used, and results were found to be the same. This is demonstrated in Figure 4.3 for a 0.18 wt\% suspension of F20W in corn syrup containing 23.9 wt\% water. All other experiments were carried out at a gap spacing of $1000 \mu \mathrm{m}$. 
TABLE 4.2 DETERMINING THE ACTUAL POLYMER CONTENT IN MOIST POLYMER SAMPLES

\begin{tabular}{|c|c|c|c|}
\hline Fibrid type & $\begin{array}{c}\text { Weight before } \\
\text { drying }(\mathrm{g})\end{array}$ & $\begin{array}{c}\text { Weight after } \\
\text { drying }(\mathrm{g})\end{array}$ & $\begin{array}{c}\text { Polymer percent } \\
(\%)\end{array}$ \\
\hline F10W & 151.072 & 22.364 & 14.804 \\
\hline F20W & 147.850 & 29.690 & 20.081 \\
\hline F25W & 149.013 & 33.826 & 22.700 \\
\hline
\end{tabular}



Figure 4.3 Gap spacing effect for $0.18 \%$ F20W in corn syrup solution ( $23.9 \mathrm{wt} \%$ water) 
Some additional precautions were taken to obtain reliable and accurate data. Samples were allowed to rest for about a week to allow time for any entrained air bubbles to escape. This was acceptable because there were no sample aging effects. Also, a low viscosity vegetable oil (Wesson) was used to coat the outer rim of the parallel plate fixtures to eliminate problems related to the drying of corn syrup over the course of the experiment. Note that viscosity measurements made on the 27 suspensions were repeated at least once and the same results were obtained.

Figure 4.4 shows the effect of fibrid concentration and fibrid type on the steady shear viscosity of fibrids suspended in a Newtonian medium of 2.5 poise shear viscosity; the accessible range of shear rates is 40 to $1201 / \mathrm{s}$. Several features are distinguishable from the data. The suspension viscosity can be very significantly larger than the suspending medium viscosity, and it decreases with increasing shear rate. Thus, in the case of the F20W fibrid, the addition of only a tiny amount of polymer can increase the viscosity of the liquid by more than an order of magnitude, especially at low shear rates. Increasing the fibrid concentration, at a fixed shear rate, results in a progressive increase in the viscosity in each case. Surprisingly, though, the F20W fibrid suspensions had the highest viscosity level followed by F25W and then F10W. We had expected the F10W suspensions to have the highest viscosity based on their largest aspect ratio. This fact had been observed by DuPont researchers also, and its explanation was one of the objectives of the present research.

The fact that suspensions of F10W fibrids always have the lowest viscosity is further demonstrated in Figures 4.5 \& 4.6. Here the fibrid concentration is kept fixed at either $0.54 \%$ or $0.8 \%$ but the medium viscosity is changed; results at the $0.18 \mathrm{wt} \%$ level were similar. For each medium, the suspension viscosity changes according to $\mathrm{F} 2 \mathrm{WW}>\mathrm{F} 25 \mathrm{~W}>\mathrm{F} 10 \mathrm{~W}$. Indeed, it was not even necessary to make viscosity measurements to come to this conclusion. This was evident simply by observing the fluidity of the three different types of suspensions by inverting the bottles containing these suspensions.

To get a complete picture of the effect of fibrid type, fibrid concentration and medium viscosity, it is necessary to examine data over a wider shear rate range. This was done by Dr. R.F. Liang in our research group, and his findings are summarized 
here (Liang et al., 2001). These data were obtained with the RMS 800 viscometer at the same temperature but for shear rates between $10^{-4}$ and $400 \mathrm{~s}^{-1}$.

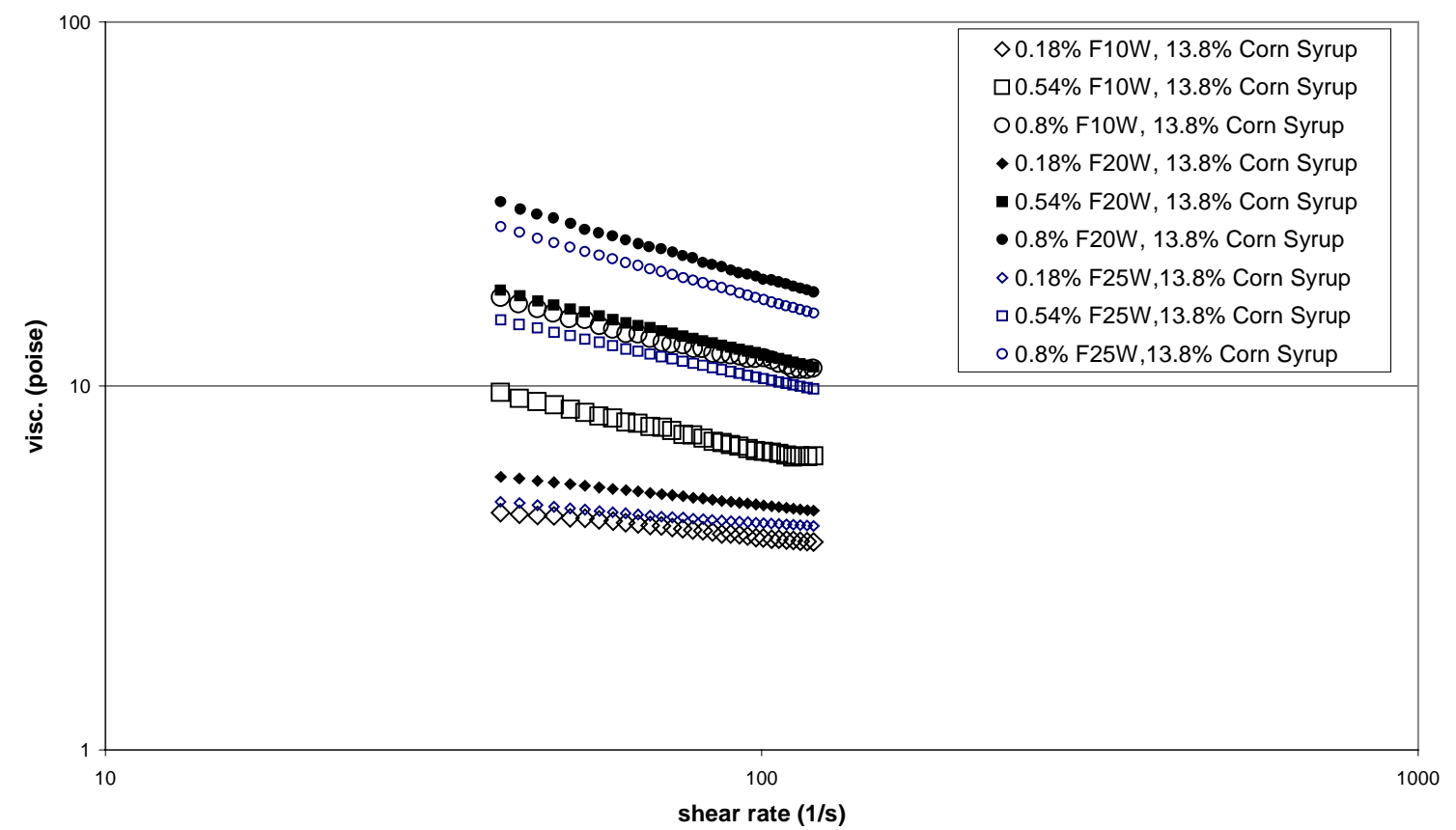

Figure 4.4 Effect of solid loading for fibrids in matrix fluid (13.8\% water in corn syrup)

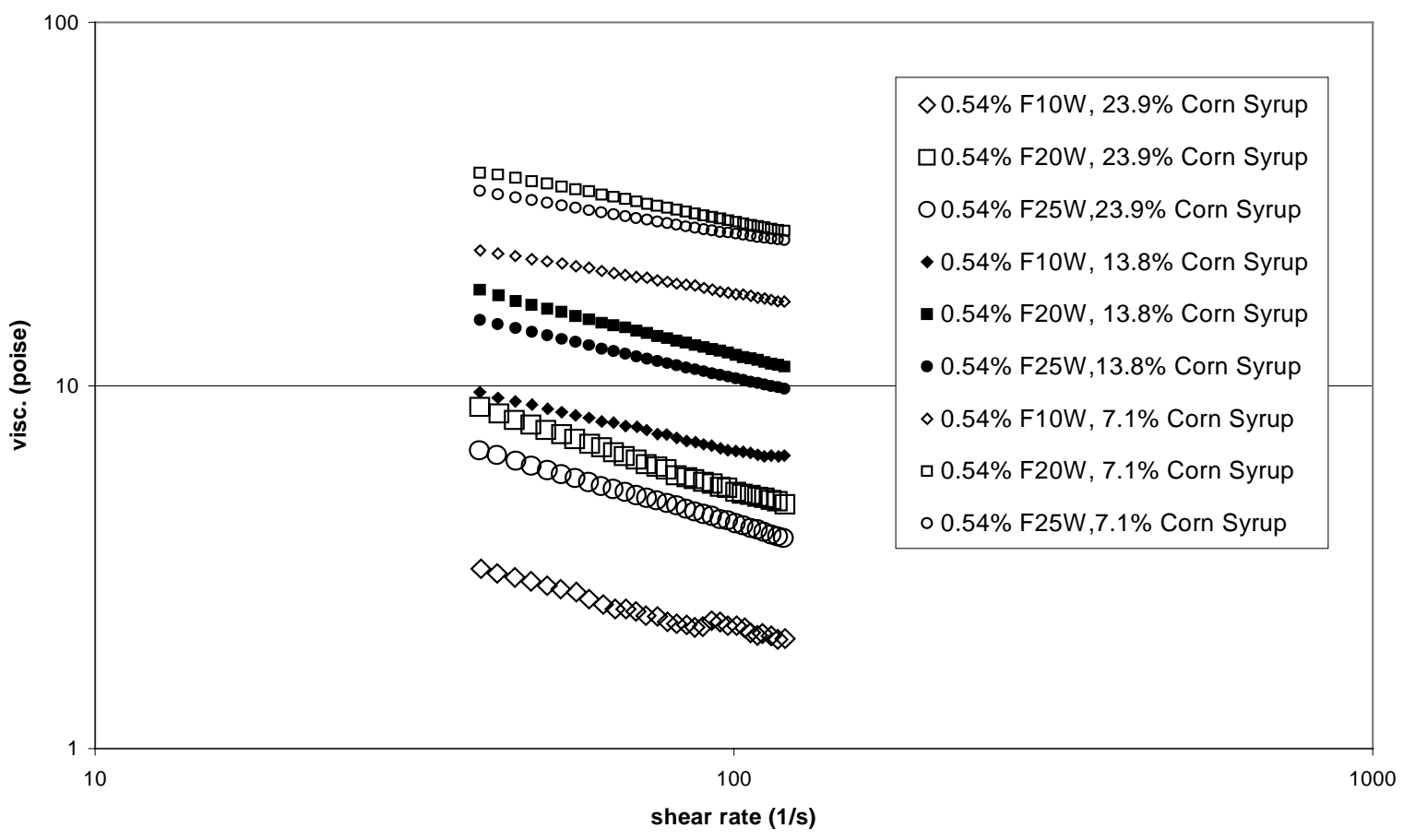

Figure 4.5 Effect of fibrid size for $0.54 \%$ fibrid 


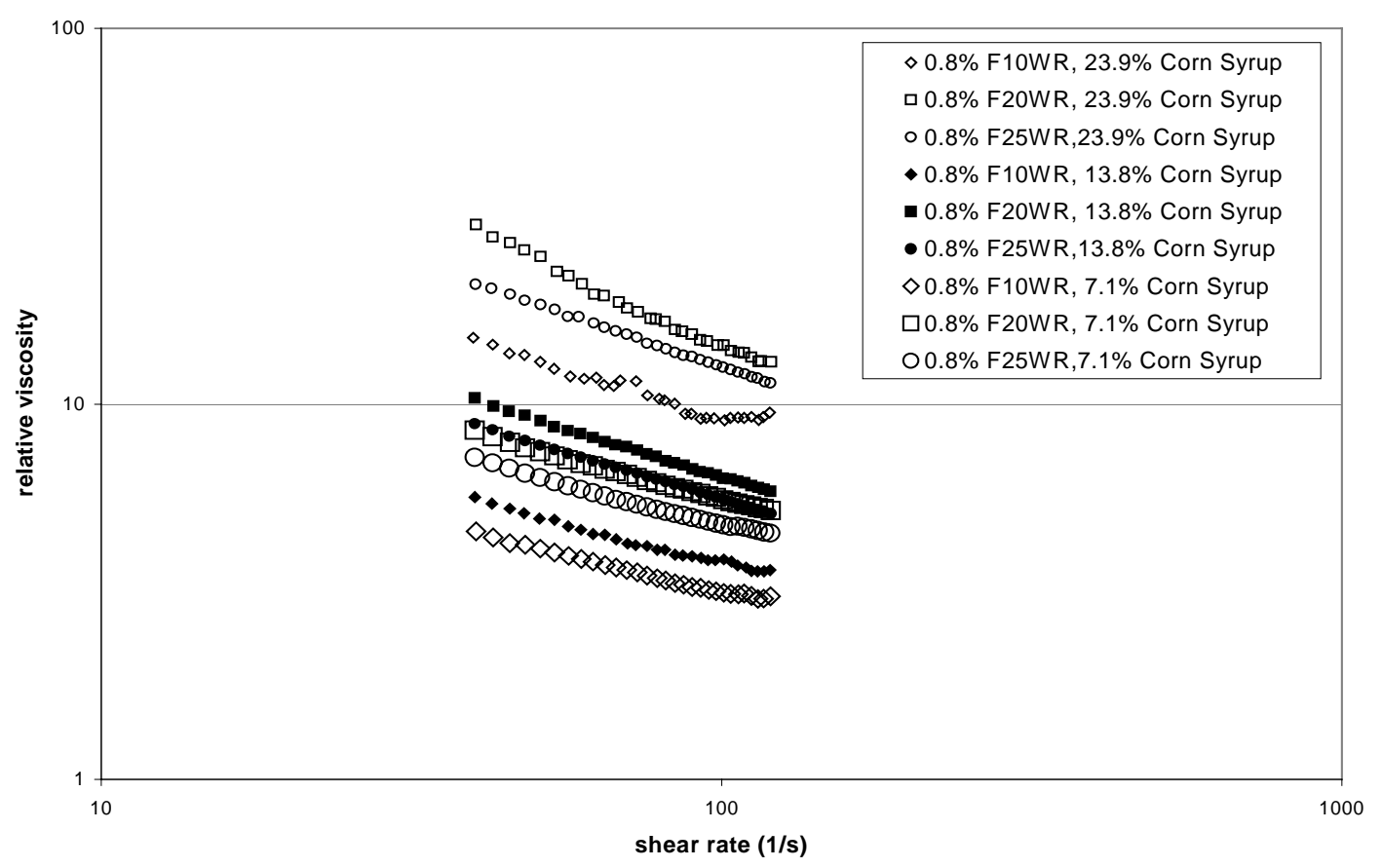

Figure 4.6 Effect of Matrix Fluid Viscosity for $0.8 \%$ fibrids

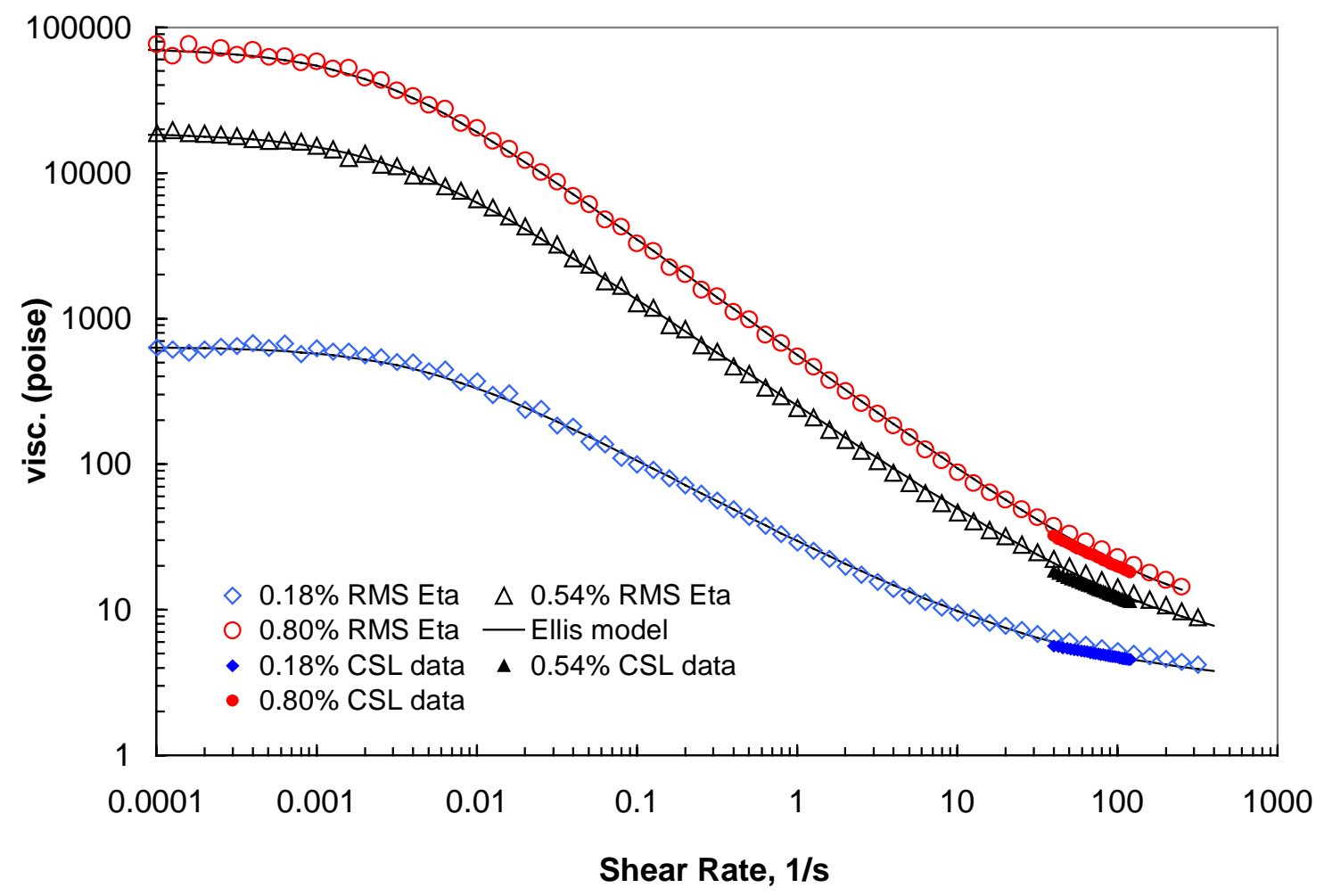

Figure 4.7 Effect of solid loading for F20W in corn syrup solution (13.8\% water) 
Figure 4.7 displays the effect of fibrid concentration of the F20W suspension in $0.245 \mathrm{~Pa} \cdot \mathrm{s}$ corn syrup solution as a function of shear rate. Also shown on this figure are the CSL 100 results which were limited to a shear rate range between 40 and 120 $\mathrm{s}^{-1}$. It is seen that agreement between the RMS 800 and CSL 100 results is very good. This figure shows that fibrid suspensions have a constant viscosity plateau at low shear rates followed by a power-law region with increasing shear rates, and finally, there is an approach to another constant viscosity region at very high shear rates. At the high shear rate end, all the curves tend to approach the suspending medium viscosity, but the higher concentration suspension always has the higher viscosity.

The effect of fibrid type /structure on the steady shear viscosity of $0.54 \%$ fibrid suspensions in 0.245 Pa.s corn syrup is illustrated in Figure 4.8. All three types of fibrids with different aspect ratio were considered. Using the medium viscosity as a reference, a tremendous viscosity enhancement with a more than three decade increase in viscosity is seen at low shear rates for all three suspensions due to adding $0.54 \%$ fibrids. The F20W suspension has the highest viscosity over the entire shear rate range followed by the $\mathrm{F} 25 \mathrm{~W}$ suspension, while the $\mathrm{F} 10 \mathrm{~W}$ suspension has the lowest viscosity. As is also seen from this figure, the three suspensions have parallel shear thinning behavior, indicating a power law index independent of fibrid structure. But at high shear rates the three curves appear to converge to the same infinite shear viscosity.

Figure 4.9 illustrates the effect of medium viscosity on shear viscosity of suspensions of $0.54 \% \mathrm{~F} 20 \mathrm{~W}$ fibrids in three corn syrup/water solutions as a function of shear rate. It can be concluded from Figure 4.9 that the zero-shear viscosities of the suspensions of all fibrid types are effectively independent of the suspending medium viscosity and determined only by the morphology and concentration of the fibrids. The matrix viscosity only affects the flow behavior in the shear thinning region and at very high shear rates. These results indicate that the equilibrium microstructure at low shear rates is governed only by the unique morphology and adjustable concentration of the fibrids; at high shear rates the microstructure is disrupted because of the higher stresses imparted. 


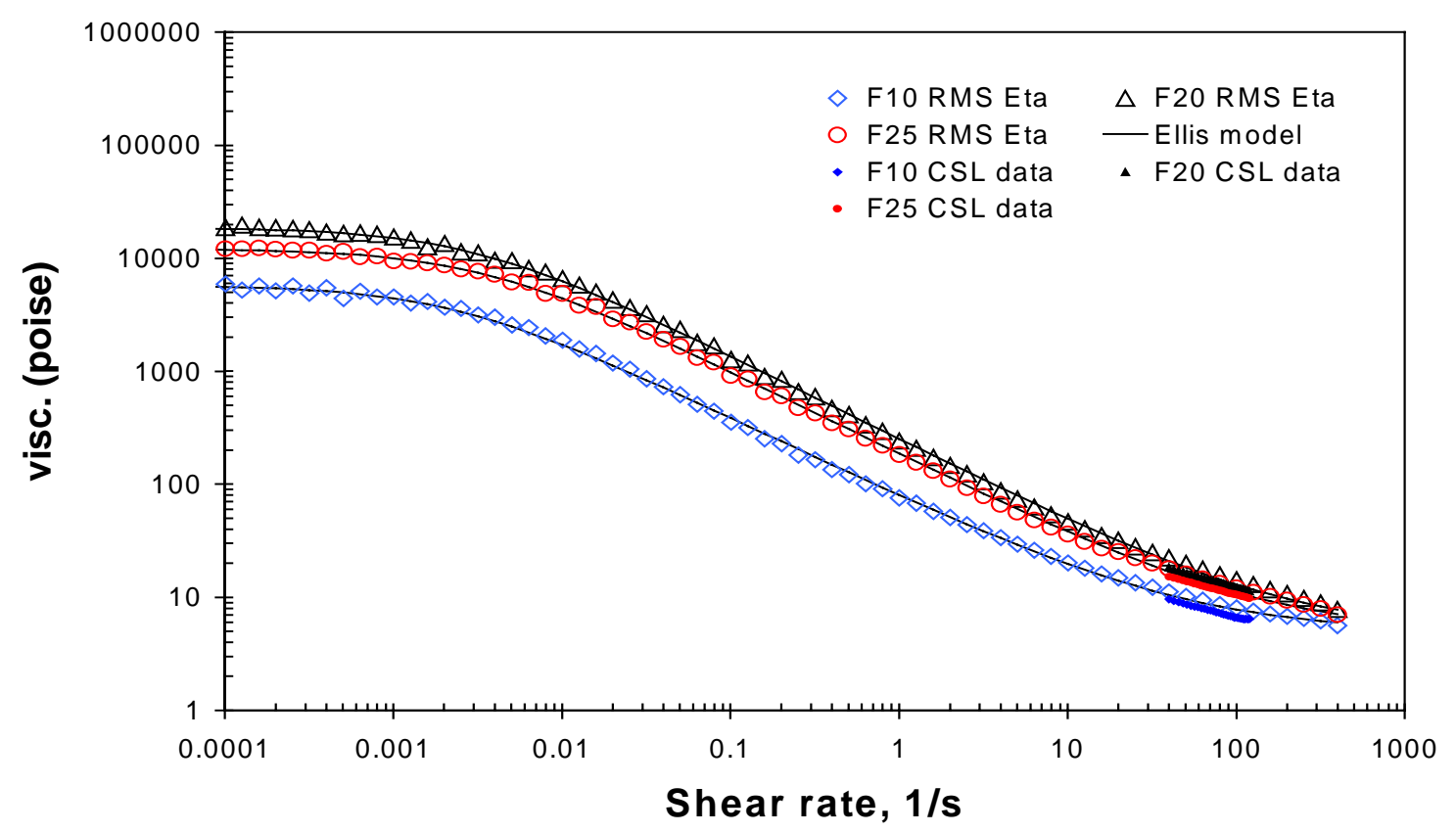

Figure 4.8 Effect of fibrid type for $0.54 \%$ fibrids in corn syrup solution ( $13.8 \%$ water)

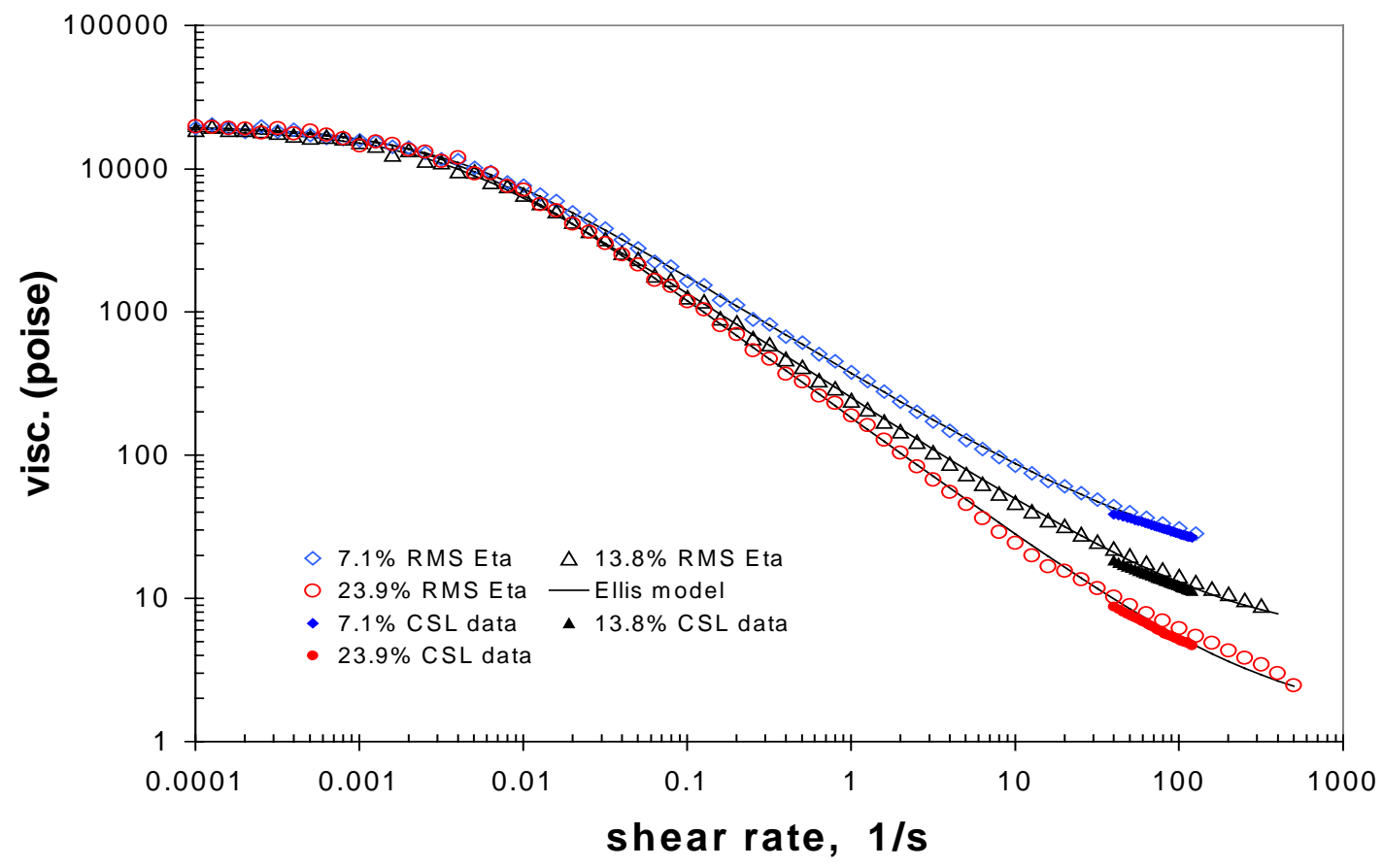

Figure 4.9 Effect of medium viscosity for $0.54 \%$ F20W in corn syrup solution 
Based on all of the foregoing, it appears that the fibrids associate with each other via entanglement formation, and the strength of this network (rather than the medium viscosity) determines the suspension viscosity at low shear rates. Also that the F20W fibrids give the strongest network and the F10W fibrids give the weakest network. With increasing shear rate, the network is broken down, and this shows up as shear thinning with the viscosity following a power-law behavior. At very high shear rates, the network is completely destroyed, and the suspension viscosity approaches the medium viscosity; at a given polymer concentration, the suspension viscosity becomes independent of fibrid type, but increasing polymer concentration results in an enhancement in the viscosity.

In terms of data representation, each of equations $2.6-2.8$ was found to do an equally good job of fitting the data over the entire shear rate range, and the method of estimating the model parameters is described in Liang et al. (2001). Representative fits to a single data set are shown in Figure 4.10. This says that equations traditionally employed to describe the viscosity behavior of particulate suspensions are just as useful for fitting data on platelet suspensions.

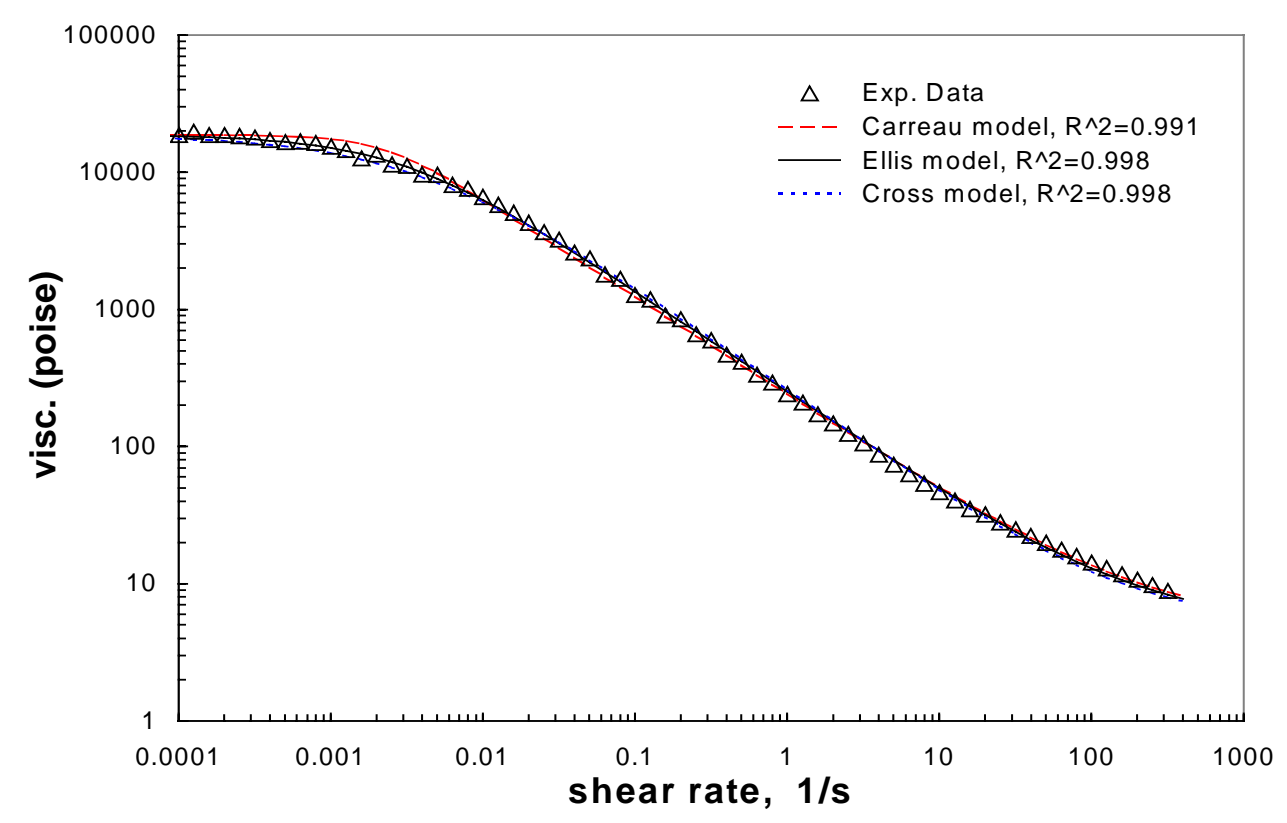

Figure 4.10 Comparison of model fits with experimental data for $0.54 \% \mathrm{~F} 20 \mathrm{~W}$ suspension in corn syrup solution (13.8\% water) 


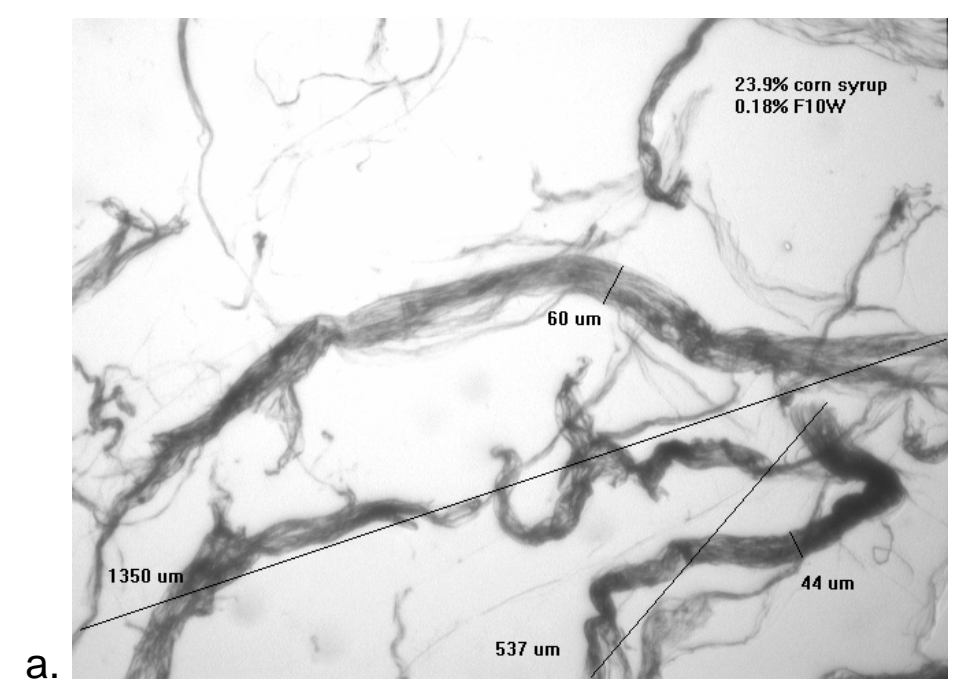

b.

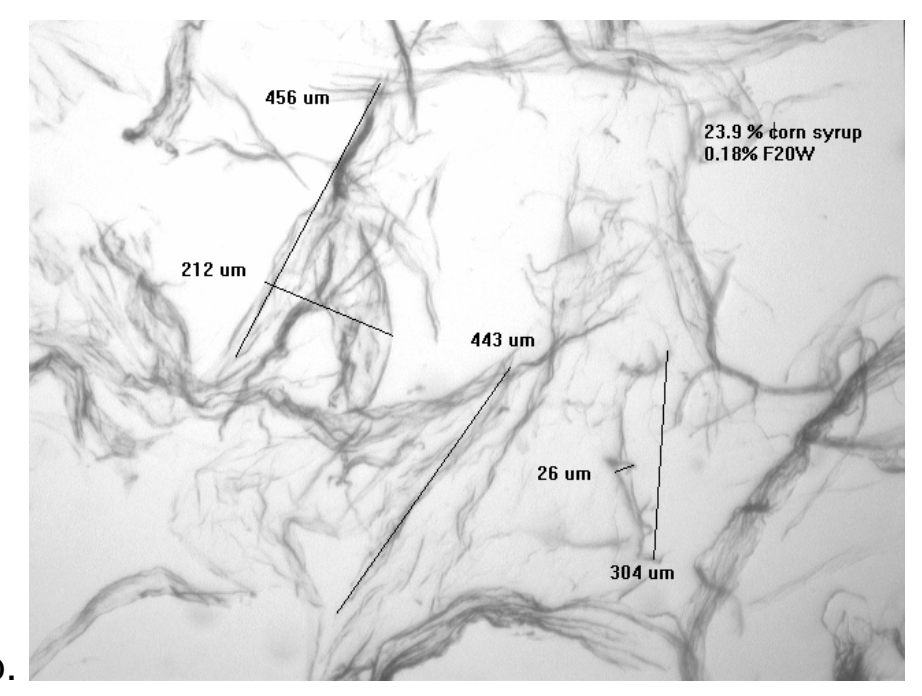

C.

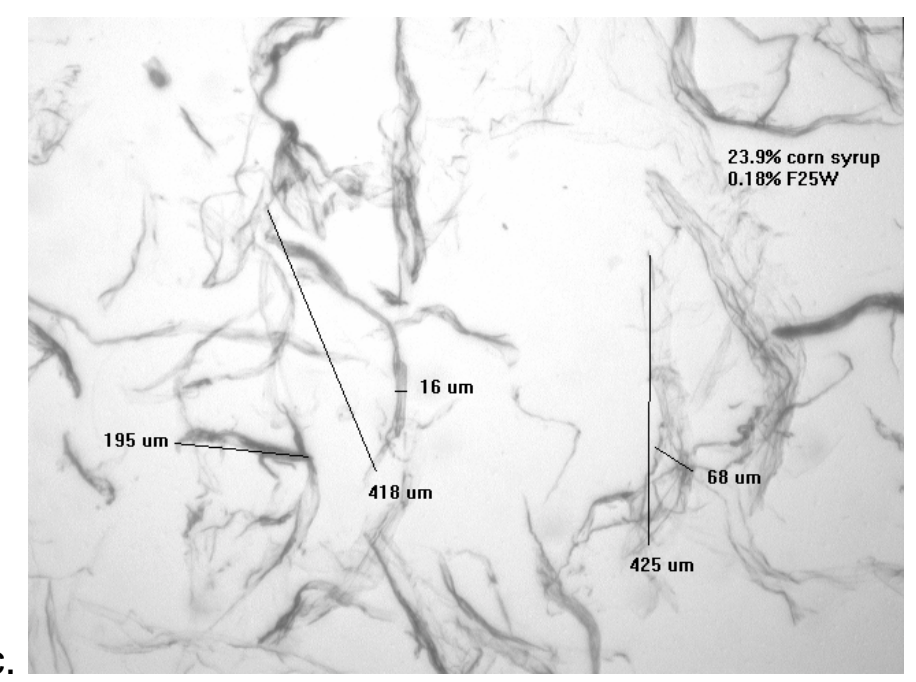

Figure 4.11 Typical images of $0.18 \%$ fibrids dispersed in corn syrup solution $(23.9 \%$ water); a: F10W, b: F20W; c: F25W 
Finally, to shed some light on why F10W suspensions had such a low viscosity, photographs were taken of the individual samples by placing them below a microscope before shearing, and these are shown in Figure 4.11.

We see from the images that the F10W suspension is composed of a cluster of large amount of individual fibrids and its largest dimension is over $1 \mathrm{~mm}$. In contrast to this, F20W and F25W suspensions are better dispersed, and we can even observe individual fibrids and there are not many noticeable differences between F20W and F25W. Although F10W has the largest individual dimension among the three grades of fibrids, F10W has the least "space-filling" network, and this leads to F10W's lowest viscosity at the same loading level as compared as F20W and F25W.

From these images, we conclude that F10W is noticeably different from F20W and F25W and its cluster size is much larger than that of the other two fibrids. The difference in dispersion situation is the reason for the observed shear viscosity trends.

Figures B1 - B18 give detailed results for the shear measurement, and Table B1 gives the details about preparation of suspensions. 


\subsection{Einstein coefficient of fibrid suspensions}

When a filler is added to a Newtonian liquid, its viscosity increases in a manner given by the Einstein equation,

$$
\eta_{s}=\eta_{m}\left(1+K_{E} \phi\right)
$$

where $\eta_{\mathrm{s}}$ and $\eta_{\mathrm{m}}$ are the suspension and matrix viscosities respectively, $\phi$ is filler volume fraction and $K_{E}$ is a constant whose value depends on filler shape and orientation; for spheres, $K_{E}$ equals 2.5 . The ratio $\eta_{s} / \eta_{m}$ is called the relative viscosity $\eta_{R}$, and it is generally measured as a function of $\phi$ using a glass capillary viscometer of the type shown in Figure 3.3. One keeps the viscometer vertical, fills the lower bulb with the solvent and allows the liquid to drain through the capillary under the influence of gravity. One notes the drainage time $t_{m}$. The experiment is repeated with the suspension, and the drainage time $t_{s}$ is recorded. The relative viscosity is then given by

$$
\eta_{R}=\frac{t_{s}}{t_{m}}
$$

Knowing $\eta_{R}$ as a function of $\phi$, the Einstein coefficient $K_{E}$ is obtained as

$$
K_{E}=\operatorname{Lim}_{\phi \rightarrow 0}\left(\frac{\eta_{s}-\eta_{m}}{\eta_{m} \phi}\right)
$$

The Einstein coefficient typically increase as the degree to which the streamlines are disturbed by the particle increases. Note, though, that the Einstein equation is valid only for the infinite dilution suspensions where there are no particle-particle interactions. The purpose of carrying out measurements on infinite dilution fibrid suspensions using a glass capillary viscometer was to examine how the Einstein coefficient might reflect the effect of fibrid structure on viscosity enhancement.

Before any measurements on ppm concentration fibrid suspensions were made, the experimental method was evaluated for its reliability using glass bead suspensions. The different glass bead having a density of $2.56 \mathrm{~g} / \mathrm{cc}$ and average diameter of $4 \mu \mathrm{m}$ and $11 \mu \mathrm{m}$ were used as were hollow glass bead of density $1.128 \mathrm{~g} / \mathrm{cc}$ and an average diameter of $1.1 \mu \mathrm{m}$. Four different matrix liquids were used. They were hydraulic oil, corn syrup (commercial grade), diluted corn syrup 1 (add 18\% weight water in corn 
syrup) and diluted corn syrup 2 (add 20\% weight water in corn syrup). Their properties are listed in Table 4.3. All the experiments were done at $25^{\circ} \mathrm{C}$.

TABLE 4.3 COMMON PROPERTIES OF MATRIX SOLVENT

\begin{tabular}{|c|c|c|c|c|}
\hline & $\begin{array}{c}\text { Hydraulic } \\
\text { Oil }\end{array}$ & $\begin{array}{c}\text { Corn } \\
\text { Syrup }\end{array}$ & $\begin{array}{c}\text { Diluted corn } \\
\text { syrup 1 (18\% } \\
\text { water) }\end{array}$ & $\begin{array}{c}\text { Diluted corn } \\
\text { syrup 2 }(20 \% \\
\text { water })\end{array}$ \\
\hline $\begin{array}{c}\text { Density } \\
\left(\mathrm{g} / \mathrm{cm}^{3}\right)\end{array}$ & 0.881 & 1.382 & 1.313 & 1.306 \\
\hline $\begin{array}{c}\text { Viscosity } \\
\text { (poise) }\end{array}$ & 1.046 & 41.62 & 1.612 & 1.156 \\
\hline
\end{tabular}

When experiments were done with solid glass beads dispersed in corn syrup, inconsistent results were obtained, and these are described in Appendix C. Indeed, unrepeatable results were obtained regardless of the diameter of the beads, whether 4 $\mu \mathrm{m}$ or $11 \mu \mathrm{m}$. It was then decided to employ a hydraulic oil as the suspending medium, and the quality of the data improved markedly. Typical results of both kinds of solid glass beads are displayed in Figure 4.12. However, the slope of the straight line plot of relative viscosity versus volume fraction turned out to be approximately 2 instead of the anticipated value of 2.5. This, however, is due to sedimentation resulting from the density difference between the glass beads and the oil. In order to substantiate this conclusion, hollow glass beads of $1.1 \mu \mathrm{m}$ diameter were suspended in the same hydraulic oil and the experiments repeated. Since the density difference was now greatly reduced, relative viscosity data (shown in figure 4.13) yielded the correct Einstein coefficient of 2.5 .

Next, relative viscosity measurements were made on the hollow glass beads/corn syrup system, and results are presented in Figure 4.14. Surprisingly, the Einstein coefficient turned out to be 6.76 . To see if density mismatch was again to blame for the error, the corn syrup was diluted with water to lower its density. This procedure 
reduced the viscosity also. Hollow glass beads were dispersed in corn syrup containing $18 \%$ water and also in corn syrup containing $20 \%$ water. Relative viscosity data in these two suspending media are shown in Figure 4.15 and 4.16, and the correct Einstein coefficient is obtained. It is for this reason that dilute corn syrup was used when making measurement with Nomex ${ }^{\circledR}$ fibrids. These results are presented next.

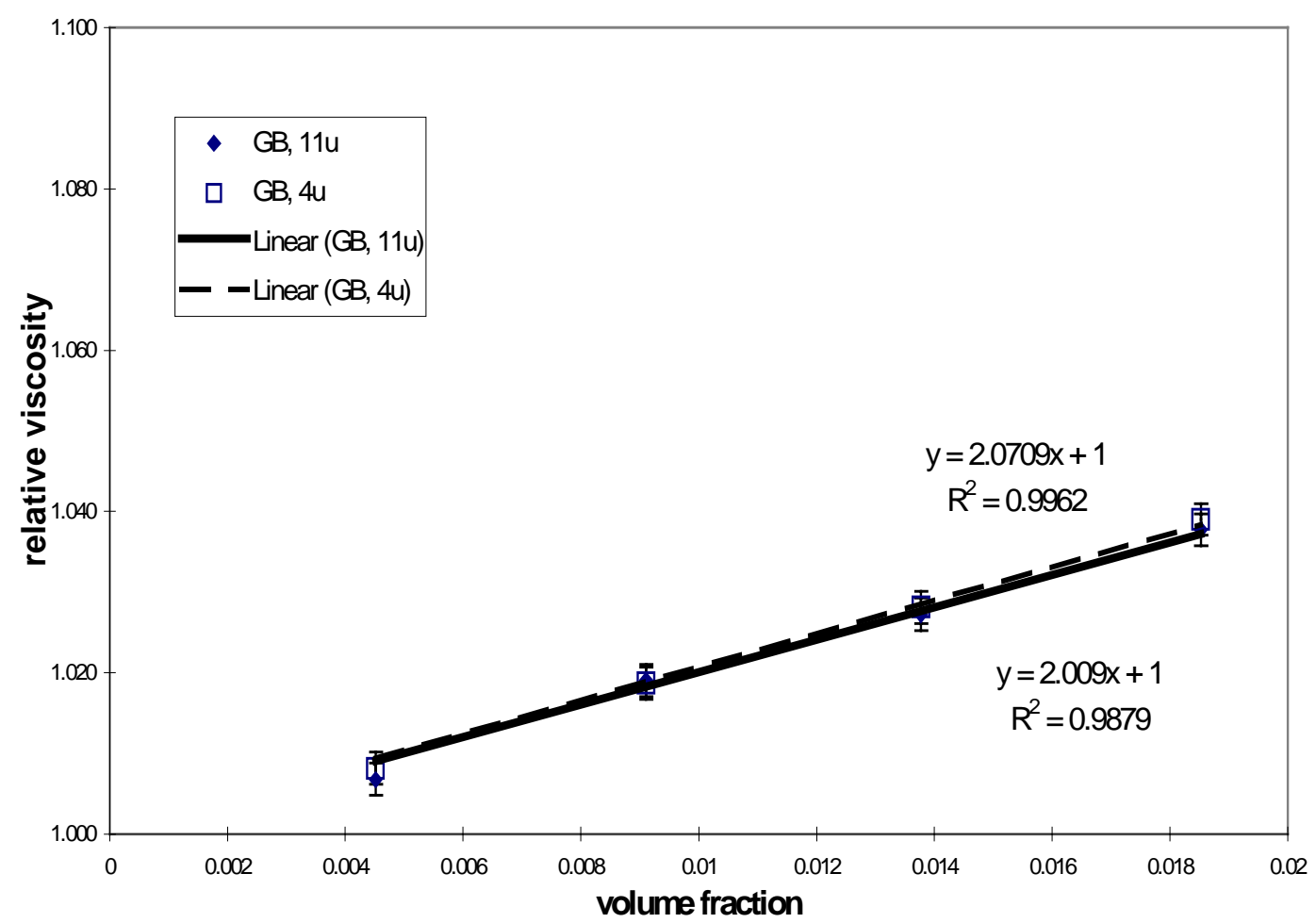

Figure 4.12 Determination of Einstein Constant for glass beads in hydraulic oil suspension 


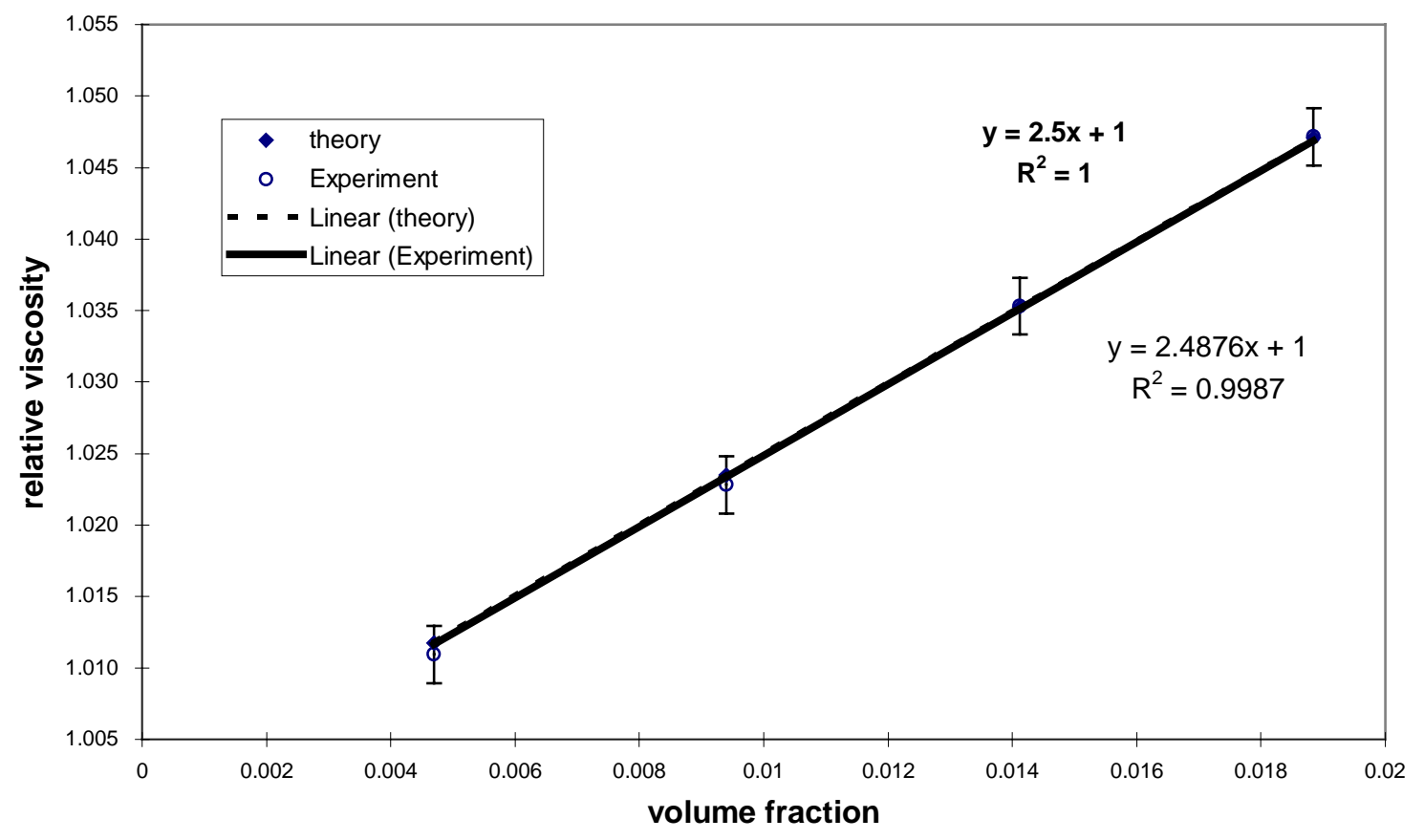

Figure 4.13 Determination of Einstein Constant for hollow glass bead in hydraulic oil suspension

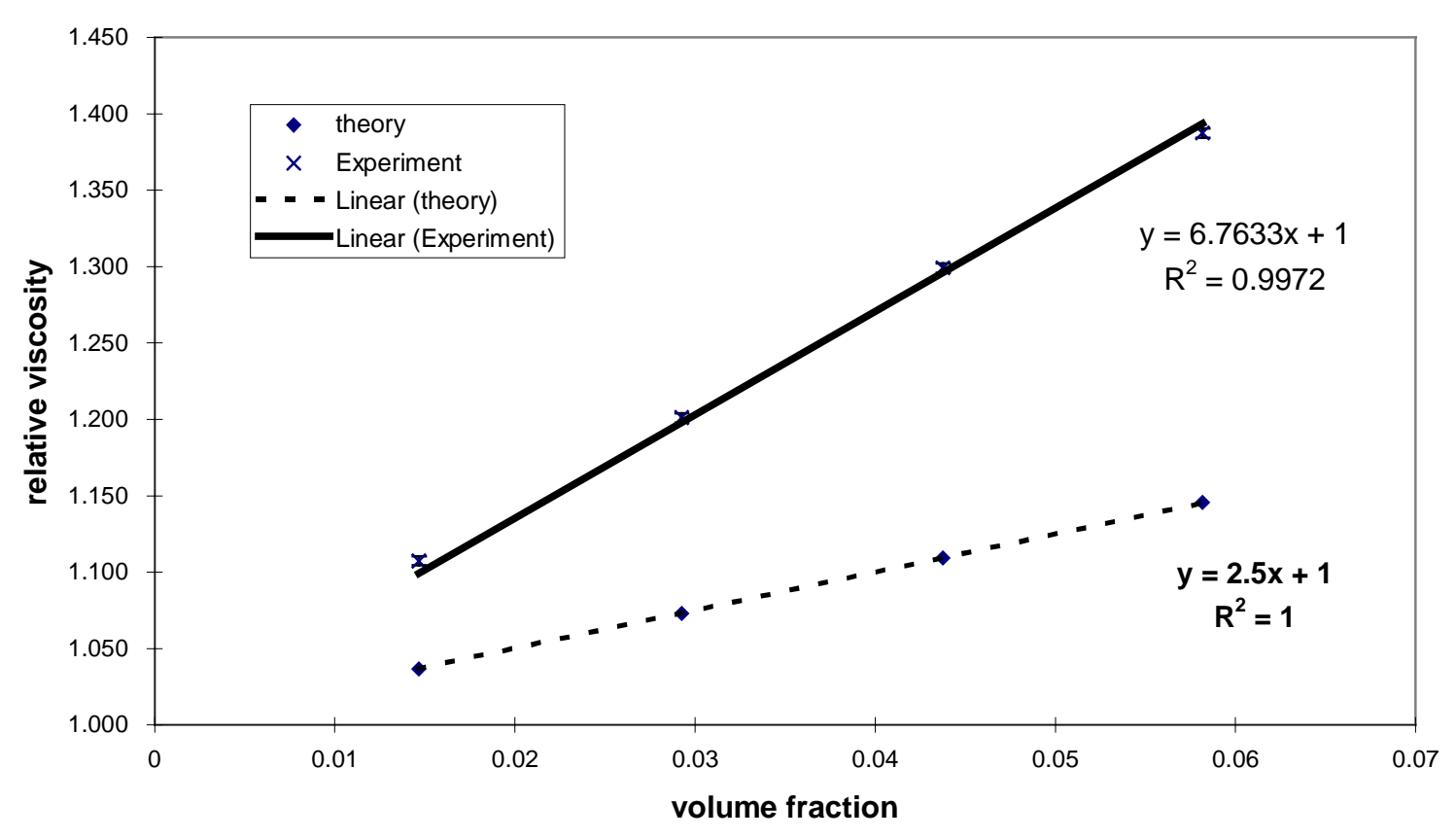

Figure 4.14 Determination of Einstein Constant for hollow glass bead in corn syrup suspension 


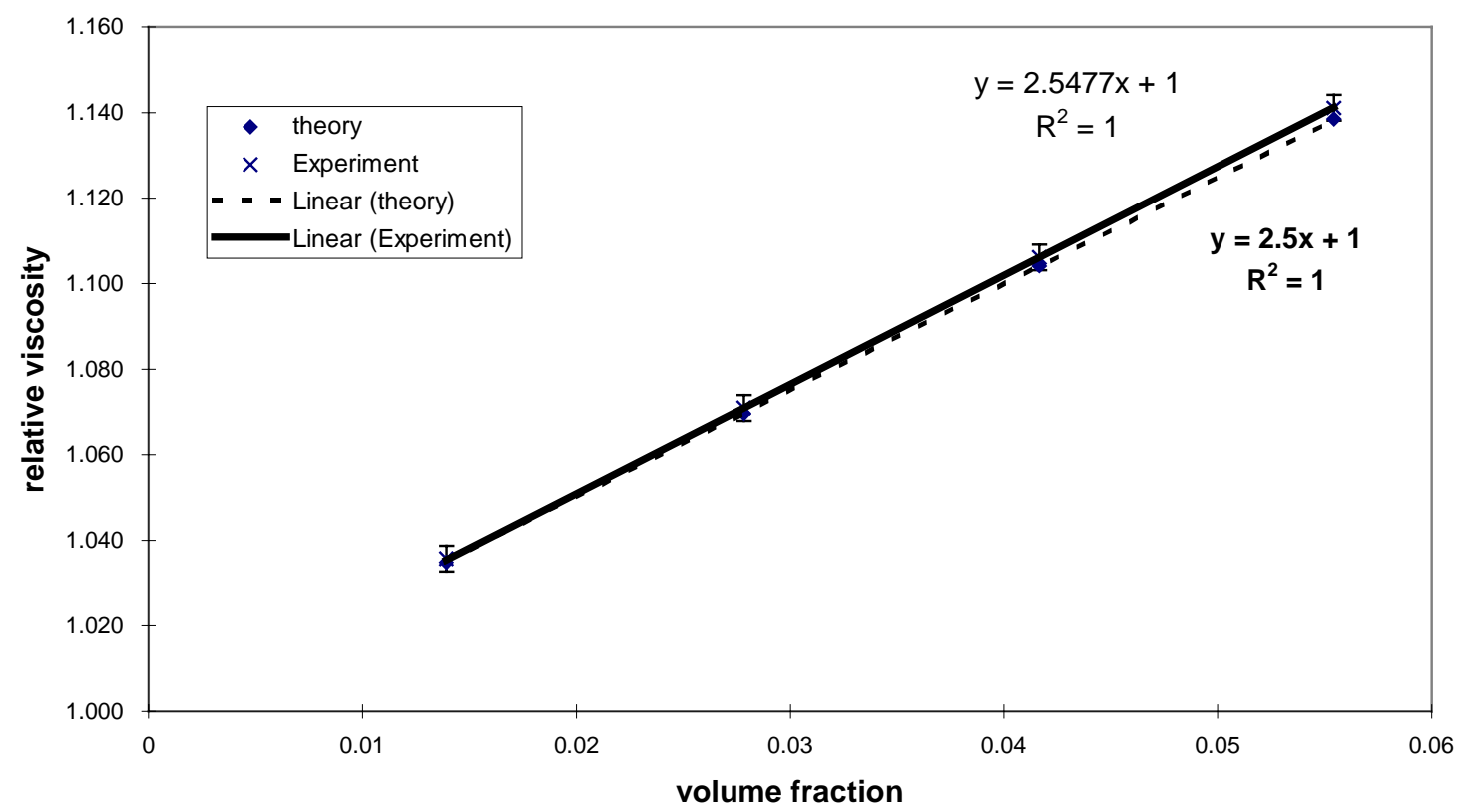

Figure 4.15 Determination of Einstein Constant for hollow glass bead in corn syrup 1 (18\% water) suspension

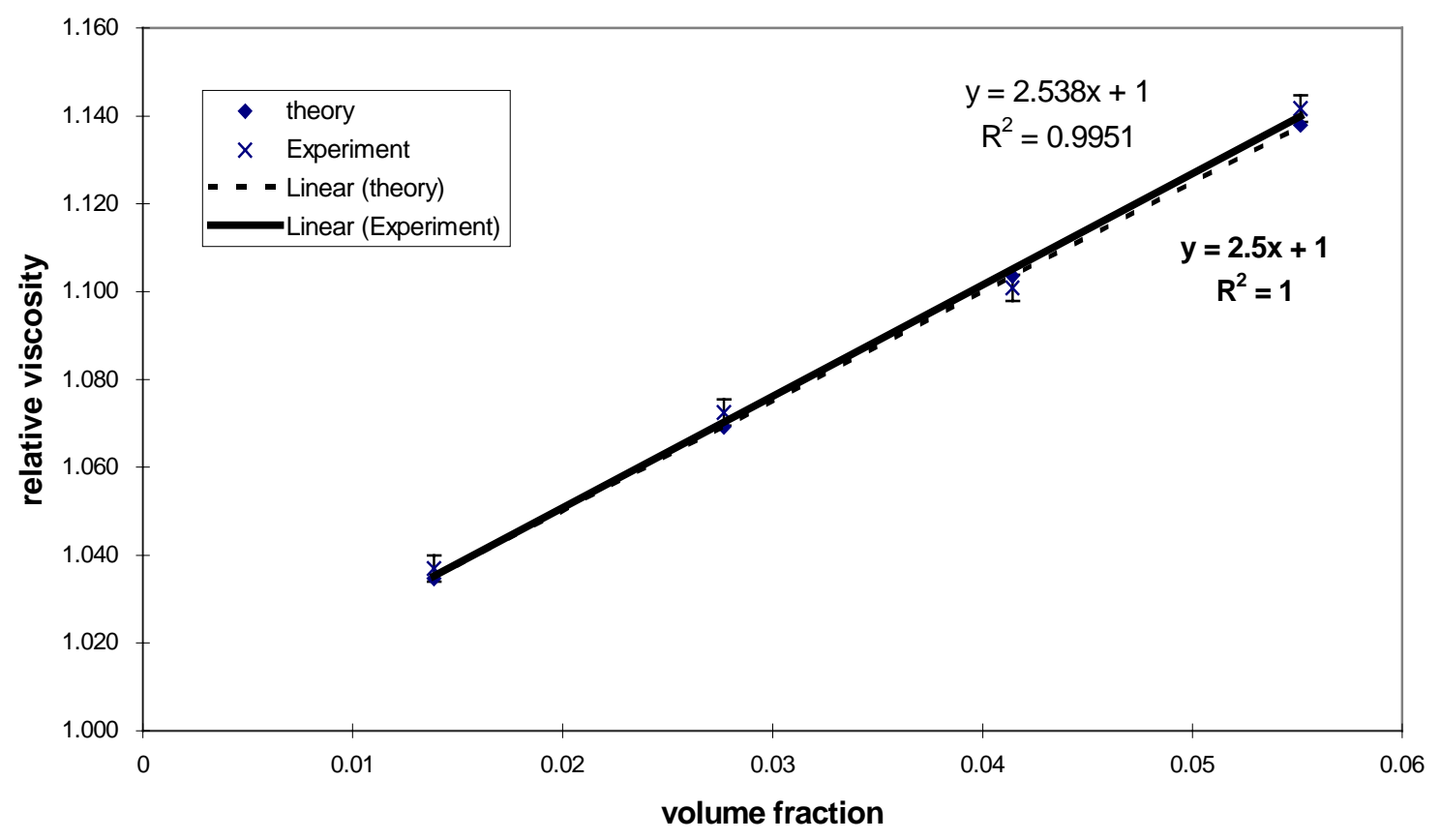

Figure 4.16 Determination of Einstein Constant for hollow glass bead in corn syrup 2 (20\% water) suspension 
When relative viscosity data on the fibrid suspensions were plotted against the volume fraction, slightly non-linear plots were obtained regardless of whether dilute corn syrup 1 or dilute corn syrup 2 was the suspending liquid. Linear plots were, however, obtained when the experimental procedure was changed to ensure that exactly the same amount of liquid was charged to the viscometer in each run. Figure 4.17 and 4.18 show the final results. It is seen that the Einstein coefficient for the fibrid suspensions are extremely large, and the largest value corresponds to F10W fibrids. Thus, in this flow field and at low concentrations, the inherently larger fibrid exhibits the largest flow resistance, and the smallest fibrid exhibits the smallest flow resistance.

The above results indicate the viscosity enhancement effect of different types of fibrids, in other words, the "space-filling" ability at infinite dilution, following the order of $\mathrm{F} 10 \mathrm{~W}>\mathrm{F} 20 \mathrm{~W}>\mathrm{F} 25 \mathrm{~W}$. These trends are consistent with what we expected from the aspect ratio (L/D) trends of the fibrids specified by DuPont. This result indicates that the dispersion of $\mathrm{F} 10 \mathrm{~W}$ fibrids at the ppm level had been improved in comparison with the state of dispersion in $\sim 1 \%$ concentration level. The fact that fibrids have Einstein coefficients over 150 times larger than $K_{E}=2.5$ for spheres reflects the remarkable space-filling nature of fibrids and helps demonstrate their tremendous viscosity enhancement effect. If we consider fibrids as uniaxially oriented fibers, whose Einstein coefficient is $2 \mathrm{~L} / \mathrm{D}$, then the Einstein coefficient would be at least 2000 (if we choose $L / D$ is 1000). This figure is much larger than the experiment results.

To conclude this section, we present photographs in Figure 4.19 of dilute suspensions containing 50 ppm of each type of fibrid in corn syrup 1 (18\% weight water).

We find from the images that $\mathrm{F} 10 \mathrm{~W}$ is dispersed much better than F10W suspension which was used in shear response measurement (refer to figure 4.11). The cluster of fibers in figure 4.11 has now changed into a "web" like fibril network. There are few noticeable differences between the three grades of fibrid suspensions. It seems that the "space-filling" network leads to the remarkable viscosity enhancement of a fibrid suspension.

Table $\mathrm{C} 1-\mathrm{C} 13$ give some further details about the preparation of suspensions and measured results. 


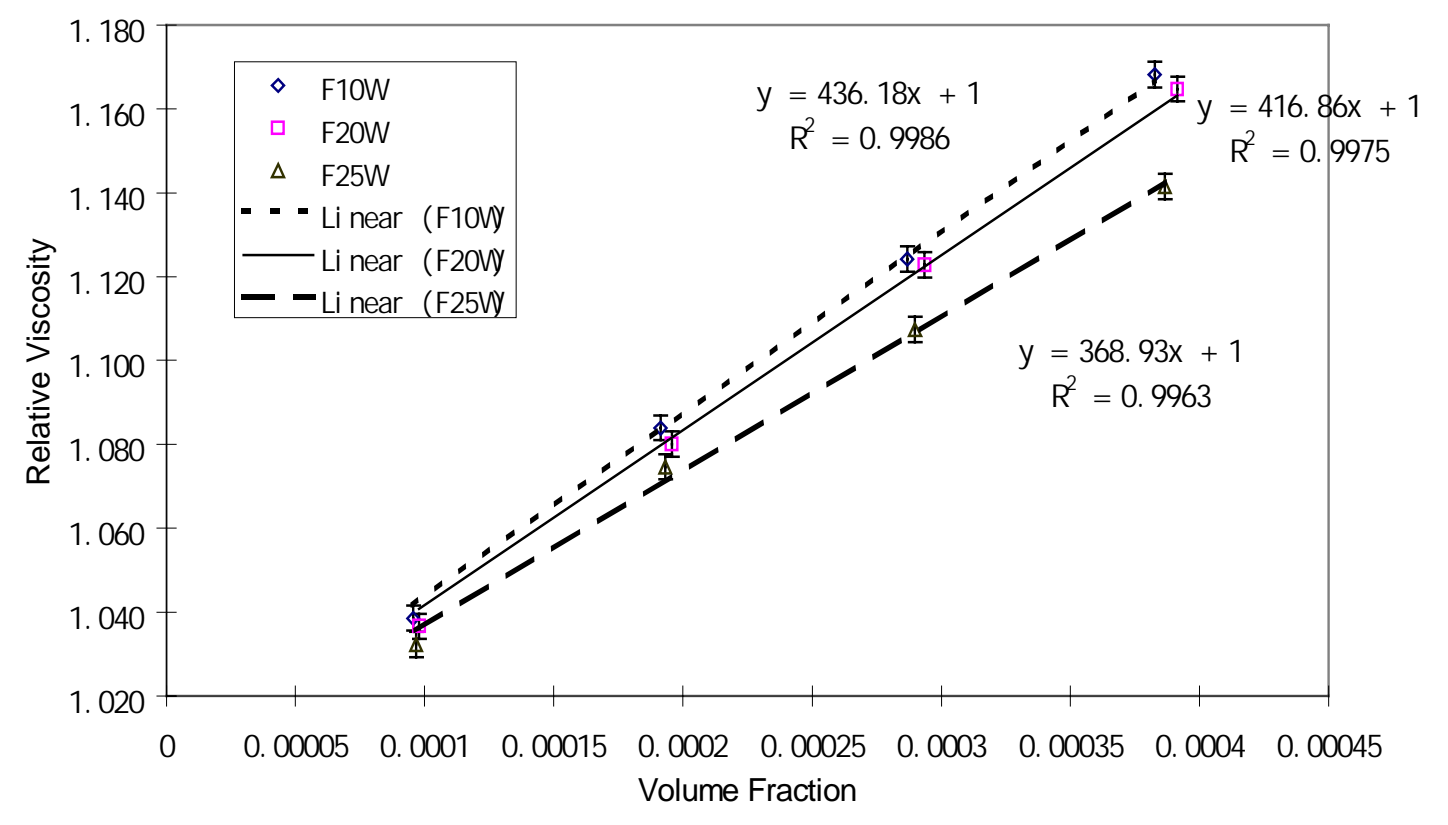

Figure 4.17 Determination of Einstein Constant for fibrids in corn syrup 2 (20\% water) suspension (Final results)

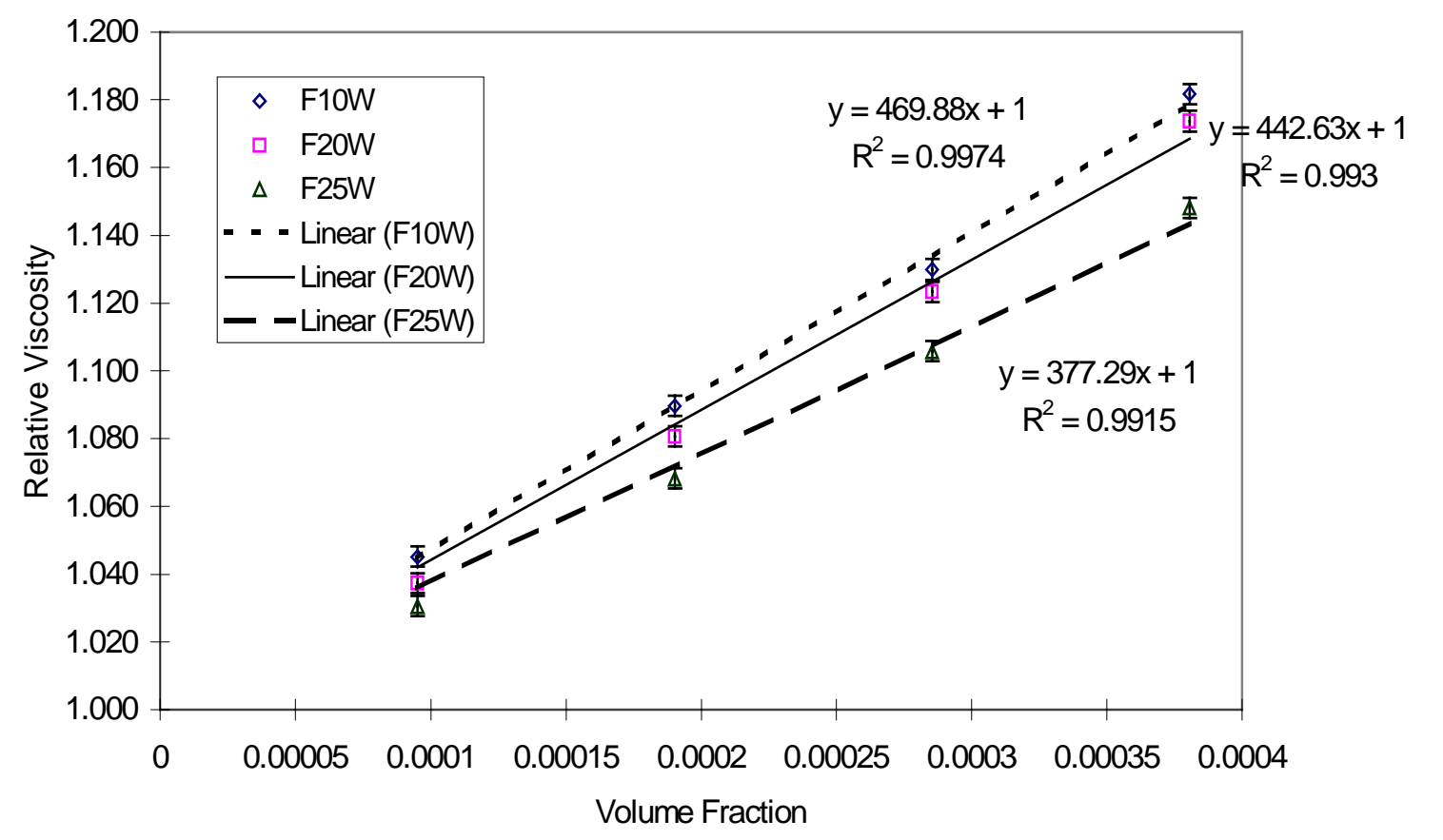

Figure 4.18 Determination of Einstein Constant for fibrids in corn syrup 1 (18\% water) suspension (Final results) 

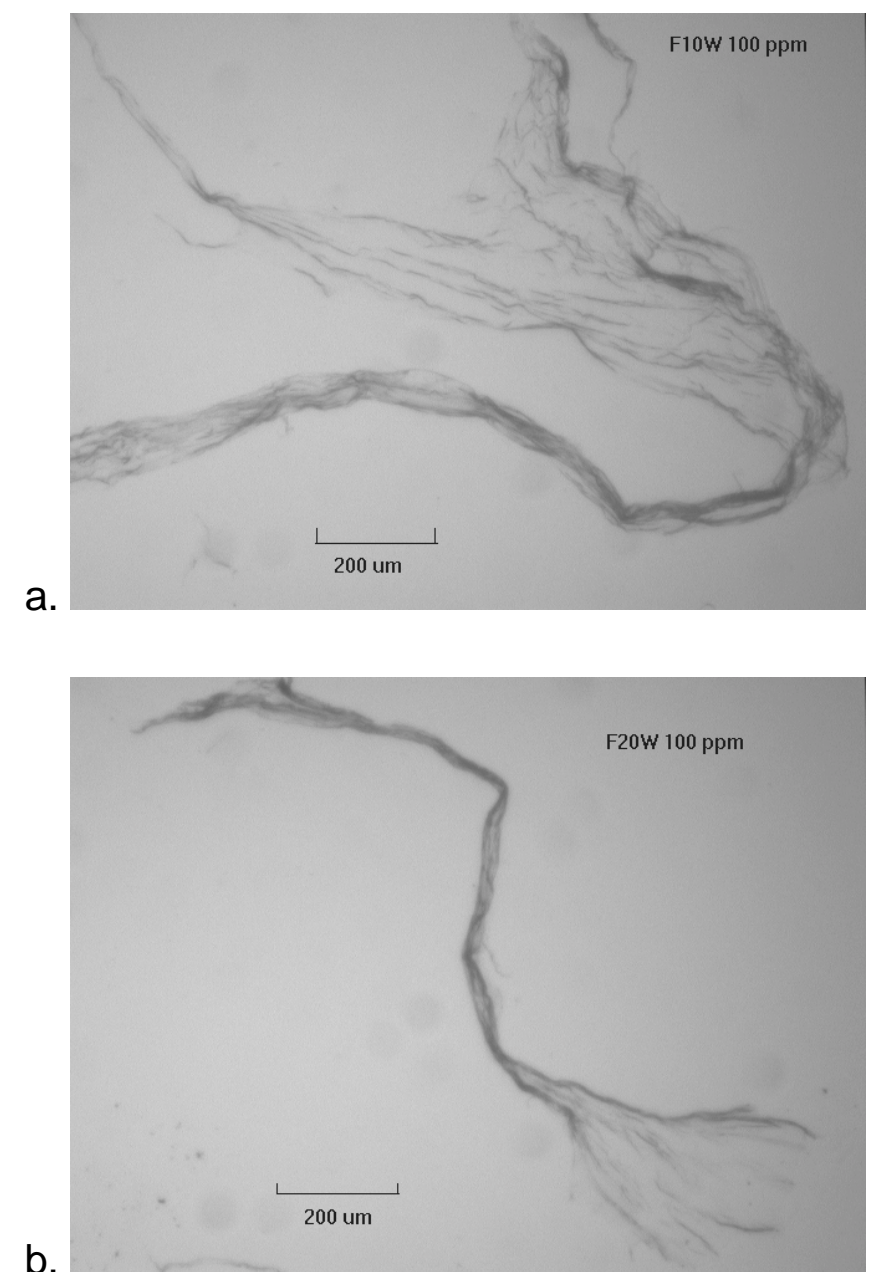

C.

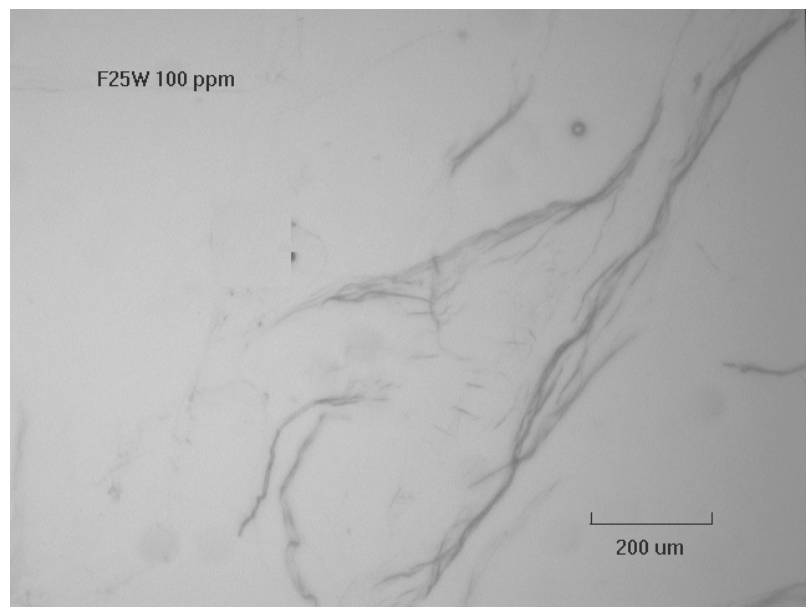

Figure 4.19 Typical images of 100 ppm fibrids dispersed in corn syrup 1 (18\% water); a:F10W; b:F20W; c:F25W 


\subsection{Extensional viscosity measurement}

Shown in Figure 4.20 are photographs of a 200 ppm suspension of F25W in a corn syrup/water mixture of 0.51 poise viscosity ( $25 \mathrm{wt} \%$ water in corn syrup). They are the images at the moment of 'without suction', ' before suction', and ' after suction', respectively. The image ' without suction' shows the moment that suspension exits freely from the upper capillary. The filament diameter at the exit of upper capillary is even larger than the inner diameter of capillary. The image ' before suction' shows the moment that suspension exits freely from the upper capillary and falls to the lower capillary without vacuum suction. Some suspension is not falling down through the inside of lower capillary tube, but spills down along the outside of the lower capillary. The image ' after suction' shows the moment that suspension exits from the upper capillary and falls directly into the lower capillary after applying the vacuum suction. The filament diameter at the inlet of the lower capillary decreases sharply as compared to the other positions, and it is even less than the inner diameter of the lower capillary.

Stretching experiments were done at $25^{\circ} \mathrm{C}$ for each of the three type of fibrid suspensions at a polymer concentration of $200 \mathrm{ppm}$. The tensile stress due to the stretching encountered at the exit of the top capillary was determined from the pressure difference $\left(\mathrm{P}_{\mathrm{b}}-\mathrm{P}_{\mathrm{a}}\right)$. The stretch rate at the exit of the top capillary depends on the flow rate and the distance between the top and bottom capillaries. Also the stretch rate increases as one travels from the top to the bottom capillary. Calculated values of the extensional viscosity as a function of the stretch rate are shown in Figure 4.21. Also shown in this figure are the corresponding shear viscosities which are essentially Newtonian at these low concentrations. Note that the shear viscosity of each of the suspensions is only marginally greater than the suspending medium viscosity. However, the extensional viscosity of the suspension is remarkably higher than that of the dispersion medium. The extensional data of the suspension show a stretch thinning behavior and an extensional viscosity of 14 to 56 times higher than the shear viscosity. It is amazing that adding fibrids at such a low concentration (200ppm) could develop this extent of extensional viscosity enhancement. Two different data sets from two independent extensional measurements at an interval of 10 days demonstrate a very good reproducibility. This is also seen from Figure 4.21 where the suspension of F10W fibrids exhibits the higher extensional viscosity while the F25W suspension shows the 
lowest extensional viscosity. This trend is what would be expected based on the aspect ratio of the individual fibrids.

a.

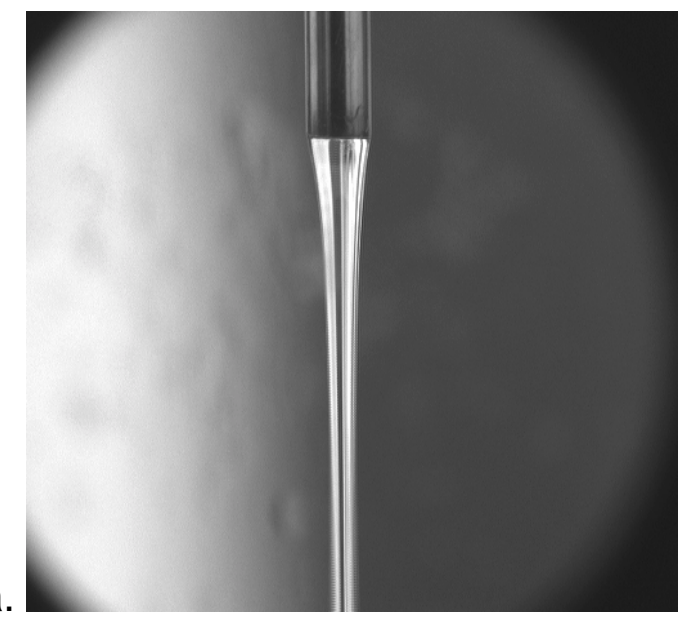

b.

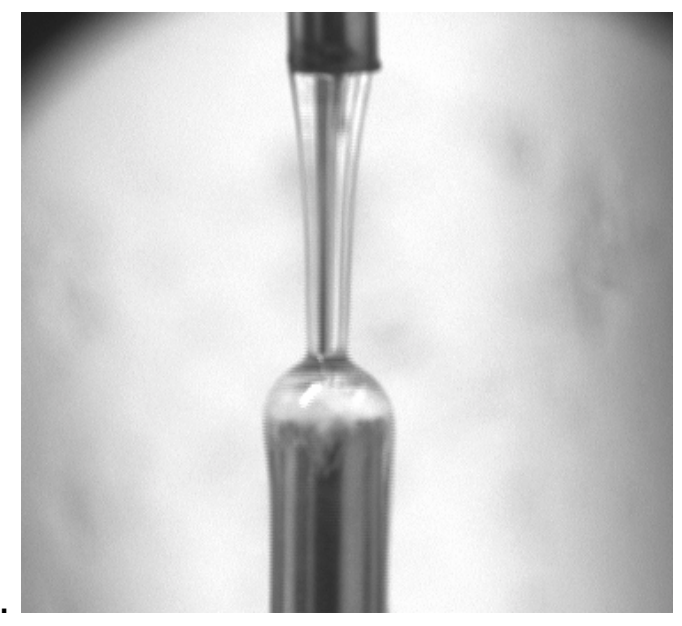

c.

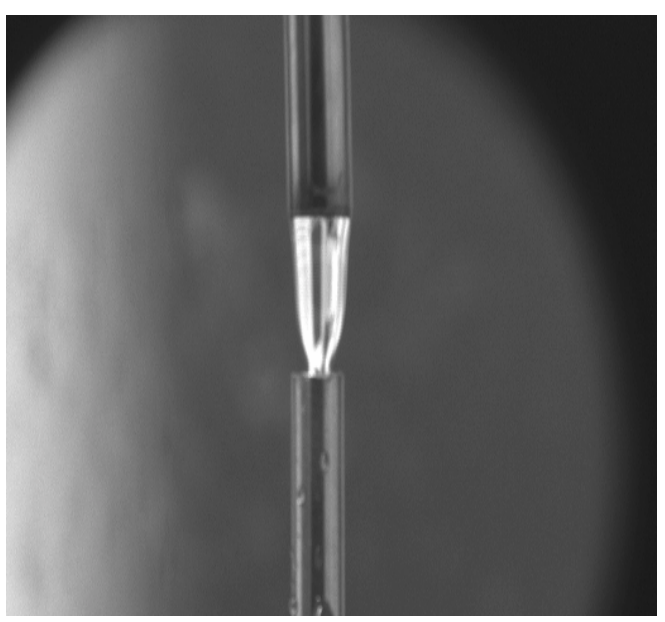

Figure 4.20 Typical image observed during the experiment procedure a. without suction; b. before suction; c. after suction 


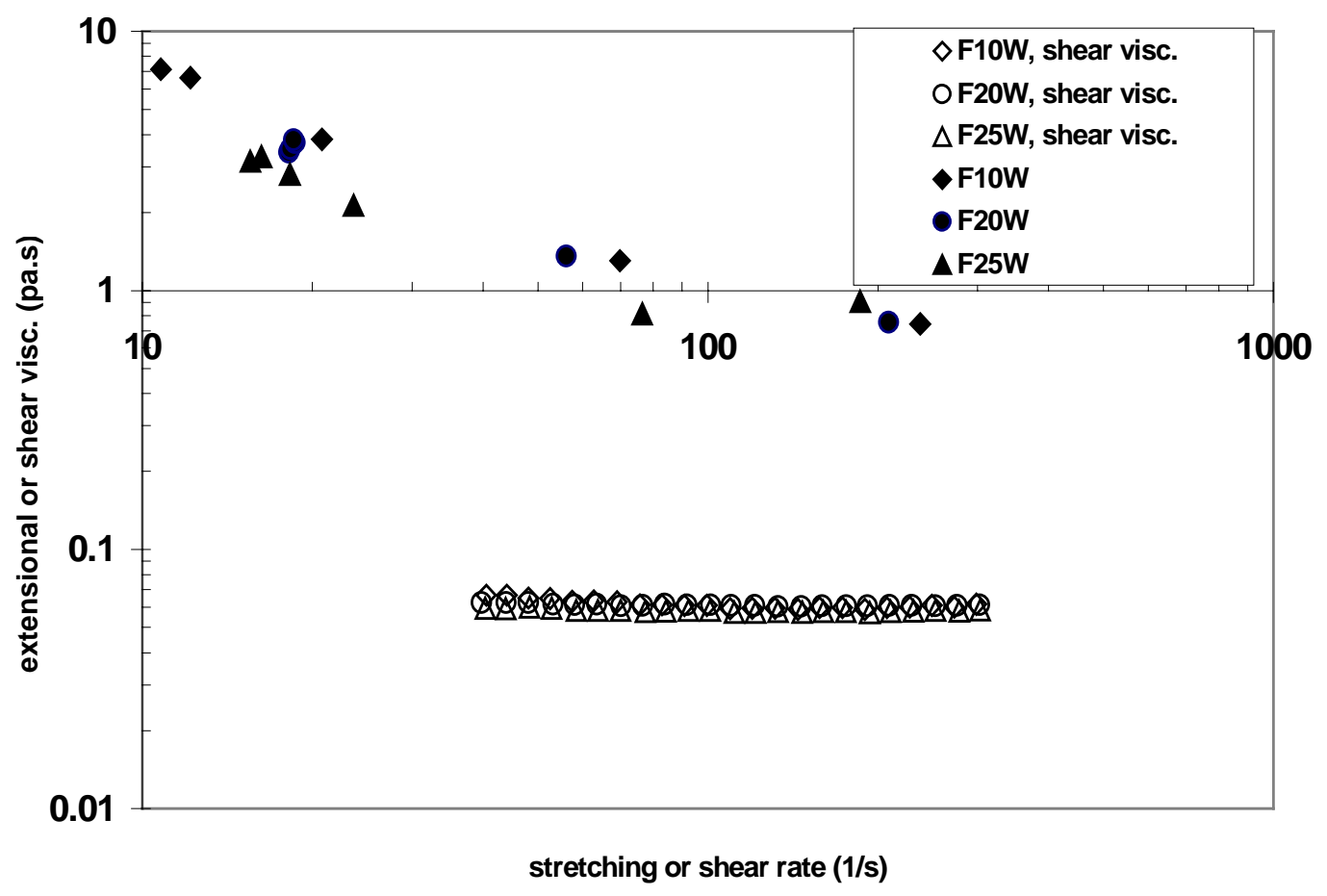

Figure 4.21 Extensional Viscosity for 200ppm Fibrids Suspensions in corn syrup solution ( $25 \%$ water in weight) in Comparison with Shear Viscosity

According to the definition of semiconcentrated region of a fiber suspension (refer to Equation 2.9), $200 \mathrm{ppm}$ fiber suspensions belong to this region. If we assume the aspect ratio (L/D) of comparable fibers to be between 1000 -- 4000, and have the same volume fraction, $\phi$, calculated to be 0.00018 , and suspended in a liquid of viscosity 0.06 Pa.s, then from equation 2.10 (Batchelor, 1971), the relative extensional viscosity or Trouton ratio $\left(\eta_{E} / \eta\right)$ is

$$
\frac{\eta_{E}}{\eta}=\left[3+\frac{4 \phi(L / D)^{2}}{3 \ln (\pi / \phi)}\right]
$$

The results of applying Eq. 2.10 to the three fibrid suspensions are listed in Table 4.4. Remarkably, these results give values similar to those measured and displayed earlier in Figure 4.21. Thus, it seems that the order of magnitude of the extensional viscosity of fibrid suspensions can be predicted from the equations derived for the extensional viscosity of fiber suspensions having the same aspect ratio. 
To conclude this section, Figure 4.22 shows typical images for the three kinds of fibrids after stretch, the fibrid concentration is $200 \mathrm{ppm}$ in $25 \%$ corn syrup solution.

We can see from the images the existence of a network structure. Unlike the fibrid morphology in the solutions used for shear measurements, the microstructure of the stretched fibrids has changed from entangled coils to platelets. This transition increases the degree of space-filling tremendously. It seems that the "space-filling" network accounts for the extensional viscosity enhancement of the fibrid suspensions.

Figure D1 - D6 give some further detail about the measurement results, and Table D1 - D3 give the calculation details for the final results of extensional viscosity.

TABLE 4.4 CALCULATED RESULTS FROM BATCHELOR EQUATION FOR EXTENSIONAL VISCOSITY AND RELATIVE VISCOSITY (TROUTON RATIO)

\begin{tabular}{|c|c|c|c|c|}
\hline Aspect Ratio (L/D) & 1000 & 2000 & 3000 & 4000 \\
\hline $\begin{array}{c}\text { Extensional Viscosity, } \\
\text { (poise) }\end{array}$ & 16.81 & 61.82 & 136.85 & 241.88 \\
\hline Relative Viscosity (-) & 28.0 & 103.0 & 228.1 & 403.1 \\
\hline
\end{tabular}


a.

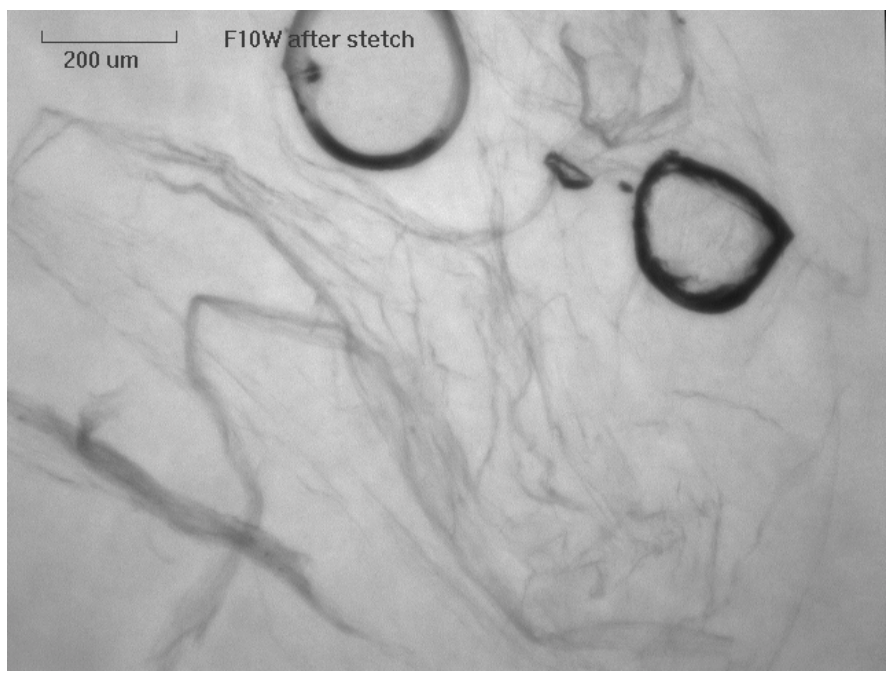

b.
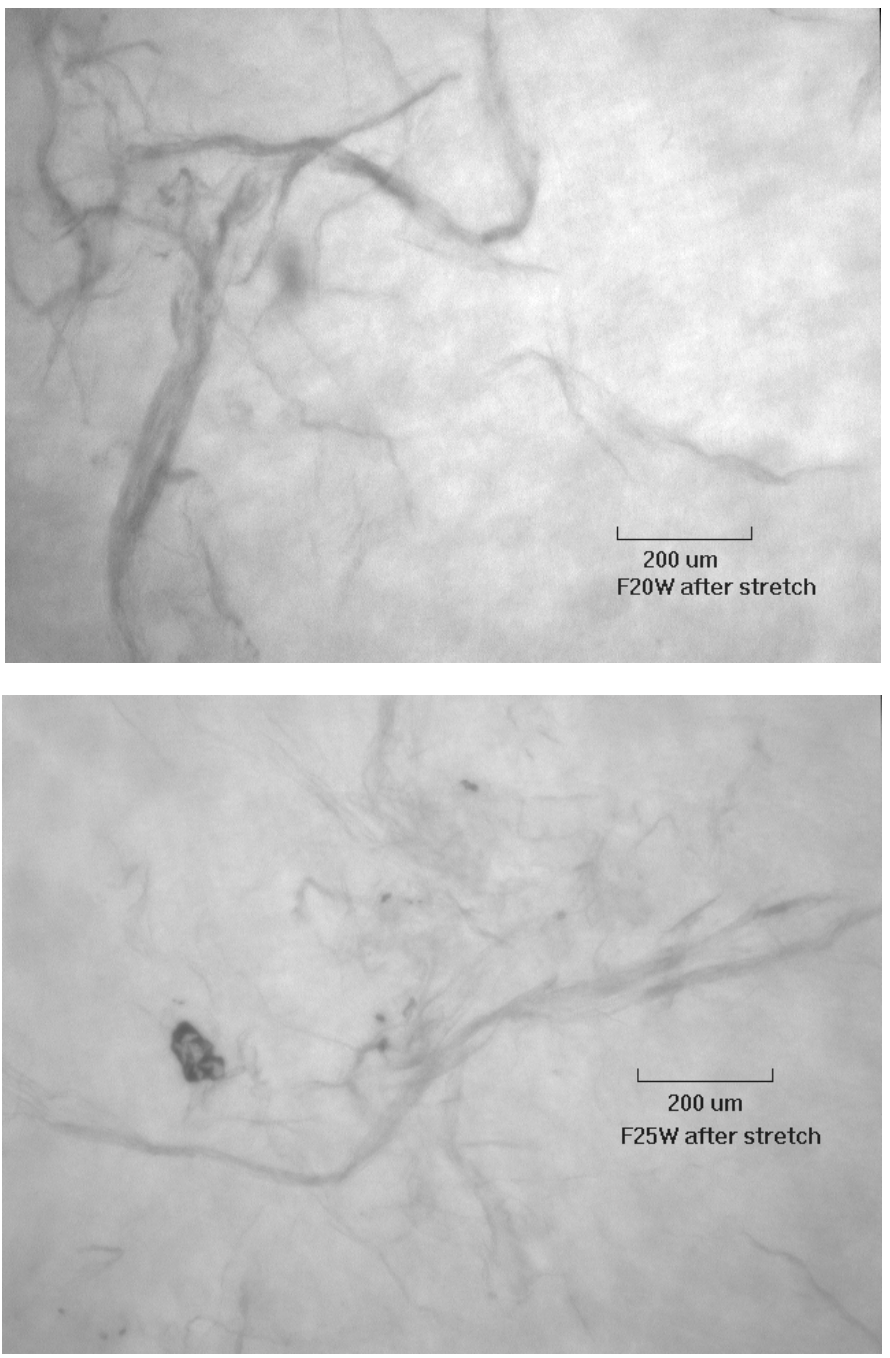

Figure 4.22 Typical images of 200 ppm fibrids dispersed in corn syrup solution (25\% water in weight) a:F10W; b:F20W; c:F25W 


\section{Chapter 5 Conclusions}

The shear viscosity was dominated by fibrid properties at low shear rates and by suspending medium properties at high shear rates which is consistent with a microstructure whose strength depends on the stress level imparted by the medium. The viscosity-shear rate behavior was best described by the Ellis model. The instantaneous strength of the microstructure was found to depend on the "space-filling" ability of the fibrids (determined by shape and concentration) as well as the stress level (shear rate and medium viscosity).

By a series of experiments, Einstein constants were obtained for the three different grades of fibrids. We can see from figures 4.17 and 4.18 that $\mathrm{K}_{E}(\mathrm{~F} 10 \mathrm{~W})>\mathrm{K}_{E}(\mathrm{~F} 20 \mathrm{~W})$ $>K_{E}(F 25 W)$. This trend is in accord with the trend of the aspect ratio for the three grades of fibrids. Einstein constants are about 200 times larger than the corresponding value for speres, which demonstrates their remarkable viscosity enhancement as well as the strong effect of dispersion.

The results of extensional viscosity measurement also prove that fibrids have tremendous enhancement effect on suspension viscosity. All the extensional viscosity data fit the following trend. F10W $>\mathrm{F} 20 \mathrm{~W}>\mathrm{F} 25 \mathrm{~W}$. This trend fits for the order of aspect ratio for the three grades of fibrids that we expected. It has been shown that extensional viscosity decreases with increasing stretch rate. This means that the fibrid suspensions "stretch thin".

According to the shear and stretch response results, fibrids have tremendous enhancement ability on the suspension viscosity, which is associated closely with their microstructure. The microstructure decides the rheological properties of fibrid suspensions. 


\section{Chapter 6 Recommendations}

Future work should be done for the verification of the extensional viscosity results. A measurement should be done for a Newtonian system to check that the Trouton ratio (ratio of the extensional viscosity to the zero-shear viscosity) does equal the theoretical value of 3.0 .

Also, the effect of fibid concentration, flow rate and filament length between the lower and upper capillary to extensional viscosity needs to be studied to get a better understanding about the extensional viscosity. 


\section{Bibliography}

Barnes, H. A., (1995) A review of the slip (wall depletion) of polymer solutions, emulsions and particle suspensions in viscometers: its cause, character, and cure, J. Non-Newtonian Fluid Mech. 56: 221-251.

Barnes, H. A., (1997) Thixotropy - a review, J. non-Newt. Fluid Mech. 70: 1-33.

Barron, J. A., (1996) New forms of matter for thixotropy and reinforcement, J. Adhes. Sealant Counc. 1: 397-423.

Barthel, H., Roseh, L. and Weis, J., (1996) Fumed silica - production, properties and applications, in: N. Auner and J. Weis, eds. Organosilicon Chemistry II, $2^{\text {nd }}$ edition. VCH Publishers, Weinheim Country of Publication, pp. 761-778.

Batchelor, G.K., (1971) The stress generated in a non-dilute suspension of elongated particles by pure straining motion, J. Fluid Mech. 46:813-829.

Becraft, M.L. and Metzner., A.B., (1992) The Rheology, fiber orientation, and processing behavior of fiber-filled fluids, J. Rheol. 36: 143-174.

Bennington, C.P.J., Kerekes, R.J. and Grace, J.R., (1990) The yield stress of fiber suspensions, Can. J. Chem. Eng. 68: 748-757.

Bird, R. B., Stewart, W. E., and Lightfoot, E. N., (1960) Transport Phenomena, Wiley, New York.

Cab-O-Sil Properties and Functions, (1987) Cabot Corporation, Tuscola, III

Carreau, P.J. , (1972) Pheological equations from molecular network theories. Trans. Soc. Rheol. 16, 99-127. 
Chan, R. C., Gupta, R. K. and Sridhar, T., (1988) Fiber spinning of very dilute solution of polyacrylamide in water, J. Non-Newt. Fluid Mech. 30, 267-283.

Chong, J.S., Christiansen, E.B., and Baer, A.D., (1971) Rheology of Concentrated Suspensions, J. Appl. Polym. Sci. 15: 2007-2021.

Cross, M. M., (1965) Rheology of non-Newtonian fluids: a new flow equation for pseudeplastic system. J. Colloid Sci. 20, 417-437.

Crowson, R.J., Folkes, M.J. and Bright, P.F., (1980A) Rheology of short glass fiber-reinforced thermoplastics and its application to injection molding I. Fiber motion and viscosity measurement, Polym. Eng. Sci. 20: 925-933.

Crowson, R.J. and Folkes, M.J., (1980B) Rheology of short glass fiber-reinforced thermoplastics and its application to injection molding II. The effect of material parameters, Polym. Eng. Sci. 20: 934-940.

Dealy, J. M., (1982) Rheometers for Molten Plastics, Van Nostrand Reinhold, New York.

Dinh, S.M. and Armstrong, R.C., (1984) A rheological equation of state for semiconcentrated fiber suspensions, J. Rheol. 28: 207-227.

Doraiswamy, D., (1998) Central R\&D work report, 177.

Einstein, A., (1956) Investigations on the Theory of the Brownian Movement, Dover, New York, pp. 36-54.

Frankel, N.A. and Acrivos, A., (1967) On the Viscosity of a Concentrated Suspensions, Chem. Eng. Sci. 22: 847-853. 
Gautier, S., Champion, E., Bernache-Assolant, D. and Chartier, T., (1999) Rheological characteristics of alumina platelete-hydroxyapatite composite suspensions. J. Eur. Ceram. Soc., 19(4), 469-477.

Goddard, J.D., (1976A) Tensile stress contribution of flow-oriented slender particles in non-Newtonian fluids, J. Non-Newtonian Fluid Mech. 1: 1-17.

Goddard, J.D., (1976B) The stress field of slender particles oriented by a nonNewtonian extensional flow, J. Fluid Mech. 78: 177-206.

Goddard, J.D., (1978) Tensile behavior of power-law fluids containing oriented slender fibers, J. Rheol. 22: 615-622.

Good, R.J., and Gupta, R.K., (1991) The Coupling of Interfacial, Rheological, and Thermal Control Mechanisms in Polymer Adhesion, Adhesive Bonding (L. H. Lee, ed.), Plenum Press, New York, pp. 47-73.

Gupta, R.K., (2000) Polymer and Composite Rheology (Second Edition, Revised and Expanded), Marcel Dekker, New York.

Gupta, R.K., Sridhar, T., (1984), Advances in Rhelogy, Vol 4: Applications, Universidad Nacional Autonomas de Mexico, pp. 71.

Gupta, R.K., and Sridhar, T., (1998) Elongational Rheometers, Rheological Measurement, Sencond ed. (A. A. Collyer and D.W. Clegg, eds.), Chapman and Hall, London, pp. 516-549.

Happel, J. and Brenner, H., (1983) Low Reynolds Number Hydrodynamics, Martinus Nijhoff, Dordecht, pp. 431-473.

Jeffrey, G. B., (1922) The Motion of Ellipsoidal Particles Immersed in a Viscous Fluid, Proc. Roy. Soc. A102:161-179. 
Kataoka, T., Kitano, T., Sasahara, M., and Nishijima, K., (1978) Viscosity of Particle Filled Polymer Melts, Rheol. Acta 17: 149-155.

Khagram, M., Gupa, R.K., Sridhar, T., (1985) J. Rheol. 29: 191

Khan, S.A., and Zoeller, N.J., (1993) Dynamic rheological behavior of flocculated fumed silica suspensions, J. Rheol., 37: 1225-1235.

Kitano, T., Kataoka, T., and Shirota, T., (1981) An Empirical Equation of the Relative Viscosity of Polymer Melts Filled with Various Inorganic Fillers, Rheol. Acta 20: 207-209.

Liang, R.F., Han, L., Doraiswamy, D. and Gupta, R.K., (2001) The Rheology of Aramid Platelet, submitted to Chemical Engineering Science.

Maxwell, B., and Chartoff, R. P., (1965) A Polymer Melt in an Orthogonal Rheometer, Trans. Soc. Rheol. 9: 41-52.

Mewis, J., and Metzner, A.B., (1974) The rheological properties of suspensions of fiber in Newtonian fluids subjected to extensional deformations, J. Fluid Mech. 62:593600.

Petrie, C. J. S., (1919) Elongational Flows, Pitman, London.

Pittman, J.F.T., and Bayram, J., (1990) Extensional flow of polydisperse fiber suspensions in free-falling liquid jets, Int. J. Multiphase Flow 16: 545-559.

Poslinski, A.J., Ryan, M.E., Gupta, R.K., Seshadri, S.G., and Frechette, F.J., (1988) Rheological Behavior of Filled Polymer Systems I. Yield Stress and Shear-Thinning Effects, J. Rheol. 32: 703-735. 
Poslinski, A.J., Ryan, M.E., Gupta, R.K., Seshadri, S.G., and Frechette, F.J., (1988) Rheological Behavior of Filled Polymer Systems II. The Effect of a Bimodal Size Distribution of Particulates, J. Rheol. 32: 751-771.

Rees, S., (1995) New developments in thixotropic coatings, Polym. Paint Colour J. 185 (4369): 10-11.

Rides, M., Allen, C.R.G., and Charkravorty, S., (1996) Review of Extensional Viscoelasticity Measurement Techniques for Polymer Melts, Report \# CMMT(A)44, National Physical Laboratory, Teddington, U. K.

Technical Bulletin Pigments No. 23, (1989) Degussa Corporation, Allendale, NJ

Sridhar, T. and Gupta, R.K., (1985) A simple extensional viscometer, Rheol. Acta 24, 207-209.

Sridhar, T. and Gupta, R.K., (1986) Application of the Batchelor theory to fiber spinning of suspensions, Proc. $4^{\text {th }}$ National Conf. on Rheol., Adelaide, Australia, pp. 185-190.

Sundararajakumar, R. R., Koch, D. L. and Shaqfeh, E. S. G., (1994) Extensional viscosity and effective thermal conductivity of a dispersion of aligned disks, Phys. Fluids, 6, 1955-1962.

Tucker, C.L. and Advani, S.G., (1994) Processing of short-fiber systems, Flow and Rheology in Polymer Composite Manufacturing (Advani, S.G., ed.), Elsevier, Amsterdam, pp. 147-202.

Vaxman, A., Narkis, M., Siegmann, A., and Keing, S., (1989) Short-fiber-reinforced thermoplastics. Part III: Effect of fiber length on rheological properties and fiber orientation, Polym. Compos. 10: 454-462.

Walters, K., (1975) Rheometry, Chapman and Hall, London. 
Williams, D. J., (1971) Polymer science and engineering, Prentice-Hall, Canada.

Wu, S., (1979) Order-disorder transitions in the extrusion of fiber-filled poly (ethylene terephthalate) and blends, Polym. Eng. Sci. 19: 638-650.

Yziquel, F., Carreau, P. J., Moan, M. and Tanguy, P. A., (1999) Rheological modeling of concentrated colloidal suspensions, J. non-Newtonian Fluid Mech., 86, 133-155. 


\section{Appendix A Conference Publication}

R. Liang, L. Han, D. Doraiswamy and R. K. Gupta, "Fundamental Characterization of Structured Fibrid Suspensions", Proc. XIllth Intl. Congress on Rheology, Cambridge, UK, $\underline{4}, 136-138$ (2000) 


\title{
FUNDAMENTAL CHARACTERIZATION OF "STRUCTURED" FIBRID SUSPENSIONS
}

\author{
RUifeng Liang ${ }^{1}$, Long HaN ${ }^{1}$, DeEpaK Doraiswamy ${ }^{2}$ and RAKeSh K. GuPta ${ }^{1}$ \\ ${ }^{1}$ Department of Chemical Engineering, West Virginia University, P.O. Box 6102, Morgantown, WV 26506, USA \\ ${ }^{2}$ DuPont Central R\&D, Experimental Station, P.O. Box 80302, Wilmington, DE19880-0302, USA.
}

\begin{abstract}
Aramid fibrids are believed to form microstructures in suspensions at very low loading levels $(\sim 0.5 \%)$ because of the "space-filling" nature of their shapes and thus provide a convenient means of introducing yield stress behavior for various commercial applications. The effect of fibrid shape, concentration and medium (corn syrup/water) viscosity on the dynamic, steady and transient shear responses was determined using a parallel plate geometry.
\end{abstract}

KEYWORDS: NOMEX® FIBRIDS, STRUCTURED SUSPENSIONS, DYNAMIC YIELD STRESS, THIXOTROPY

\section{INTRODUCTION}

Nomex® aramid particulates like fibrids induce yield stresses in various suspensions by the addition of very small amounts $(\sim 0.5 \%)$ for potential applications such as sealants, adhesives, roofing and roof coatings, thick film coatings, aqueous latex paints and caulks; fumed silica behaves similarly. The desired properties for these applications include a high viscosity (or yield stress behavior) at low shear rates for sag or slump resistance and a low viscosity at high shear rates for good processability in operations like mixing, pumping, painting and spraying. These characteristics are a result of the rectangular platelet shape of these fibrids $(\sim 100 \times 100 x 0.1 \mu \mathrm{m})$ which is rarely approached in most situations because of their tendency to form coils (see Figure 1). In addition, these additives also impart other properties like reinforcement, chipping resistance and surface finish because of the chemical nature of the Nomex® polymer (poly(isophthaloyl-chloride/metaphenylene-diamine)).

Fibrids with three different morphologies were used to prepare "structured" suspensions and dynamic, steady shear and transient responses of these suspensions were investigated comprehensively. The roles of fibrid properties, fibrid concentration and the dispersion medium viscosity were evaluated. The results were correlated with suspension microstructure and dispersability which are critical aspects in their commercialization.

\section{EXPERIMENTAL DETAILS}

Three Nomex® fibrids with different morphology were used in this work which are referred to as F10, F20 and F25 as per DuPont terminology; the increasing number reflects increasing levels of mechanical work done on the fibrids in a refining process. The fibrids were provided in wet form with varying water content which were then suspended in a commercial grade corn syrup; the corn syrup was diluted with different levels of water to obtain varying levels of
Newtonian behavior $(7.4,2.45$ and $0.70 \mathrm{P}$ for water concentrations of $7.1,13.8$ and $23.9 \%$ respectively at 25 C). Three weight concentrations $(0.18 \%, 0.54 \%$ and $0.80 \%)$ were considered for each fibrid shape and viscosity level so that a total of 27 formulations were studied.
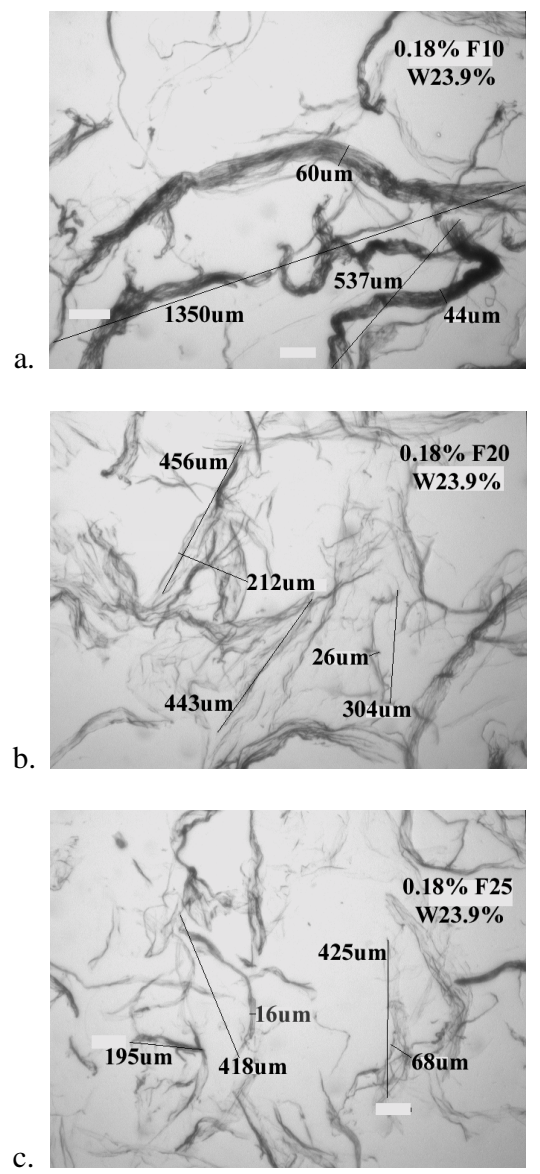

Figure 1. Typical images of $0.18 \%$ fibrids dispersed in corn syrup with $23.9 \%$ water ; a: F10, b: F20, c: F25.

To facilitate dispersion, the fibrids were separated by hand and then dispersed at high concentration (1.2\%) in pure corn syrup (with a viscosity of $39.1 \mathrm{P}$ ) for 3 minutes using an Arrow 1750 motorized stirrer to form a master solution. The dispersion quality was verified by visual inspection under a microscope. A masterbatch of $1400 \mathrm{gm}$ was prepared for each type of fibrid from which the various test solutions were prepared in $150 \mathrm{gm}$ quantities by thinning down with water by high shear stirring for 2 minutes. The prepared suspensions were allowed to sit for one week to eliminate air bubbles. Typical pictures of the three fibrids at one concentration are shown in Figure 1. The F20 fibrids are seen to be more uniformly distributed and "space-filling" than the other types. 
Dynamic, transient and steady shear data were obtained on a Rheometrics RMS 800 instrument using parallel plate fixtures at $25 \mathrm{C}$. Normal stresses were not considered in view of their arbitrary behavior and a failure to reflect any clear trends. Slip effects were effectively eliminated by moving to large gaps (a $1.5 \mathrm{~mm}$ gap was used). Evaporation effects were minimized by using a thin coating of low viscosity vegetable oil. Loading and initial structure effects were eliminated by subjecting the sample to multiple strain sweeps $(2-200 \%)$ at a fixed frequency of 1 $\mathrm{rad} / \mathrm{s}$. In steady shearing experiments, pre-shearing at 0.5 $1 / \mathrm{s}$ for 1 minute was found to be essential to provide reproducible results.

Essentially constant stress values were obtained after two strain sweeps; a three-run consecutive strain sweep was therefore adopted for all samples to eliminate residual structure effects and to get reproducible results. The dynamic moduli did not attain a constant value even at strains as low as $2 \%$ indicating non-linear viscoelasticity (or possibly a linear viscoelastic region only at vanishing low strains); the loss modulus had a weaker strain dependence than the storage modulus for all the suspensions studied. To minimize this non-linear effect, a fixed strain of $5 \%$ was used in the frequency sweep for all the suspensions studied (this also satisfied torque limitations for the low viscosity samples). Only limited ultra-low frequency data at $0.001 \mathrm{rad} / \mathrm{s}$ were obtained for comparison purposes (in view of the inordinately long times ( 18 hours) required ).

Only data (figures) for suspensions of all three fibrid types at $0.54 \%$ loading in a suspending medium of viscosity $2.45 \mathrm{P}$ are reported in interests of clarity and space. Data for the other concentrations and viscosities are mentioned only where appropriate. Intrinsic viscosity and extensional viscosity results will be reported later.

\section{RESULTS AND DISCUSSIONS Dynamic storage and loss moduli}

Figure 2 indicates that the loss modulus is much lower than the storage modulus until very high frequencies are achieved after which it has the higher value; this indicates the presence of a microstructure with an associated yield stress behavior. The F20 suspension has both the largest storage modulus and loss modulus, followed by the F25 suspension and then the F10 suspension; this indicates that the microstructure formed in the F20 suspension has the highest strength. The F20 data also indicate a frequencyindependence at low frequencies, implying that the sample is far away from the yielding point and behaves as an elastic solid.

The dynamic moduli were observed to increase significantly with fibrids concentration. For the suspension containing $0.80 \%$ fibrids, the storage modulus remained unchanged over the entire range of frequency. Increasing the matrix viscosity was found to result in little change in the dynamic moduli at low frequencies but resulted in a larger loss modulus at high frequencies.

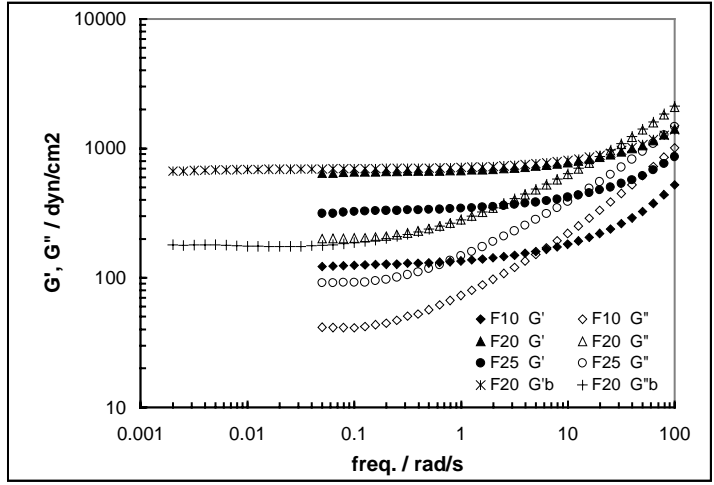

Figure 2. Dynamic moduli vs. frequency ( $\operatorname{strain}=5 \%$ ).

\section{Steady shear viscosity}

A three decade change in shear viscosity between the low shear rate region and the high shear rate region is seen for all three suspension types in Figure 3. This demonstrates the enormous influence of the addition of a tiny amount of fibrid particulates. The F20 suspension has the highest shear viscosity followed by the F 25 and the F10 suspensions and is consistent with the dynamic data. However, these suspensions do not exhibit a yield-stresstype behavior other than high zero-shear viscosities at low shear rates, which deviates from the dynamic data. For comparison, complex viscosity data are also plotted as a function of frequency in Figure 3 for these suspensions, and it is seen that the Cox-Merz rule is not valid. The slope of complex viscosity vs. frequency at low frequencies equals -1 , indicating a constant stress which may be called the "dynamic yield stress".

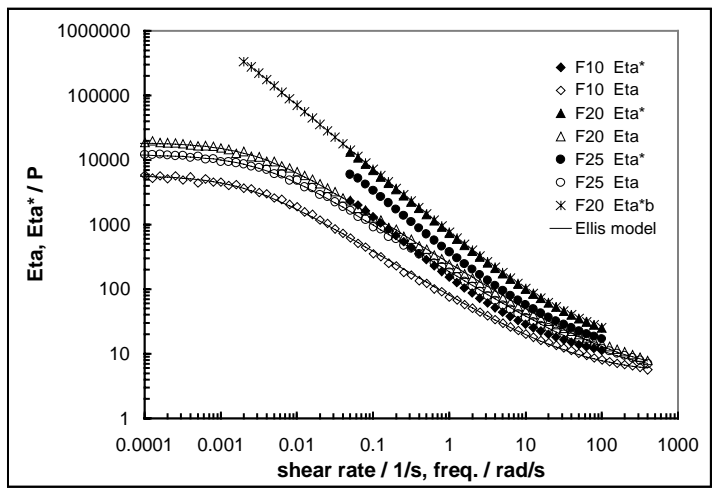

Figure 3. Shear viscosity and complex viscosity vs. shear rate or frequency; solid lines are Ellis model fits.

Figure 4 indicates that the zero-shear viscosities of the suspensions of all fibrid types are effectively independent of the suspending medium viscosity and determined only by the morphology and concentration of the fibrids. The matrix viscosity only affects the flow behavior in the shear thinning region and at very high shear rates. This behavior is also reflected in the complex viscosity data shown in Figure 4. These results indicate that the microstructure at low shear rates is governed only by the unique morphology and adjustable concentration of the fibrids; at high shear rates the microstructure might be expected to be disrupted because of the higher stresses imparted. 


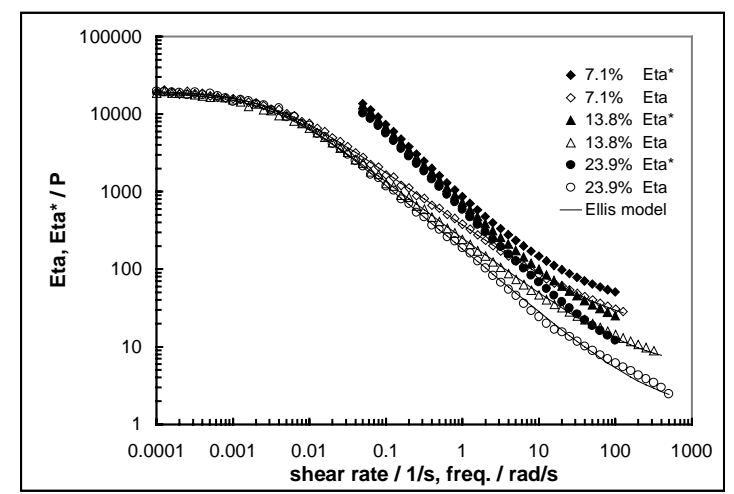

Figure 4. Effect of dispersion medium viscosity on shear viscosity and complex viscosity vs. shear rate or frequency for $0.54 \%$ F20 suspensions; solid lines are Ellis model fits.

Figures 3 and 4 clearly indicate a high zero-shear viscosity region at low shear rates, followed by a shear thinning region, and eventually a low infinite-shear viscosity region at high shear rates. The Ellis model was used to describe the viscosity behavior and the Ellis model fits are shown by the solid lines in the Figures 3 and 4 .

\section{Stress growth and decay}

Step rate experiments were used to identify microstructure evolution in the fibrid suspensions. A step shear rate of 0.5 or $1.01 / \mathrm{s}$ was applied for the first $60 \mathrm{~s}$ to observe stress growth and then stepped down to zero shear rate to observe stress decay for another $60 \mathrm{~s}$.

Typical stress growth and decay curves are shown for three suspending liquid viscosity levels in Figure 5. For step shear rate increases to the lower final shear rate (0 -> $0.51 / \mathrm{s}$ ) the suspensions show stress growth curves that reach equilibrium with the equilibrium stress increasing with the matrix viscosity. In the subsequent stress decay experiment $(0.5 \rightarrow 0)$ there is an instant small initial drop after which the stress stays at a constant high level which increases with increasing matrix viscosity. This response is quite unlike that of a Newtonian or typical viscoelastic fluid. It was observed that the residual stress level depends on the morphology of the fibrids and their concentration.

In the higher shear rate experiments at $1.01 / \mathrm{s}$, during the step increase phase from 0 to $1.01 / \mathrm{s}$, the suspensions first show a weak stress overshoot before attaining an equilibrium stress value. However, in the subsequent step decrease phase from 1.0 to $01 / \mathrm{s}$, the material response again strongly depends on the composition.

These results indicate the presence of a microstructure which is destroyed by shearing. At low stress levels (corresponding to low shear rate and/or low medium viscosity) the microstructure is weakened but not completely destroyed which results in a lower level residual stress. At high stress levels, the microstructure is destroyed and there is no residual stress. Finally, at intermediate stress levels the microstructure undergoes gradual destruction resulting in a stress decay.

The strength of the microstructure as reflected in the residual stresses depended on the fibrid type and concentration. At the same concentration, F20 samples gave stronger structures than F25 and F10 samples as also reflected in the steady shear and dynamic data.

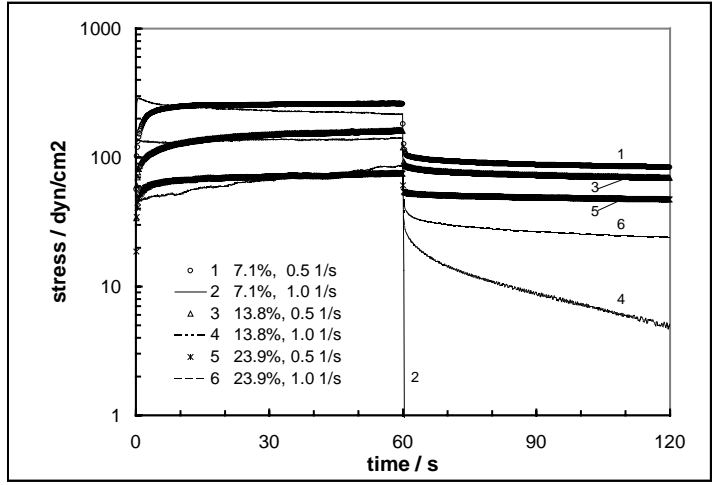

Figure 5. Stress growth and decay curves from step shear rate tests at 0.5 and $1.0 \mathrm{~s}^{-1}$ in three dispersion media for $0.54 \%$ F20 suspensions.

\section{Thixotropic loops}

Thixotropy describes a time dependent material response (typically viscosity) associated with reversible changes in the microstructure of fluids. Thixotropy was measured by ramping up the shear rate from zero to $20 \mathrm{1} / \mathrm{s}$ and then ramping down from $201 / \mathrm{s}$ to zero for another 60 seconds and determining the area inside the envelope.

The F10 suspensions were found to exhibit more thixotropy (as compared to the F20 sample) in spite of the lowest values of the dynamic moduli and viscosity; no obvious thixotropy was observed for the F25 samples. This result can be ascribed to the different morphology and dispersability of the fibrids. Poorly dispersed F10 samples take relatively longer time to build up the microstructure destroyed by shearing. It is also concluded from experimental data that thixotropy increases with fibrids concentration; increasing the matrix viscosity has little effect on thixotropy and at very high levels can depress it because of the larger disruptive stresses imparted to the microstructure.

\section{Conclusions}

Dynamic yield behaviors at low frequency but high zero-shear viscosity plateaus at low shear rates were observed. The shear viscosity was dominated by fibrid properties at low shear rates and by suspending medium properties at high shear rates which is consistent with a microstructure whose strength depends on the stress level imparted by the medium. The viscosity-shear rate behavior was best described by the Ellis model. The fibrid with the most space-filling geometry (F20) had the highest values of the dynamic moduli and zero-shear viscosity. The instantaneous strength of the microstructure was found to depend on the "space-filling" ability of the fibrids (as determined by shape and concentration) as well as the stress level (shear rate and medium viscosity).

\section{ACKNOWLEDGEMENT}

This work was funded, in part, by the DuPont company. 
Appendix B Shear Viscosity Data

Figure B1-B18

Table B1 


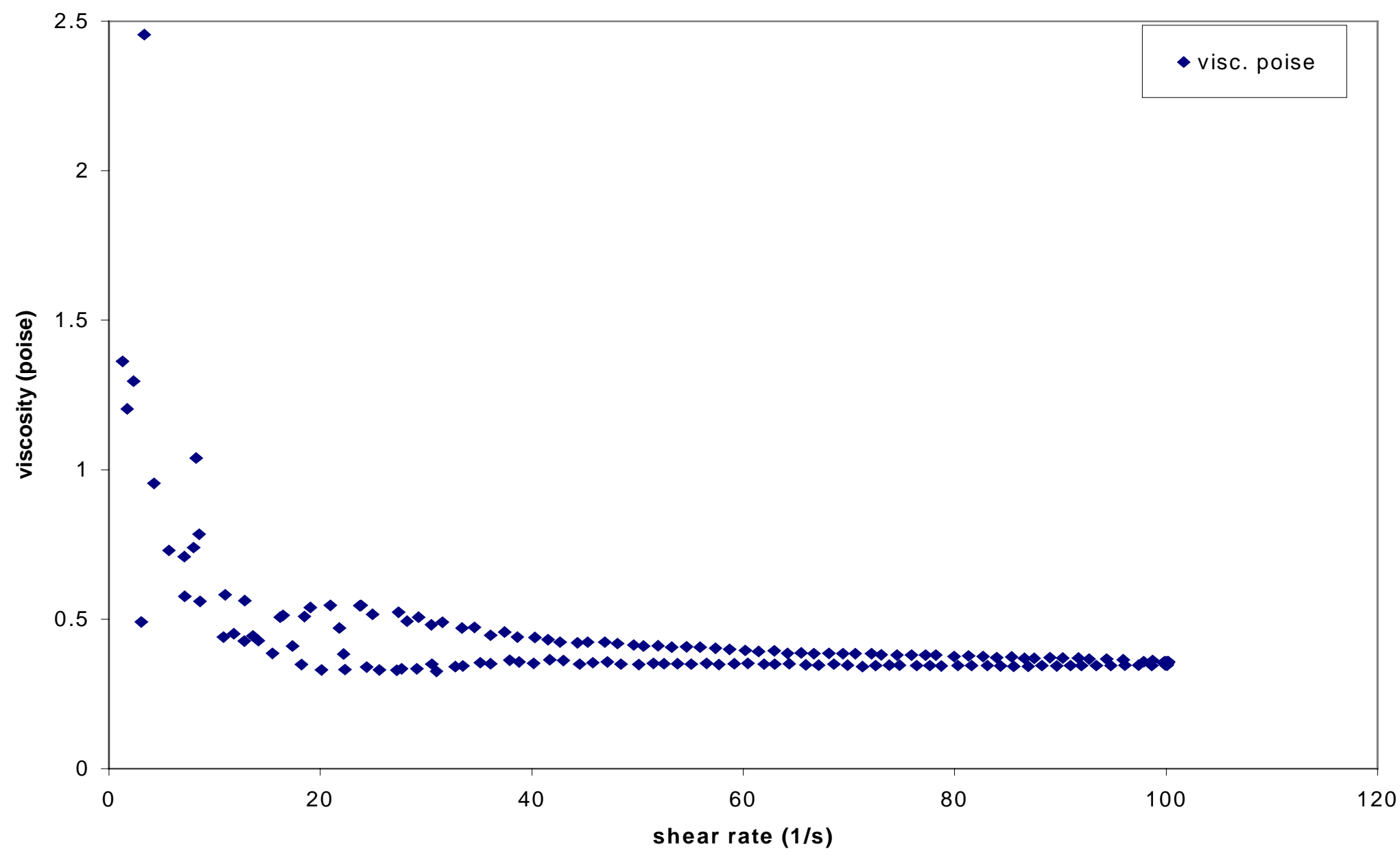

Figure B1. Viscosity vs. shear rate of

$30 \%$ water of corn syrup solution (500 micron gap) 


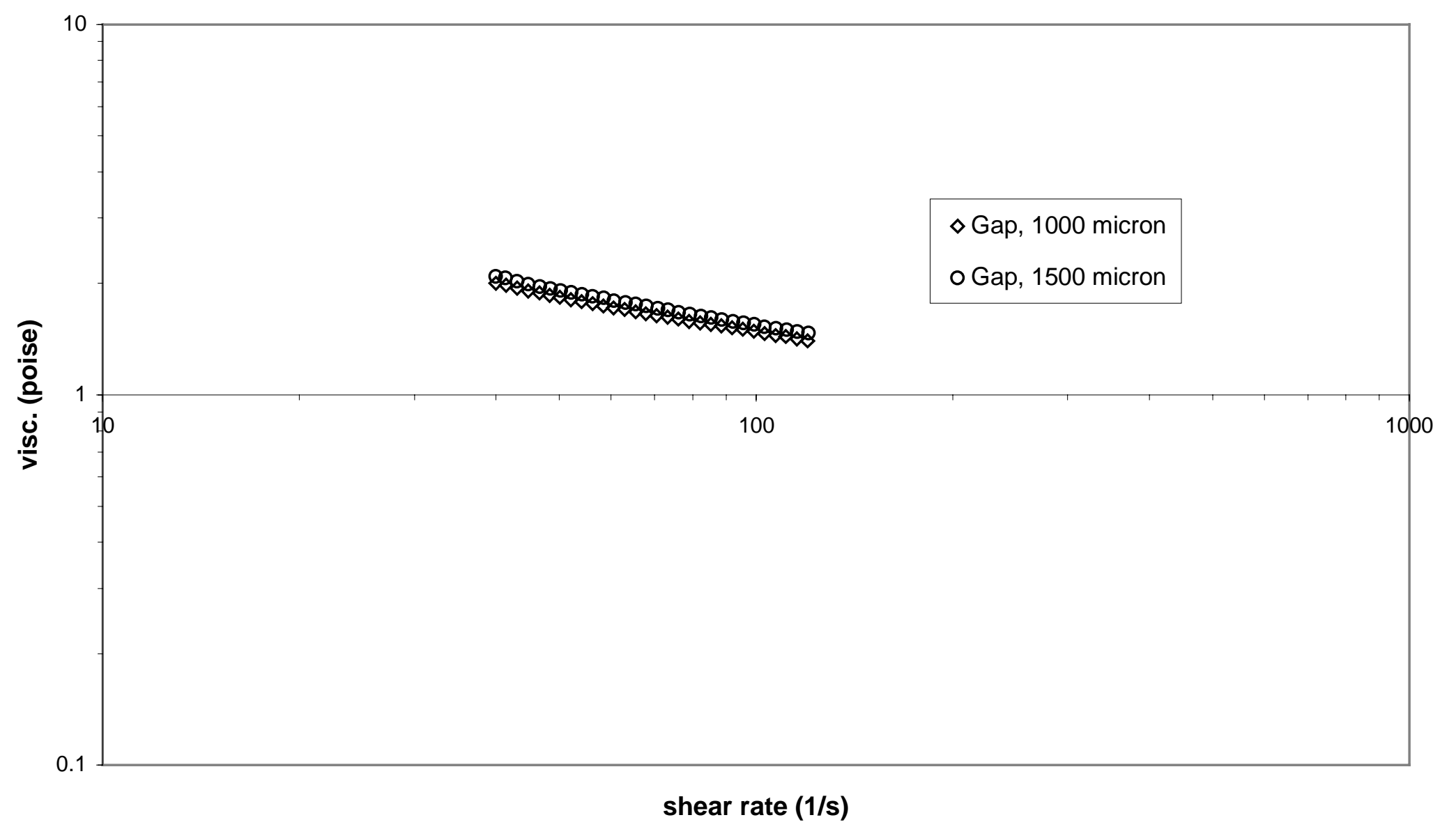

Figure B2 Gap effect for 0.18\% F20W in corn syrup solution (23.9\% water in weight) 


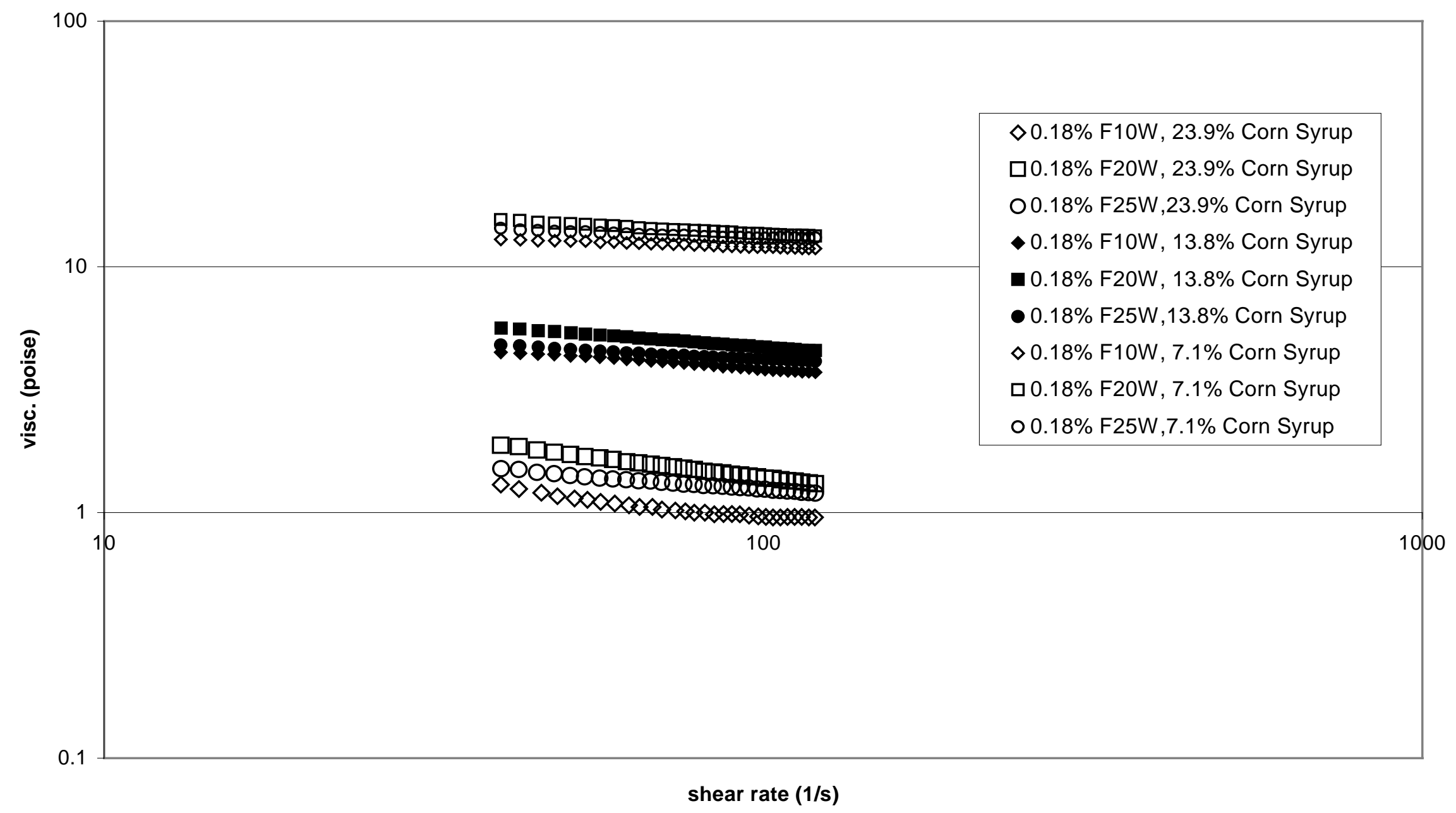

Figure B3 Visc. vs. Shear rate for $0.18 \%$ fibrid 


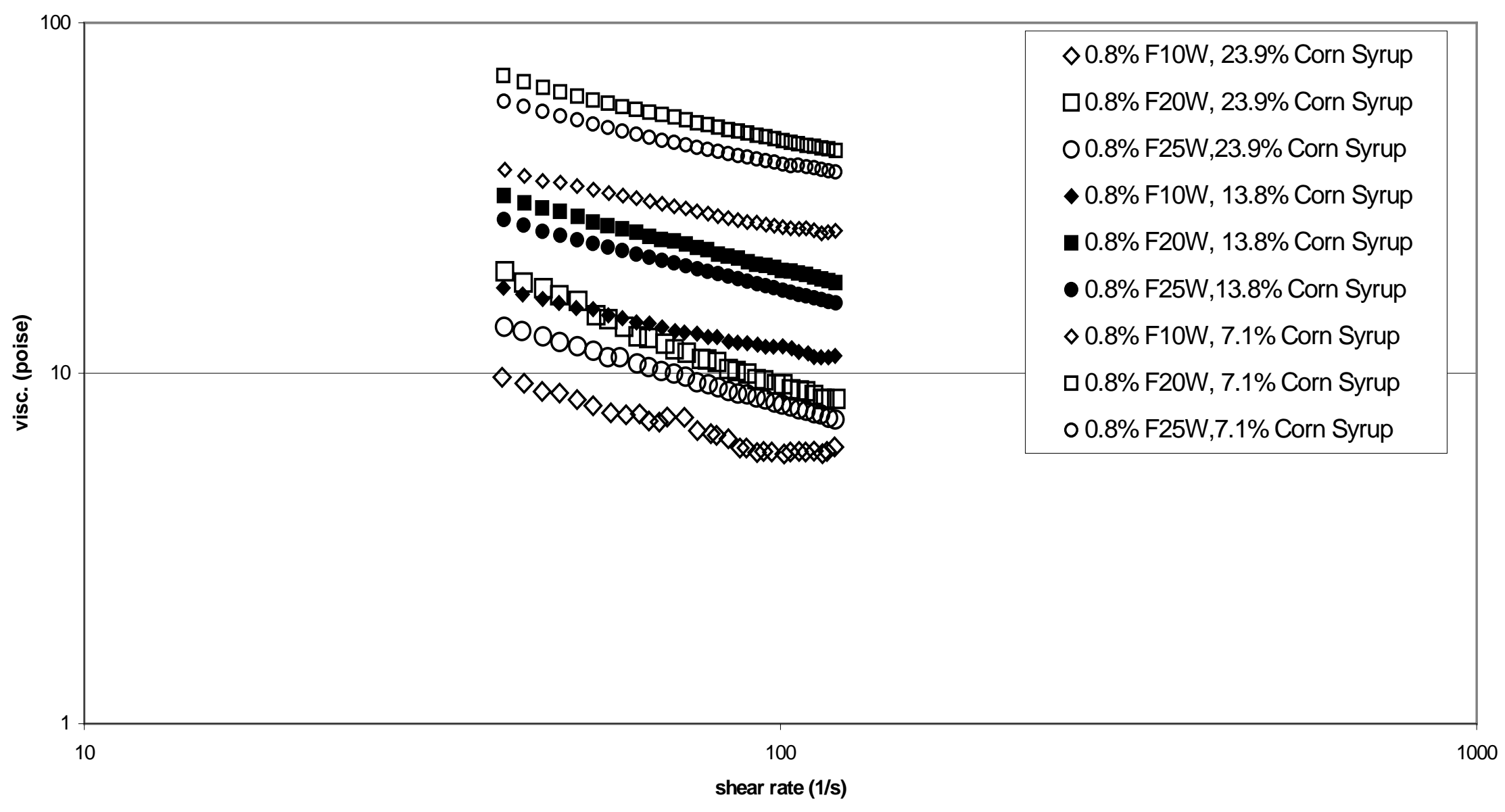

Figure B4 Visc. vs. shear rate for $0.8 \%$ fibrid 


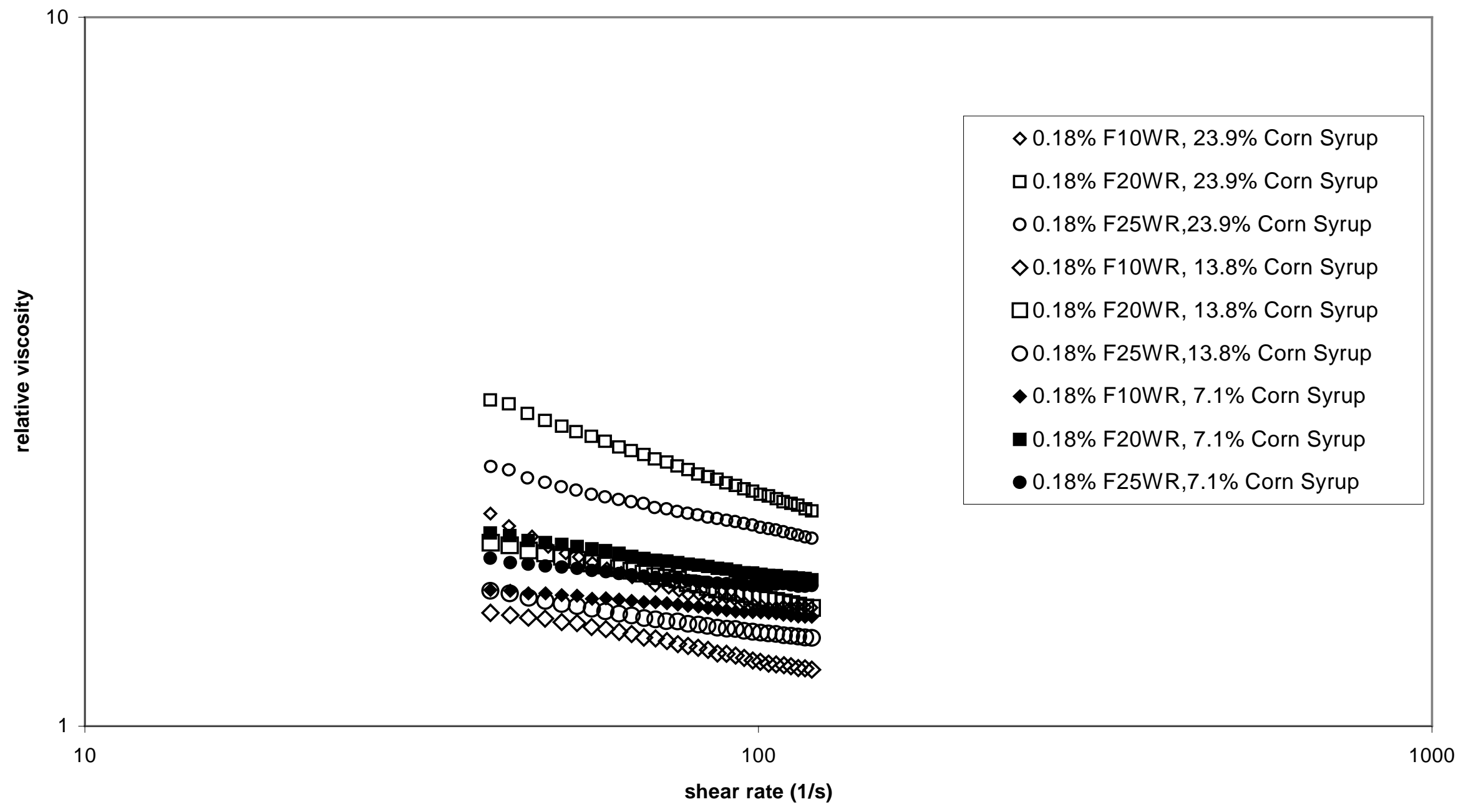

Figure B5 Relative Visc. vs. shear rate for $0.18 \%$ fibrid 


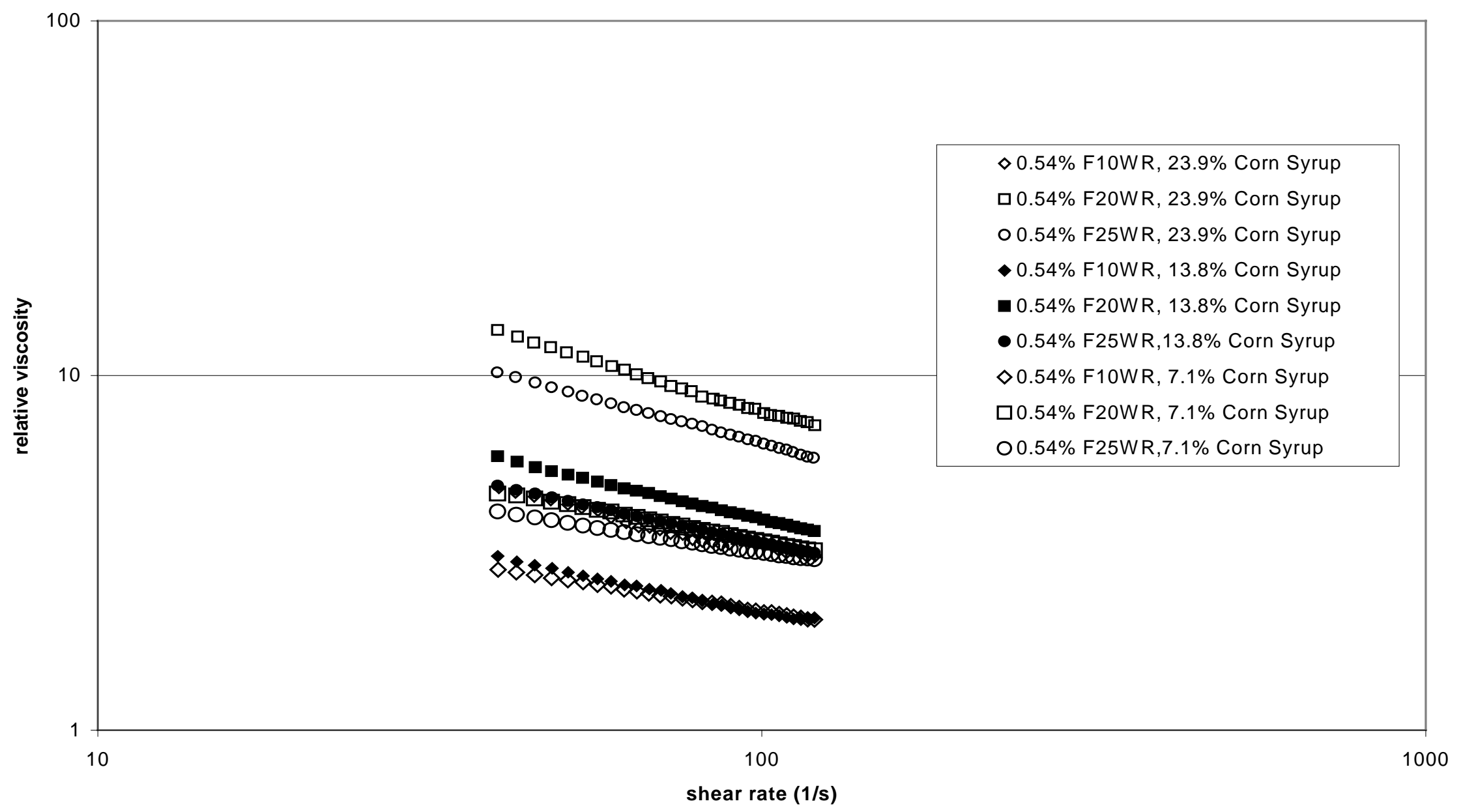

Figure B6 Relative visc. vs. shear rate for $0.54 \%$ fibrid 


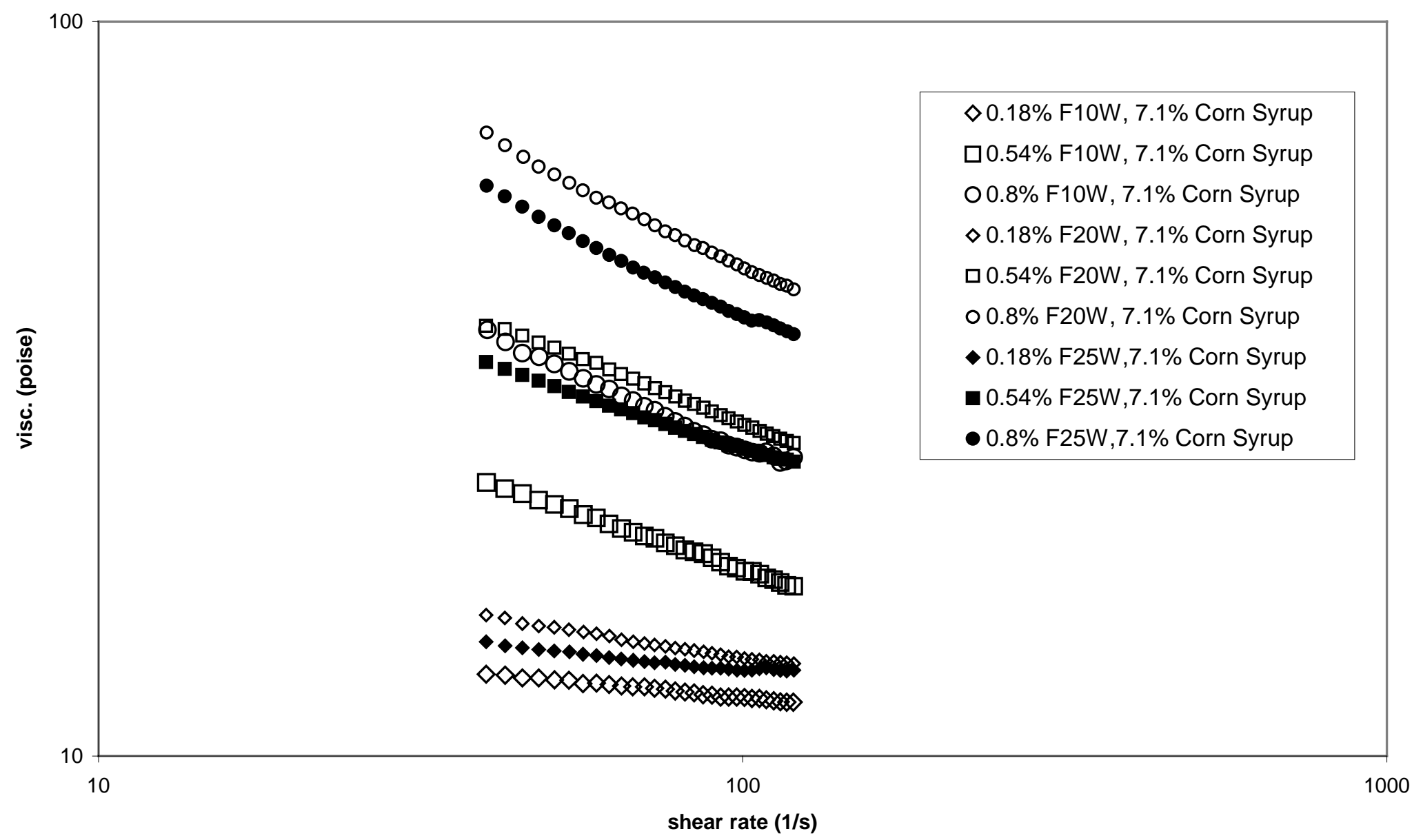

Figure B7 Visc. vs. Shear rate for $7.1 \%$ matrix fluid 


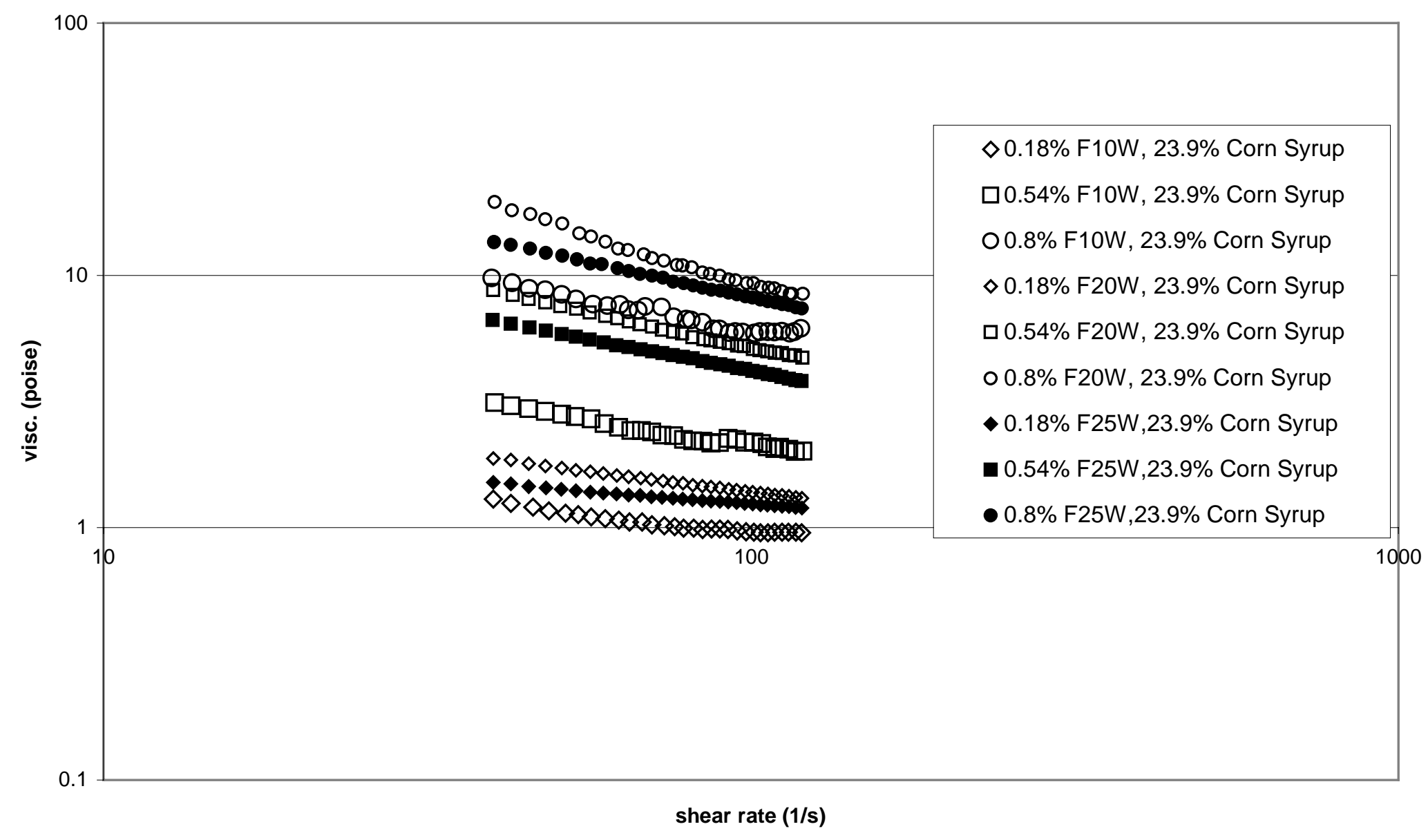

Figure B8 Visc. vs. shear rate for $23.9 \%$ matrix fluid 


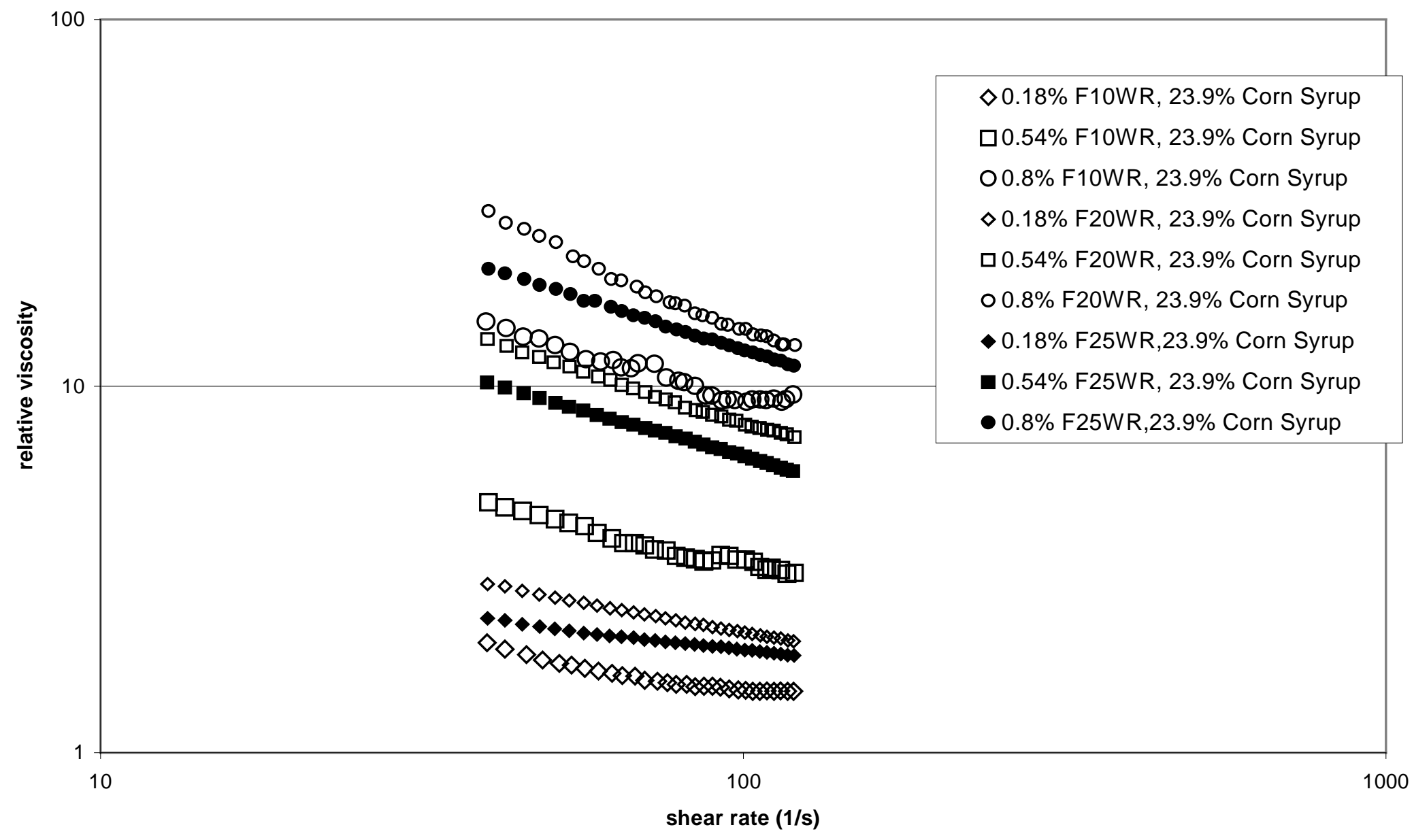

Figure B9 Relative visc. vs. shear rate for $23.9 \%$ matrix fluid 


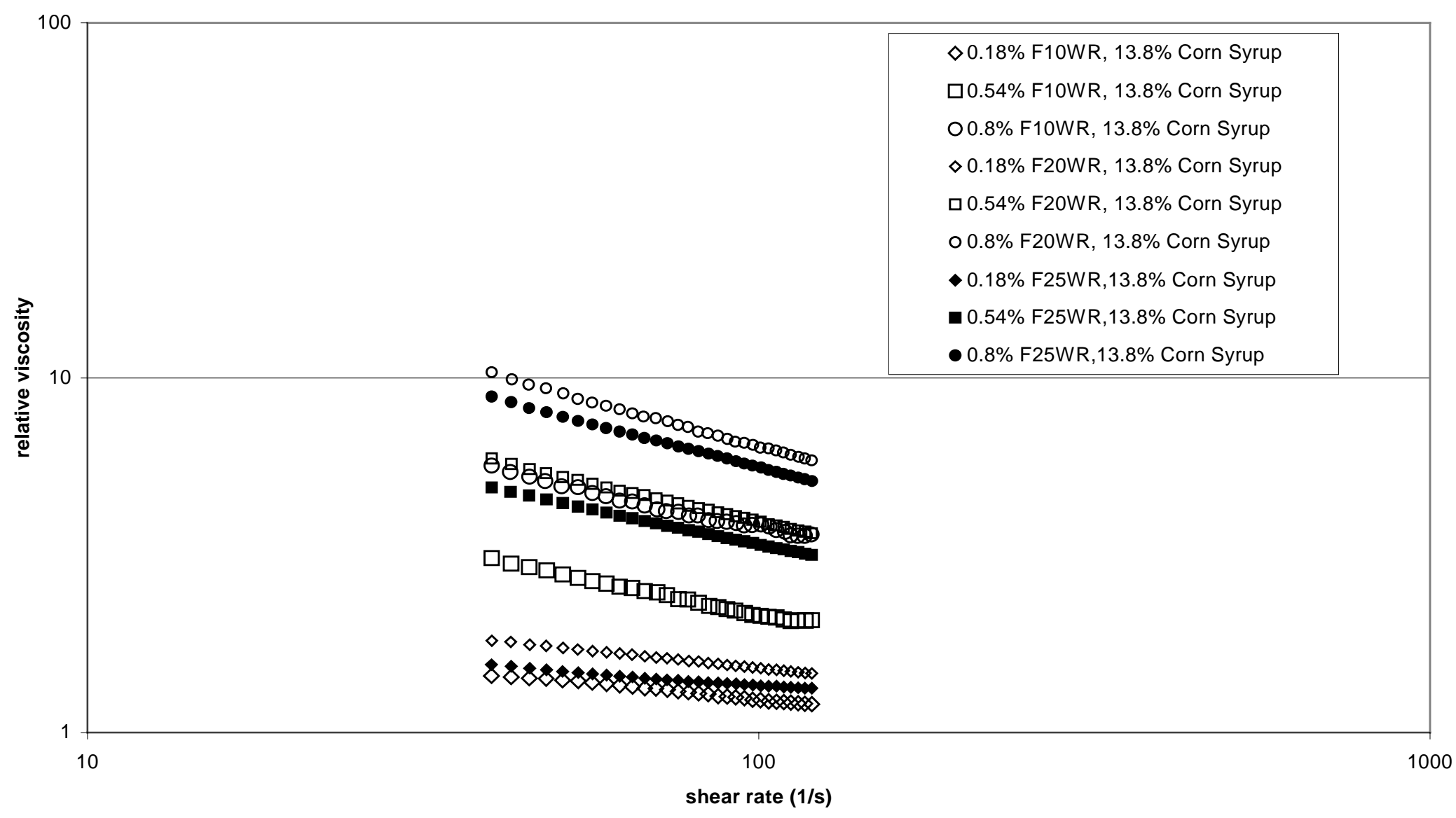

Figure B10 Relative visc. vs. shear rate for $13.8 \%$ matrix fluid 


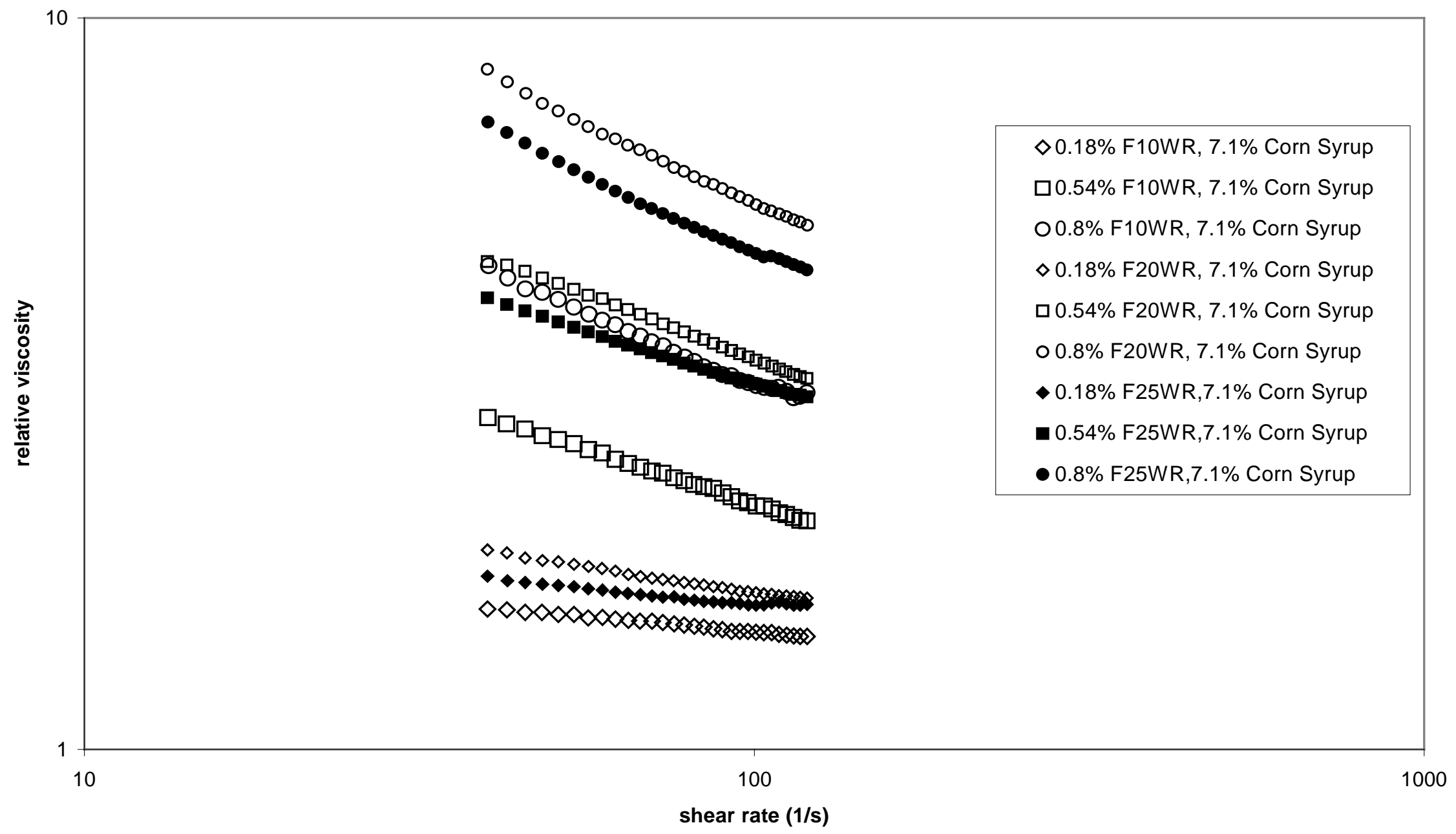

Figure B11 Relative visc. vs. shear rate for $7.1 \%$ matrix fluid 


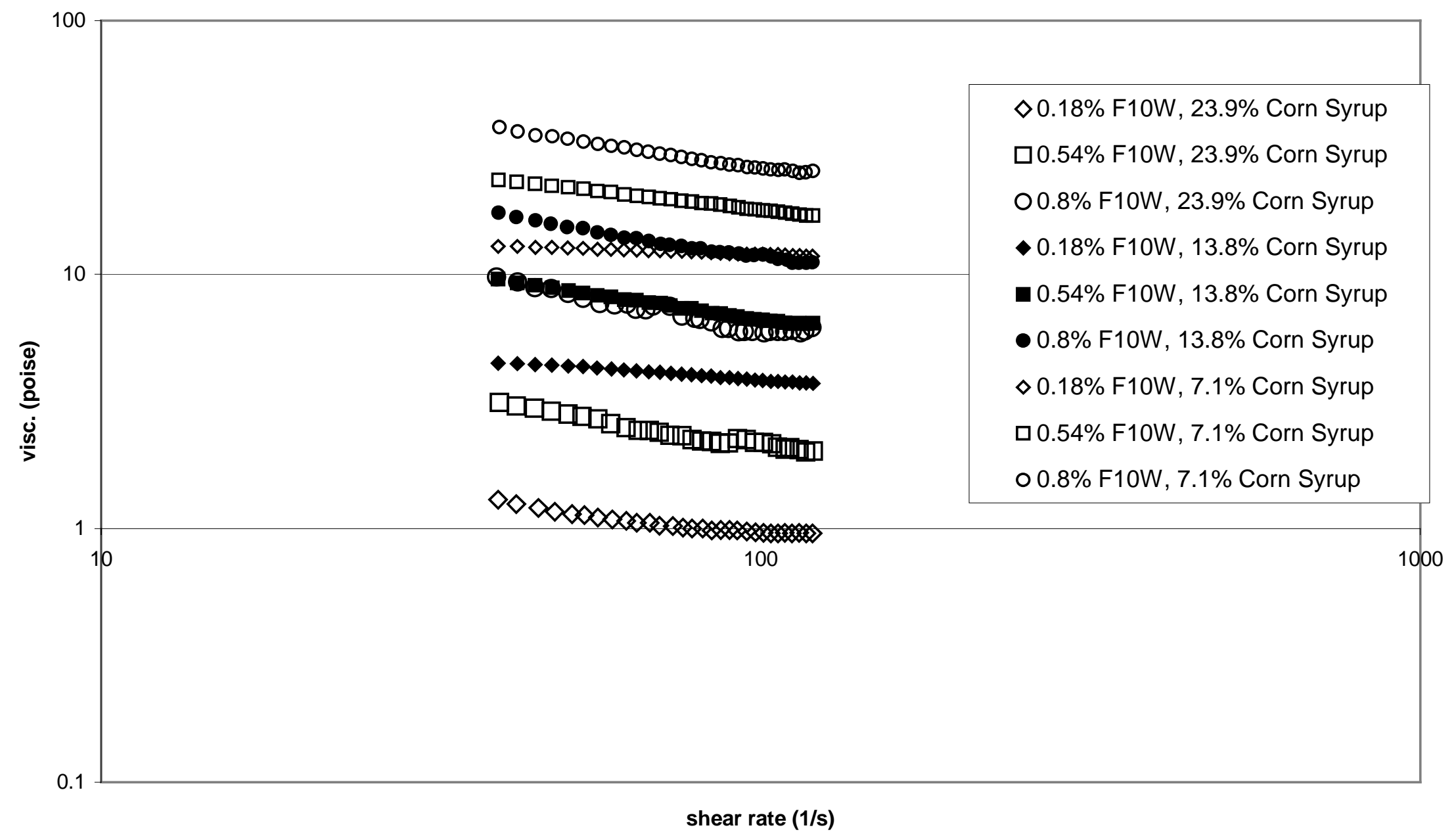

Figure B12 Visc. vs. Shear rate for F10W 


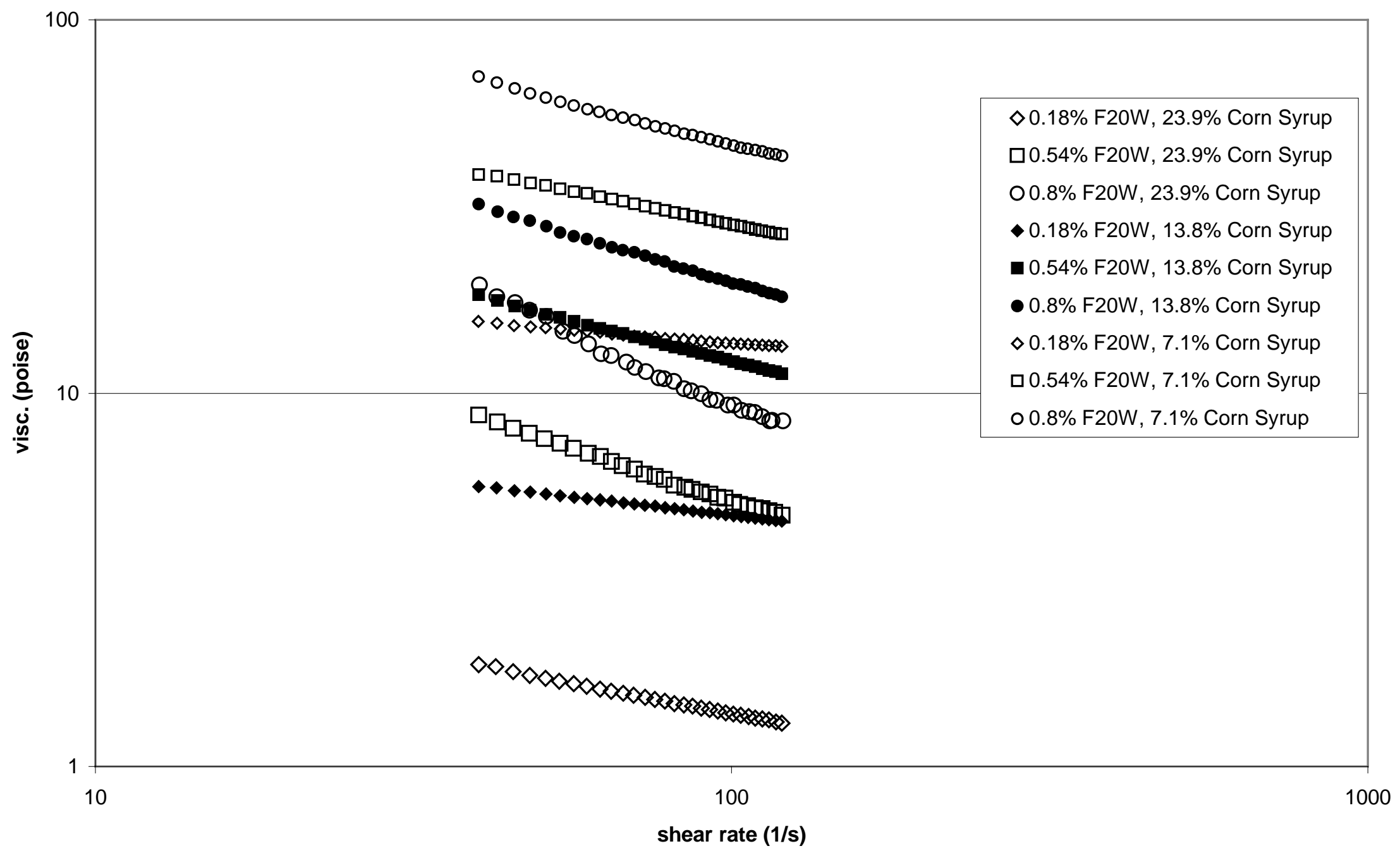

Figure B13 Visc. vs. shear rate for F20W 


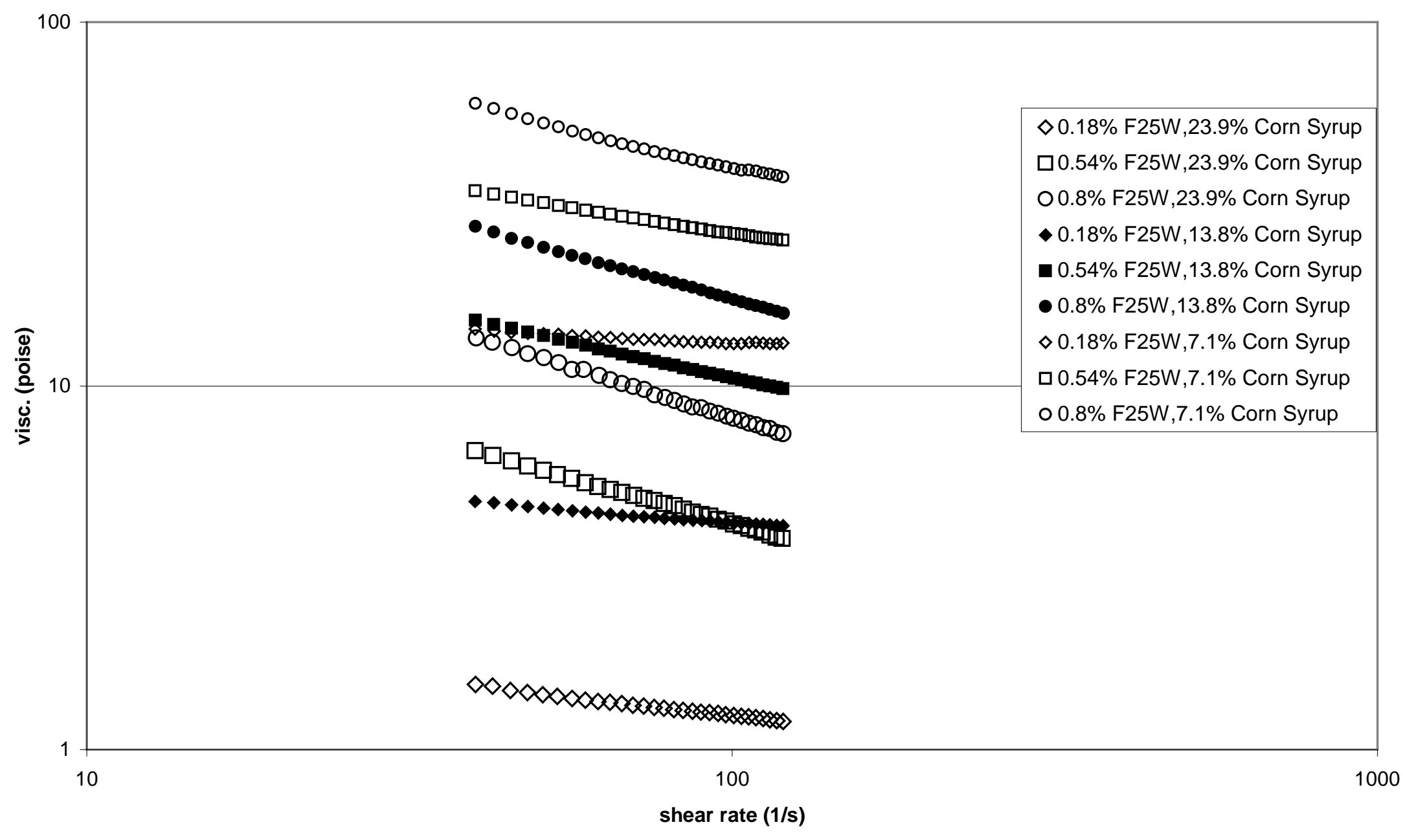

Figure B14 Visc. vs. shear rate for F25W 


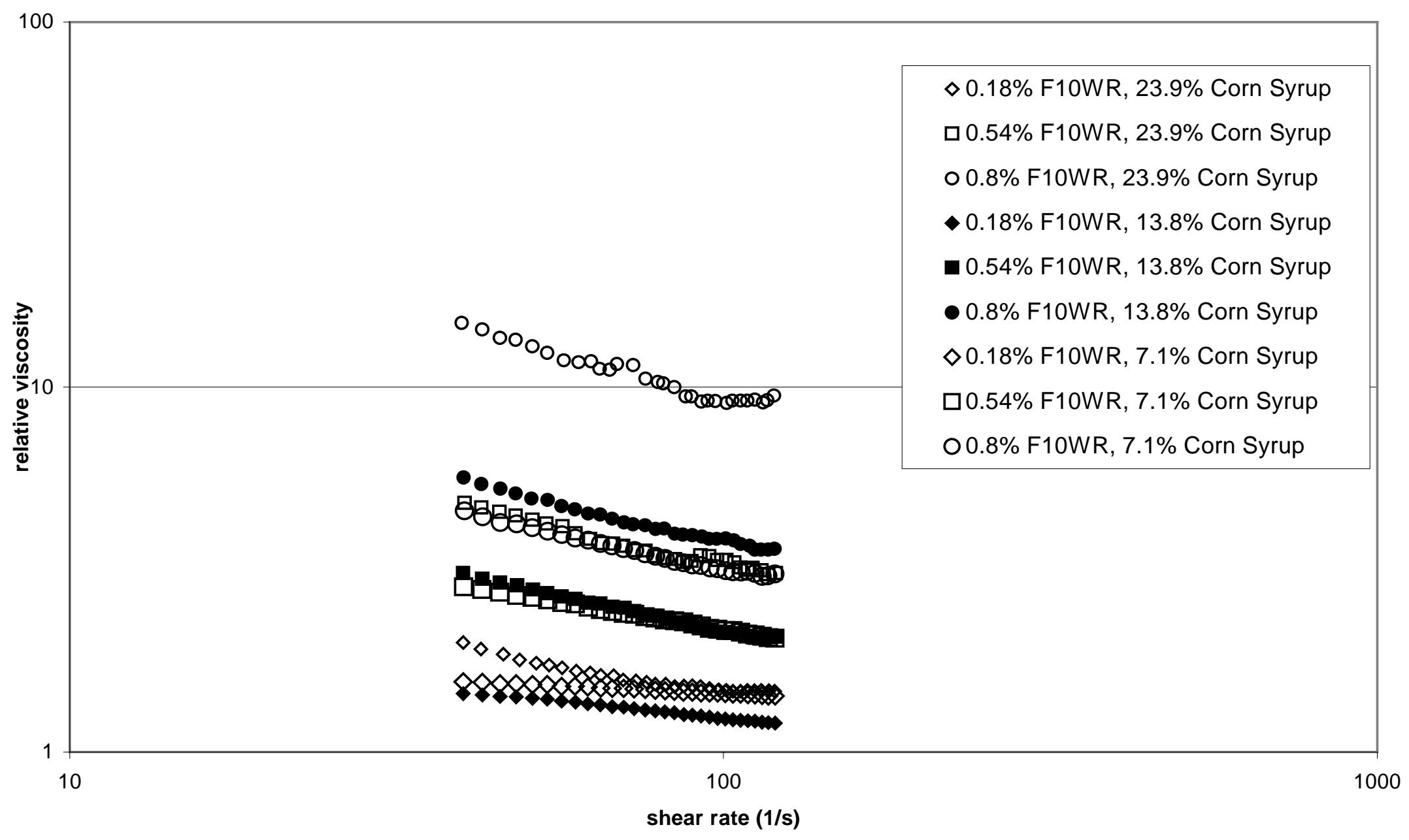

Figure B15 Relative visc. vs. shear rate for F10W 


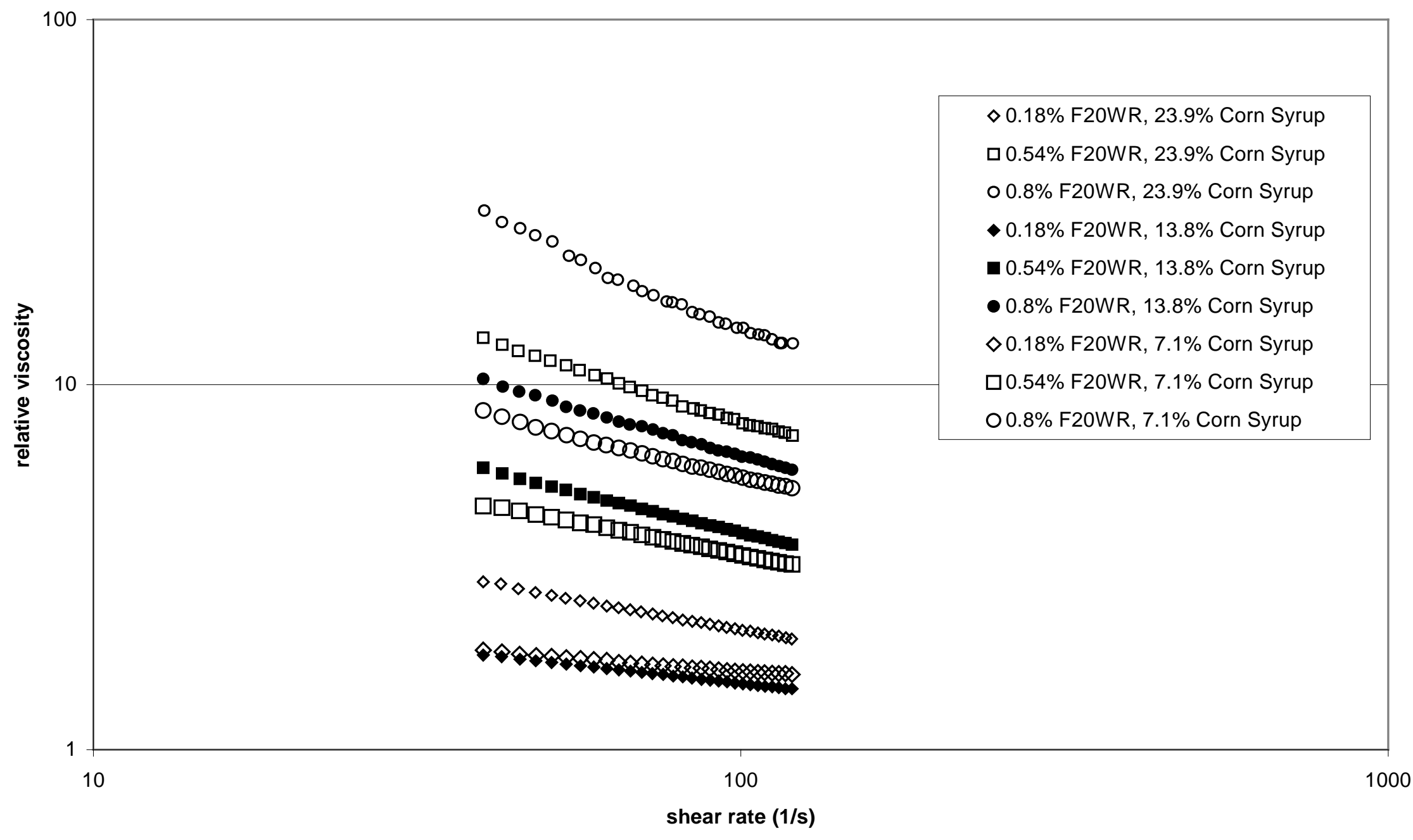

Figure B16 Relative visc. vs. shear rate for F20W 


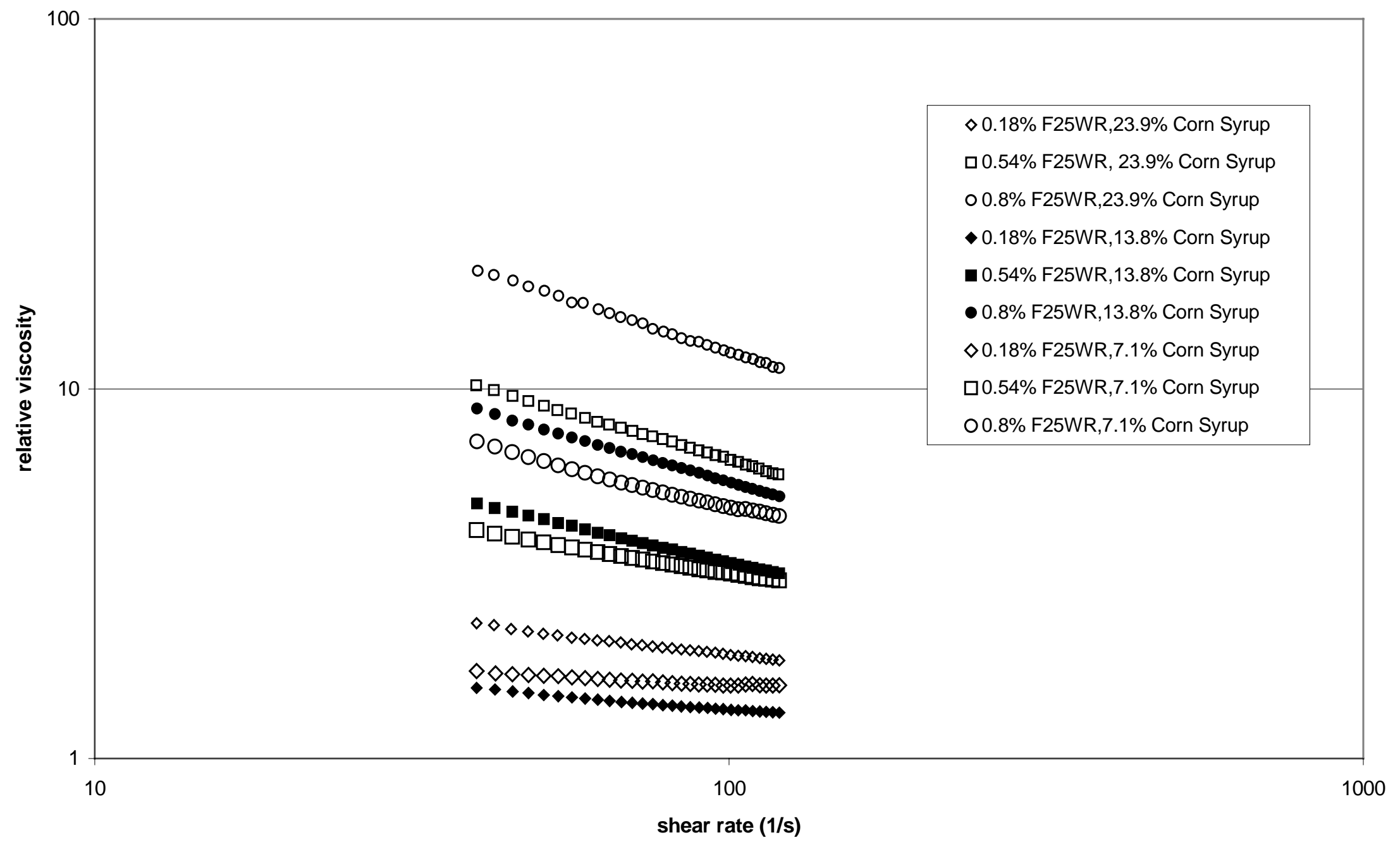

Figure B17 Relative visc. vs. shear rate for F25W 


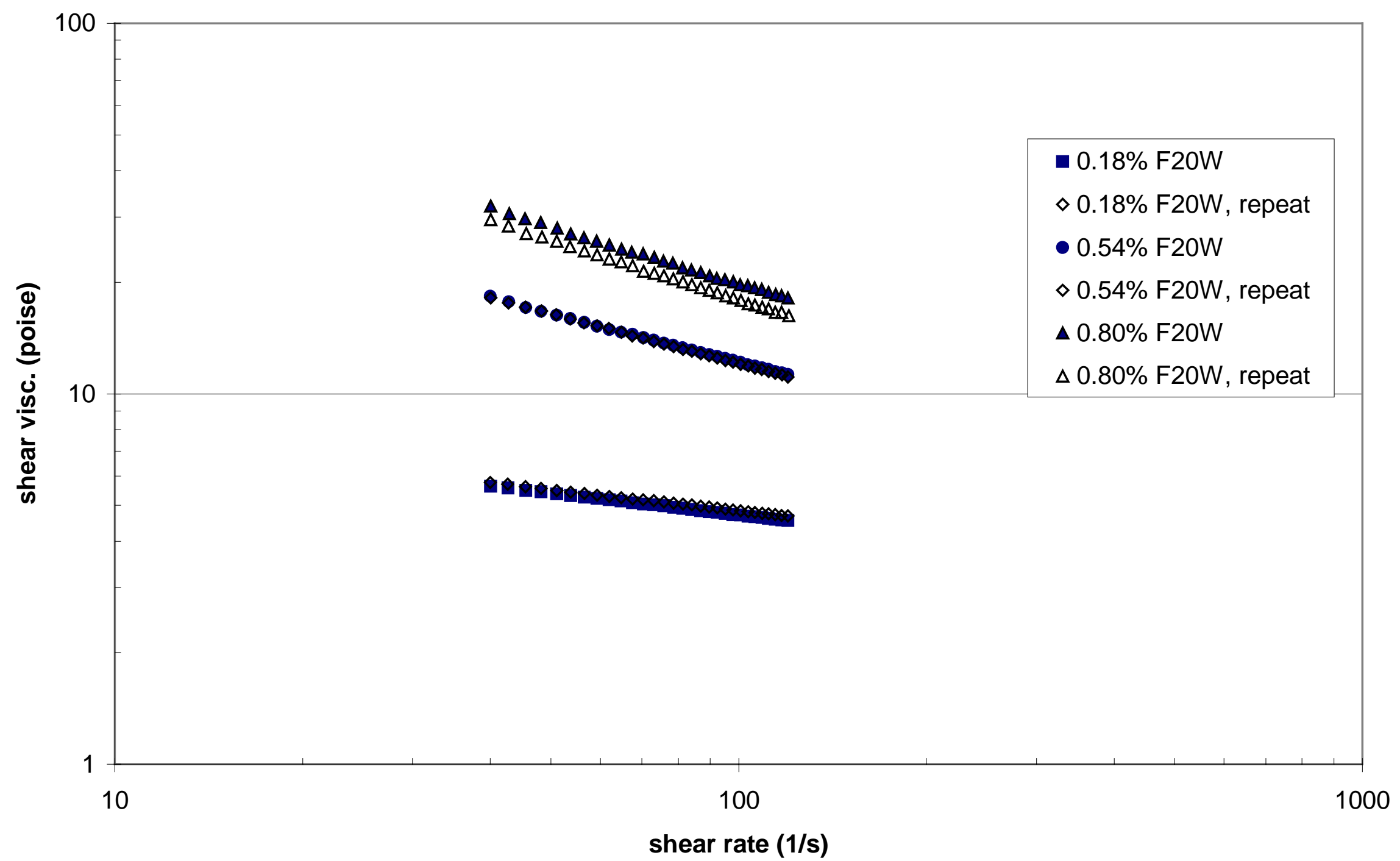

B18 Repetition of Results for F20W in Corn Syrup Solu. (13.8\% water) 
Table B1. Preparation of

suspensions

\begin{tabular}{|c|c|c|c|c|c|c|c|}
\hline Maste Solu.1.2\% Fibrid \& 7.1\% syrup & Syurp (g) & water $(\mathrm{g})$ & Fibrid (g),dry & \begin{tabular}{|l|} 
Fibrid (g), wet \\
\end{tabular} & Water in wet Fibrid (g) & Add. Water (g) & \\
\hline F10W,14.804\% in water (wet) & 1262.254 & 96.469358 & 16.5027129 & 111.4746883 & 94.97197544 & 1.497383007 & \\
\hline F20W, 20.081\% in water (wet) & 1272.771 & 97.273133 & 16.6402121 & 82.86545564 & 66.22524349 & 31.04788998 & \\
\hline F25W, 22.700\% in water (wet) & 1280.546 & 97.867348 & 16.7418625 & 73.75269834 & 57.01083581 & 40.85651187 & \\
\hline Goal solu. $150 \mathrm{~g}$ & Fibrid Conc. & syrup Conc. & Fibrid $(\mathrm{g})$ & Maste sol.(g) & syrup in Maste sol.(g) & Addi. syrup (g) & water $(\mathbf{g})$ \\
\hline $0.80 \%$ Fibrid \& $7.1 \%$ Corn syrup & $0.80 \%$ & $7.10 \%$ & 1.2 & 100 & 91.7852 & 46.45 & 3.55 \\
\hline $0.80 \%$ Fibrid \& $13.8 \%$ Corn syrup & $0.80 \%$ & $13.80 \%$ & 1.2 & 100 & 91.7852 & 36.4804 & 13.5196 \\
\hline $0.80 \%$ Fibrid \& 23.9\% Corn syrup & $0.80 \%$ & $23.90 \%$ & 1.2 & 100 & 91.7852 & 21.4516 & 28.5484 \\
\hline $0.54 \%$ Fibrid \& $7.1 \%$ Corn syrup & $0.54 \%$ & $7.10 \%$ & 0.81 & 67.5 & 61.95501 & 76.6425 & 5.8575 \\
\hline $0.54 \%$ Fibrid \& $13.8 \%$ Corn syrup & $0.54 \%$ & $13.80 \%$ & 0.81 & 67.5 & 61.95501 & 66.64677 & 15.8532 \\
\hline $0.54 \%$ Fibrid \& $23.9 \%$ Corn syrup & $0.54 \%$ & $23.90 \%$ & 0.81 & 67.5 & 61.95501 & 51.57858 & 30.9214 \\
\hline $0.18 \%$ Fibrid \& $7.1 \%$ Corn syrup & $0.18 \%$ & $7.10 \%$ & 0.27 & 22.5 & 20.65167 & 118.4475 & 9.0525 \\
\hline $0.18 \%$ Fibrid \& $13.8 \%$ Corn syrup & $0.18 \%$ & $13.80 \%$ & 0.27 & 22.5 & 20.65167 & 108.41559 & 19.0844 \\
\hline $0.18 \%$ Fibrid \& 23.9\% Corn syrup & $0.18 \%$ & $23.90 \%$ & 0.27 & 22.5 & 20.65167 & 93.29286 & 34.2071 \\
\hline
\end{tabular}


Appendix C Einstein Coefficient Data

Table C1 -- C13 


\begin{tabular}{|c|c|c|c|c|c|}
\hline \multicolumn{6}{|c|}{ Table C1 Preparation of F10W suspensions in corn syrup 1 (18\% H2O in corn syrup) } \\
\hline Master solu. Preparation & & & & & \\
\hline \multicolumn{6}{|l|}{ Goal Solu. $(150 \mathrm{~g})$} \\
\hline & & & & & \\
\hline Mast. Solu. & wet fibrid conc. & wet fibrid. (g) & syrup $(g)$ & Fibrid conc. $(\mathrm{g} / \mathrm{g})$ & Water Conc. $(\mathrm{g} / \mathrm{g})$ \\
\hline F10W (15.059\%) & 0.15059 & 1.5 & 148.5 & 0.0015059 & 0.0084941 \\
\hline F10W (15.059\%) & 0.15059 & 1.5 & 148.5 & 0.0015059 & 0.0084941 \\
\hline F10W (15.059\%) & 0.15059 & 1.5 & 148.5 & 0.0015059 & 0.0084941 \\
\hline F10W (15.059\%) & 0.15059 & 1.5 & 148.5 & 0.0015059 & 0.0084941 \\
\hline F10W (15.059\%) & 0.15059 & 1.5 & 148.5 & 0.0015059 & 0.0084941 \\
\hline \multirow{2}{*}{\multicolumn{6}{|c|}{\begin{tabular}{|l|l} 
Measure solu. Preparation & $18 \%$ \\
Goal solu. $(150 \mathrm{~g})$ & \\
\end{tabular}}} \\
\hline & & & & & \\
\hline \multicolumn{6}{|c|}{ Fibrid pom master solu. (a) } \\
\hline F10W & 0.0015059 & 0 & 0.000 & 123.000 & 27.000 \\
\hline F10W & 0.0015059 & 100 & 9.961 & 113.126 & 26.913 \\
\hline F10W & 0.0015059 & 200 & 19.922 & 103.253 & 26.825 \\
\hline F10W & 0.0015059 & 300 & 29.882 & 93.379 & 26.738 \\
\hline \multirow[t]{2}{*}{ F10W } & 0.0015059 & 400 & 39.843 & 83.506 & 26.651 \\
\hline & & Total: & 99.608 & 393.265 & 107.127 \\
\hline
\end{tabular}




\begin{tabular}{|c|c|c|c|c|c|}
\hline \multicolumn{6}{|c|}{ Table C2 Preparation of F20W suspensions in corn syrup 1 (18\% H2O in corn syrup) } \\
\hline Master solu. Preparation & & & & & \\
\hline \multicolumn{6}{|l|}{ Goal Solu. $(150 \mathrm{~g})$} \\
\hline Mast. Solu. & wet fibrid conc. & $\begin{array}{r}\text { wet fibrid. } \\
(\mathrm{g})\end{array}$ & syrup $(g)$ & Fibrid conc. $(\mathrm{g} / \mathrm{g})$ & Water Conc. $(\mathrm{g} / \mathrm{g})$ \\
\hline F20W (22.608\%) & 0.22608 & 1 & 149 & 0.0015072 & 0.005159467 \\
\hline F20W (22.608\%) & 0.22608 & 1 & 149 & 0.0015072 & 0.005159467 \\
\hline F20W (22.608\%) & 0.22608 & 1 & 149 & 0.0015072 & 0.005159467 \\
\hline F20W (22.608\%) & 0.22608 & 1 & 149 & 0.0015072 & 0.005159467 \\
\hline F20W (22.608\%) & 0.22608 & 1 & 149 & 0.0015072 & 0.005159467 \\
\hline Measure solu. Preparation & $18 \% \mathrm{H} 2 \mathrm{O}$ in corn syrup & & & & \\
\hline \multicolumn{6}{|l|}{ Goal solu. $(150 \mathrm{~g})$} \\
\hline Fibrid & master solu. conc. & Fibrid ppm & master solu. (g) & syrup (g) & water $(\mathrm{g})$ \\
\hline F20W & 0.0015072 & 0 & 0.000 & 123.000 & 27.000 \\
\hline F20W & 0.0015072 & 100 & 9.952 & 113.102 & 26.946 \\
\hline F20W & 0.0015072 & 200 & 19.904 & 103.204 & 26.892 \\
\hline F20W & 0.0015072 & 300 & 29.857 & 93.305 & 26.838 \\
\hline \multirow[t]{2}{*}{ F20W } & 0.0015072 & 400 & 39.809 & 83.407 & 26.784 \\
\hline & & Total: & 99.522 & 393.018 & 107.460 \\
\hline
\end{tabular}




\begin{tabular}{|l|r|r|r|r|r|}
\hline \multicolumn{5}{|c|}{ Table C3 Preparation of F25W suspensions in corn syrup 1 (18\% H2O in corn syrup) } \\
\hline Master solu. Preparation & & & & & \\
\hline Goal Solu. (150 g) & & & & & \\
\hline & & & & & \\
\hline Mast. Solu. & wet fibrid conc. & wet fibrid. (g) & syrup (g) & Fibrid conc. (g/g) & Water Conc. (g/g) \\
\hline F25W (25.444\%) & 0.25444 & 1 & 149 & 0.001696267 & 0.0049704 \\
\hline F25W (25.444\%) & 0.25444 & 1 & 149 & 0.001696267 & 0.0049704 \\
\hline F25W (25.444\%) & 0.25444 & 1 & 149 & 0.001696267 & 0.0049704 \\
\hline F25W (25.444\%) & 0.25444 & 1 & 149 & 0.001696267 & 0.0049704 \\
\hline F25W (25.444\%) & 0.25444 & 1 & 149 & 0.001696267 & 0.0049704 \\
\hline & & & & & \\
\hline Measure solu. Preparation & $18 \%$ H2O in corn syrup & & & & \\
\hline Goal solu. (150 g) & & & & & \\
\hline & & & & & \\
\hline Fibrid & master solu. conc. & Fibrid ppm & master solu. (g) & syrup (g) & water (g) \\
\hline F25W & 0.001696267 & 0 & $\mathbf{0 . 0 0 0}$ & $\mathbf{1 2 3 . 0 0 0}$ & $\mathbf{2 7 . 0 0 0}$ \\
\hline F25W & 0.001696267 & 100 & $\mathbf{8 . 8 4 3}$ & $\mathbf{1 1 4 . 2 0 4}$ & $\mathbf{2 6 . 9 5 3}$ \\
\hline F25W & 0.001696267 & 200 & $\mathbf{1 7 . 6 8 6}$ & $\mathbf{1 0 5 . 4 0 7}$ & $\mathbf{2 6 . 9 0 7}$ \\
\hline F25W & 0.001696267 & 300 & $\mathbf{2 6 . 5 2 9}$ & $\mathbf{9 6 . 6 1 1}$ & $\mathbf{2 6 . 8 6 0}$ \\
\hline F25W & 0.001696267 & 400 & $\mathbf{3 5 . 3 7 2}$ & $\mathbf{8 7 . 8 1 5}$ & $\mathbf{2 6 . 8 1 3}$ \\
\hline & & & $\mathbf{8 8 . 4 2 9}$ & $\mathbf{4 0 4 . 0 3 7}$ & $\mathbf{1 0 7 . 5 3 3}$ \\
\hline
\end{tabular}


Table C4 Measurement results for glass bead in hydraulic oil suspension

\begin{tabular}{|c|c|c|c|c|c|c|c|}
\hline & & & & density & $\mathrm{g} / \mathrm{ml}$ & \\
\hline & & drain time & & HO & 0.881 & \\
\hline & Hydraulic Oil (s) & 449.34 & & GB & 2.56 & & \\
\hline & & & & & & & \\
\hline Solu. Conc. & Volume Fraction & GB (11u,s) & Rel. Vis. & error & GB (4u, s) & Rel. Vis. & error \\
\hline 0.013 & 0.004512301 & 452.4 & 1.007 & 0.002 & 453.0 & 1.008 & 0.002 \\
\hline 0.026 & 0.009102882 & 457.9 & 1.019 & 0.002 & 457.8 & 1.019 & 0.002 \\
\hline 0.039 & 0.013773798 & 461.6 & 1.027 & 0.002 & 462.0 & 1.028 & 0.002 \\
\hline 0.052 & 0.018527176 & 466.3 & 1.038 & 0.002 & 466.9 & 1.039 & 0.002 \\
\hline error: $+-0.5 \mathrm{sec}$ & for drain time(s) & & & & & & \\
\hline
\end{tabular}


Table C5 Measurement results for hollow glass bead in hydraulic oil suspension

\begin{tabular}{|c|c|c|c|c|c|}
\hline & & & density & $\mathrm{g} / \mathrm{ml}$ & \\
\hline & & drain time & HO & 0.881 & \\
\hline & Hydraulic Oil (s) & 449.34 & HGB & 1.128 & \\
\hline Solu. Conc. & Volume Fraction & HGB (s) & Rel. Vis. & error & Rel. Vis. (Theory) \\
\hline 0.006 & 0.004692335 & 454.26 & 1.011 & 0.002 & 1.012 \\
\hline 0.012 & 0.009397033 & 459.59 & 1.023 & 0.002 & 1.023 \\
\hline 0.018 & 0.014114141 & 465.21 & 1.035 & 0.002 & 1.035 \\
\hline 0.024 & 0.018843711 & 470.54 & 1.047 & 0.002 & 1.047 \\
\hline Error: $+-0.5 \mathrm{sec}$ & for drain time(s) & & & & \\
\hline
\end{tabular}


Table C6 Measurement results for hollow glass bead in corn syrup suspension

\begin{tabular}{|c|c|c|c|c|c|}
\hline & & & density & $\mathrm{g} / \mathrm{ml}$ & \\
\hline & & drain time & Corn Syrup & 1.382 & \\
\hline & Corn Syrup (s) & 293.2 & HGB & 1.128 & \\
\hline Solu. Conc. & Volume Fraction & HGB (s) & Rel. Vis. & error & Rel. Vis. (Theory) \\
\hline 0.012 & 0.014662508 & 324.70 & 1.107 & 0.003 & 1.037 \\
\hline 0.024 & 0.029246201 & 352.14 & 1.201 & 0.003 & 1.073 \\
\hline 0.036 & 0.043751715 & 380.87 & 1.299 & 0.003 & 1.109 \\
\hline 0.048 & 0.058179675 & 406.80 & 1.387 & 0.003 & 1.145 \\
\hline error: $+-0.5 \mathrm{sec}$ & for drain time(s) & & & & \\
\hline
\end{tabular}


Table C7 Measurement results for hollow glass bead in corn syrup 1 (18\% water) suspension

\begin{tabular}{|c|c|c|c|c|c|}
\hline & & drain time & density & $\mathrm{g} / \mathrm{ml}$ & \\
\hline & Corn Syrup (s) & 293.2 & Corn Syrup & 1.382 & \\
\hline & $18 \%$ corn syrup (s) & 379 & & 1.313 & \\
\hline & & & HGB & 1.128 & \\
\hline Solu. Conc. & Volume Fraction & HGB (s) & Rel. Vis. & error & Rel. Vis. (Theory) \\
\hline 0.012 & 0.013940649 & 392.53 & 1.036 & 0.003 & 1.035 \\
\hline 0.024 & 0.02782664 & 405.86 & 1.071 & 0.003 & 1.070 \\
\hline 0.036 & 0.041658294 & 419.23 & 1.106 & 0.003 & 1.104 \\
\hline 0.048 & 0.05543593 & 432.51 & 1.141 & 0.002 & 1.139 \\
\hline error: $+-0.5 \mathrm{sec}$ & for drain time(s) & & & & \\
\hline
\end{tabular}


Table C8 Measurement results for hollow glass bead in corn syrup 2 (20\% water) suspension

\begin{tabular}{|c|c|c|c|c|c|c|}
\hline & & drain time & density & $\mathrm{g} / \mathrm{ml}$ & & \\
\hline & Corn Syrup (s) & & Corn Syrup & 1.382 & & \\
\hline & $20 \%$ corn syrup (s) & 292.4 & & 1.306 & & \\
\hline & & & HGB & 1.128 & & \\
\hline Solu. Conc. & Volume Fraction & HGB (s) & Rel. Vis. & error & Rel. Vis. (Theory) & Theory (s) \\
\hline 0.012 & 0.013867358 & 303.20 & 1.037 & 0.003 & 1.035 & 302.537 \\
\hline 0.024 & 0.027682394 & 313.58 & 1.072 & 0.003 & 1.069 & 312.636 \\
\hline 0.036 & 0.041445406 & 321.90 & 1.101 & 0.003 & 1.104 & 322.697 \\
\hline 0.048 & 0.055156686 & 333.83 & 1.142 & 0.003 & 1.138 & 332.720 \\
\hline error: $+-0.5 \mathrm{sec}$ & for drain time(s) & & & & & \\
\hline
\end{tabular}




\begin{tabular}{|c|c|c|c|c|}
\hline \multicolumn{2}{|c|}{ Table C9 Measurement results for fibrid in corn syrup 1 suspension } & drain time & density & $\mathrm{g} / \mathrm{ml}$ \\
\hline & Corn Syrup (s) & 293.2 & Corn Syrup & 1.382 \\
\hline & $18 \%$ corn syrup (s) & 376.23 & & 1.313 \\
\hline & & & Fibrid & 1.38 \\
\hline & & & & \\
\hline Solu. Conc.(ppm) & Volume Fraction & F10W (s) & Rel. Vis. & error \\
\hline 91.96 & 8.74957E-05 & 391.68 & 1.041 & 0.003 \\
\hline 183.92 & 0.000174992 & 407.66 & 1.084 & 0.003 \\
\hline 275.87 & 0.00026248 & 425.71 & 1.132 & 0.003 \\
\hline 367.83 & 0.000349978 & 445.84 & 1.185 & 0.002 \\
\hline error: + - $0.5 \mathrm{sec}$ & for drain time(s) & & & \\
\hline Solu. Conc.(ppm) & Volume Fraction & F20W (s) & Rel. Vis. & error \\
\hline 100 & 9.51454E-05 & 390.01 & 1.037 & 0.003 \\
\hline 200 & 0.000190292 & 405.74 & 1.078 & 0.003 \\
\hline 300 & 0.000285439 & 421.21 & 1.120 & 0.003 \\
\hline 400 & 0.000380587 & 443.44 & 1.179 & 0.002 \\
\hline error: + - $0.5 \mathrm{sec}$ & for drain time(s) & & & \\
\hline Solu. Conc.(ppm) & Volume Fraction & F25W (s) & Rel. Vis. & error \\
\hline 100 & $9.51454 \mathrm{E}-05$ & 383.70 & 1.020 & 0.003 \\
\hline 200 & 0.000190292 & 391.48 & 1.041 & 0.003 \\
\hline 300 & 0.000285439 & 401.79 & 1.068 & 0.003 \\
\hline 400 & 0.000380587 & 413.59 & 1.099 & 0.003 \\
\hline error: + - $0.5 \mathrm{sec}$ & for drain time(s) & & & \\
\hline
\end{tabular}




\begin{tabular}{|c|c|c|c|c|}
\hline \multicolumn{2}{|c|}{ Table C10 Measurement results for fibrid in corn syrup 2 suspension } & drain time & density & $\mathrm{g} / \mathrm{ml}$ \\
\hline & Corn Syrup (s) & 293.2 & Corn Syrup & 1.382 \\
\hline & $20 \%$ corn syrup (s) & 289 & & 1.306 \\
\hline & & & Fibrid & 1.38 \\
\hline Solu. Conc.(ppm) & Volume Fraction & $\mathrm{F} 10 \mathrm{~W}(\mathrm{~s})$ & Rel. Vis. & error \\
\hline 101.0738976 & 9.56545E-05 & 307.47 & 1.064 & 0.003 \\
\hline 202.1477952 & 0.00019131 & 328.99 & 1.138 & 0.003 \\
\hline 303.2216927 & 0.000286967 & 341.87 & 1.183 & 0.003 \\
\hline \begin{tabular}{|l|}
404.2955903 \\
\end{tabular} & 0.000382624 & 372.33 & 1.288 & 0.003 \\
\hline error: + - $0.5 \mathrm{sec}$ & for drain time(s) & & & \\
\hline Solu. Conc.(ppm) & Volume Fraction & F20W (s) & Rel. Vis. & error \\
\hline 103.431238 & 9.78855E-05 & 301.52 & 1.043 & 0.003 \\
\hline 206.862476 & 0.000195772 & 320.93 & 1.110 & 0.003 \\
\hline 310.293714 & 0.00029366 & 330.20 & 1.143 & 0.003 \\
\hline 413.724952 & 0.000391548 & 352.08 & 1.218 & 0.003 \\
\hline error: + - $0.5 \mathrm{sec}$ & for drain time(s) & & & \\
\hline Solu. Conc.(ppm) & Volume Fraction & F25W (s) & Rel. Vis. & error \\
\hline \begin{tabular}{|l|}
102.1232189 \\
\end{tabular} & 9.66476E-05 & 300.04 & 1.038 & 0.003 \\
\hline 204.2464379 & 0.000193296 & 309.36 & 1.070 & 0.003 \\
\hline 306.3696568 & 0.000289946 & 319.20 & 1.104 & 0.003 \\
\hline 408.4928758 & 0.000386597 & 330.20 & 1.143 & 0.003 \\
\hline error: + - $0.5 \mathrm{sec}$ & for drain time(s) & & & \\
\hline
\end{tabular}




\begin{tabular}{|c|c|c|c|c|}
\hline \multicolumn{2}{|c|}{ Table C11 Final Measurement results for fibrid in corn syrup 2 suspension } & drain time & density & $\mathrm{g} / \mathrm{ml}$ \\
\hline & Corn Syrup (s) & 293.2 & Corn Syrup & 1.382 \\
\hline & $20 \%$ corn syrup (s) & 289 & & 1.306 \\
\hline & & & Fibrid & 1.38 \\
\hline Solu. Conc.(ppm) & Volume Fraction & $\mathrm{F} 10 \mathrm{~W}(\mathrm{~s})$ & Rel. Vis. & error \\
\hline 101.0738976 & $9.56545 \mathrm{E}-05$ & 300.14 & 1.039 & 0.003 \\
\hline 202.1477952 & 0.00019131 & 313.25 & 1.084 & 0.003 \\
\hline 303.2216927 & 0.000286967 & 324.90 & 1.124 & 0.003 \\
\hline 404.2955903 & 0.000382624 & 337.60 & 1.168 & 0.003 \\
\hline error: + - $0.5 \mathrm{sec}$ & for drain time(s) & & & \\
\hline Solu. Conc.(ppm) & Volume Fraction & F20W (s) & Rel. Vis. & error \\
\hline 103.431238 & $9.78855 \mathrm{E}-05$ & 299.58 & 1.037 & 0.003 \\
\hline 206.862476 & 0.000195772 & 312.13 & 1.080 & 0.003 \\
\hline 310.293714 & 0.00029366 & 324.50 & 1.123 & 0.003 \\
\hline 413.724952 & 0.000391548 & 336.61 & 1.165 & 0.003 \\
\hline error: + - $0.5 \mathrm{sec}$ & for drain time(s) & & & \\
\hline Solu. Conc.(ppm) & Volume Fraction & F25W (s) & Rel. Vis. & error \\
\hline 102.1232189 & $9.66476 \mathrm{E}-05$ & 298.33 & 1.032 & 0.003 \\
\hline \begin{tabular}{|l|}
204.2464379 \\
\end{tabular} & 0.000193296 & 310.58 & 1.075 & 0.003 \\
\hline 306.3696568 & 0.000289946 & 320.03 & 1.107 & 0.003 \\
\hline 408.4928758 & 0.000386597 & 329.89 & 1.141 & 0.003 \\
\hline error: + - $0.5 \mathrm{sec}$ & for drain time(s) & & & \\
\hline
\end{tabular}


Table C12 check the final measurement results for fibrid in corn syrup 2 suspension

\begin{tabular}{|l|r|r|r|r|r|}
\hline drain time (s) & corrected procedure & repeat & \multicolumn{2}{l|}{ previous procedure } & repeat \\
\hline 20\% water in corn syrup & (step 9) & & & 289 & 288.7 \\
\hline F10W 100ppm & 300.14 & & 307.47 & 307.2 \\
\hline F10W 200ppm & 313.25 & & 328.99 & \\
\hline F10W 300ppm & 324.9 & & & 341.87 & \\
\hline F10W 400ppm & 337.6 & 337.3 & 372.33 & \\
\hline F20W 100ppm & 299.58 & & & 301.52 & \\
\hline F20W 200ppm & 312.13 & & & 320.93 & 302 \\
\hline F20W 300ppm & 324.5 & & & 330.2 & \\
\hline F20W 400ppm & 336.61 & 336.2 & & 352.08 & \\
\hline F25W 100ppm & 298.33 & & & 300.04 & \\
\hline F25W 200ppm & 310.58 & & & 309.36 & 300.1 \\
\hline F25W 300ppm & 320.03 & & & 319.2 & \\
\hline F25W 400ppm & 329.89 & 329.8 & & 330.2 & \\
\hline
\end{tabular}




\begin{tabular}{|c|c|c|c|c|}
\hline \multicolumn{2}{|c|}{ Table C13 Final Measurement results for fibrid in corn syrup 1 suspension } & drain time & density & $\mathrm{g} / \mathrm{ml}$ \\
\hline & Corn Syrup (s) & 293.2 & Corn Syrup & 1.382 \\
\hline & $18 \%$ corn syrup $(\mathrm{s})$ & 379.66 & & 1.313 \\
\hline & & & Fibrid & 1.38 \\
\hline Solu. Conc.(ppm) & Volume Fraction & $\mathrm{F} 10 \mathrm{~W}(\mathrm{~s})$ & Rel. Vis. & error \\
\hline 100 & 9.51454E-05 & 396.80 & 1.045 & 0.003 \\
\hline 200 & 0.000190292 & 413.72 & 1.090 & 0.003 \\
\hline 300 & 0.000285439 & 428.99 & 1.130 & 0.002 \\
\hline 400 & 0.000380587 & 448.65 & 1.182 & 0.002 \\
\hline error: + - $0.5 \mathrm{sec}$ & for drain time(s) & & & \\
\hline Solu. Conc.(ppm) & Volume Fraction & F20W (s) & Rel. Visc. & error \\
\hline 100 & 9.51454E-05 & 393.85 & 1.037 & 0.003 \\
\hline 200 & 0.000190292 & 410.28 & 1.081 & 0.003 \\
\hline 300 & 0.000285439 & 426.49 & 1.123 & 0.002 \\
\hline 400 & 0.000380587 & 445.60 & 1.174 & 0.002 \\
\hline error: + - $0.5 \mathrm{sec}$ & for drain time(s) & & & \\
\hline Solu. Conc.(ppm) & Volume Fraction & F25W (s) & Rel. Visc. & error \\
\hline 100 & 9.51454E-05 & 391.27 & 1.031 & 0.003 \\
\hline 200 & 0.000190292 & 405.56 & 1.068 & 0.003 \\
\hline 300 & 0.000285439 & 419.84 & 1.106 & 0.003 \\
\hline 400 & 0.000380587 & 435.89 & 1.148 & 0.002 \\
\hline error: + - $0.5 \mathrm{sec}$ & for drain time(s) & & & \\
\hline
\end{tabular}


Appendix D Extensional Viscosity Data

Figure D1 - D6

Table D1 -- D3 


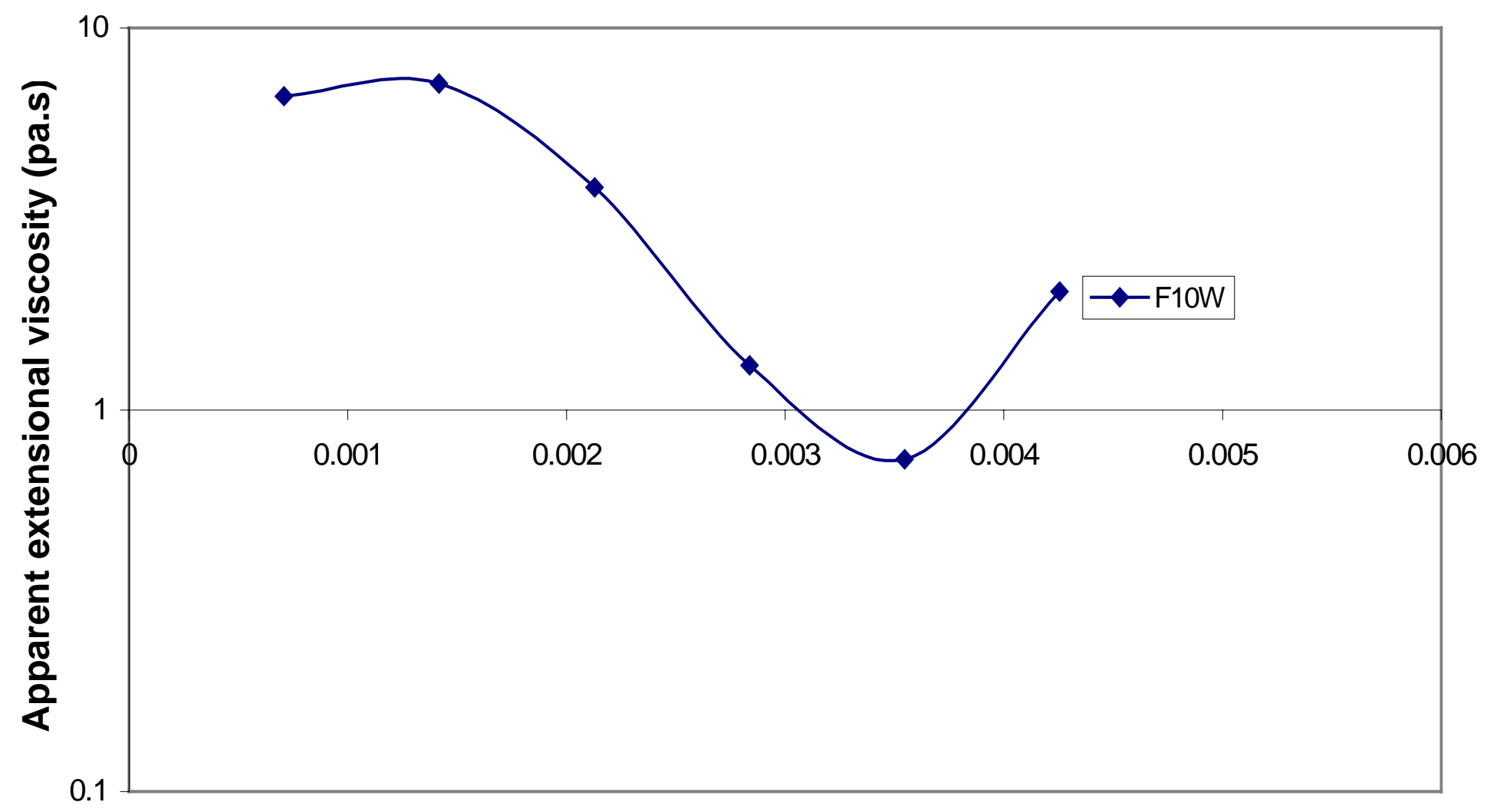

Distance from upper capillary (m)

Figure D1 Apparent extensional viscosity vs. distance from the exit of upper capillary for F10W 200ppm in $25 \%$ corn syrup 


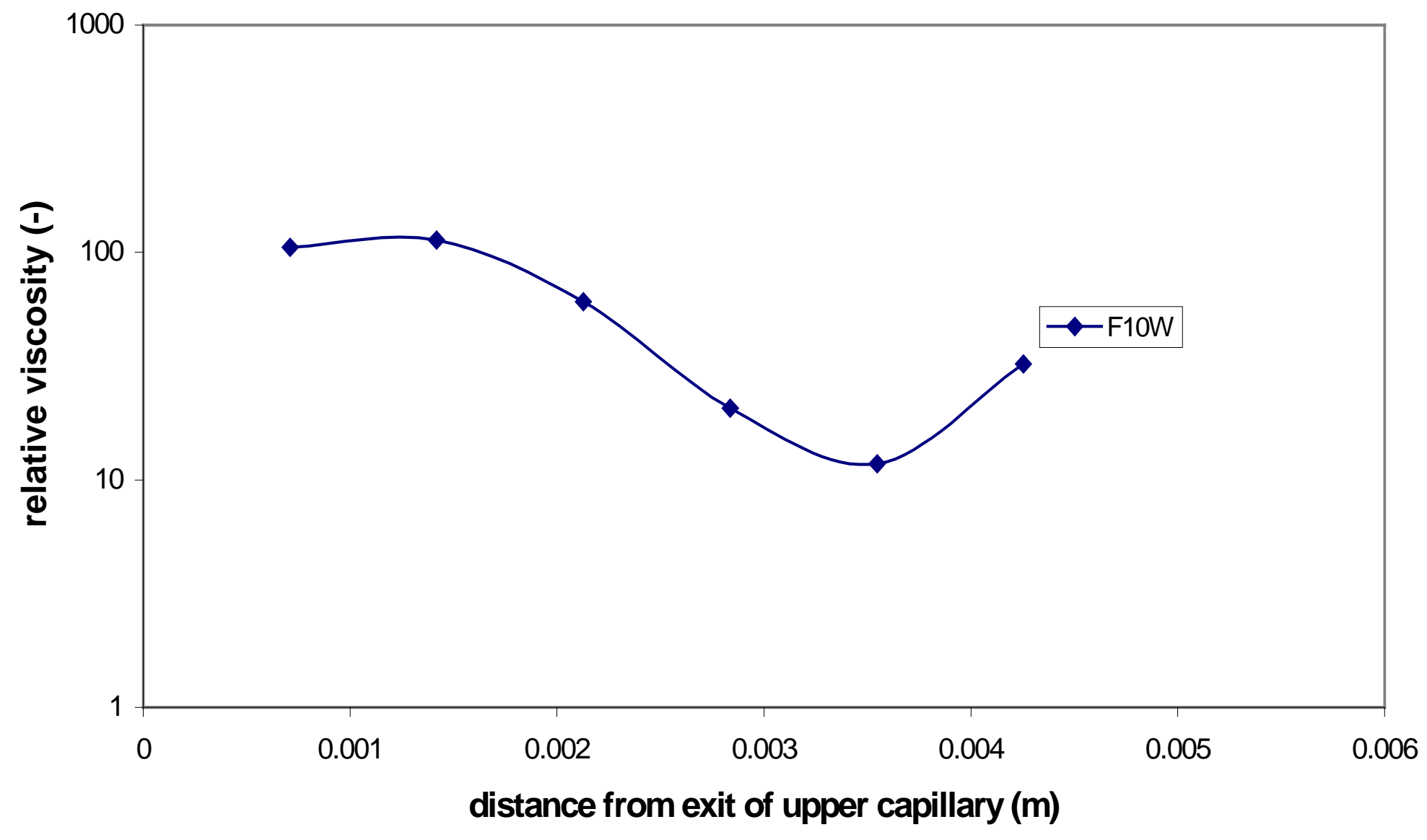

Figure D2 Relative viscosity vs. distance from exit of upper capillary for F10W 200ppm 25\% corn syrup 


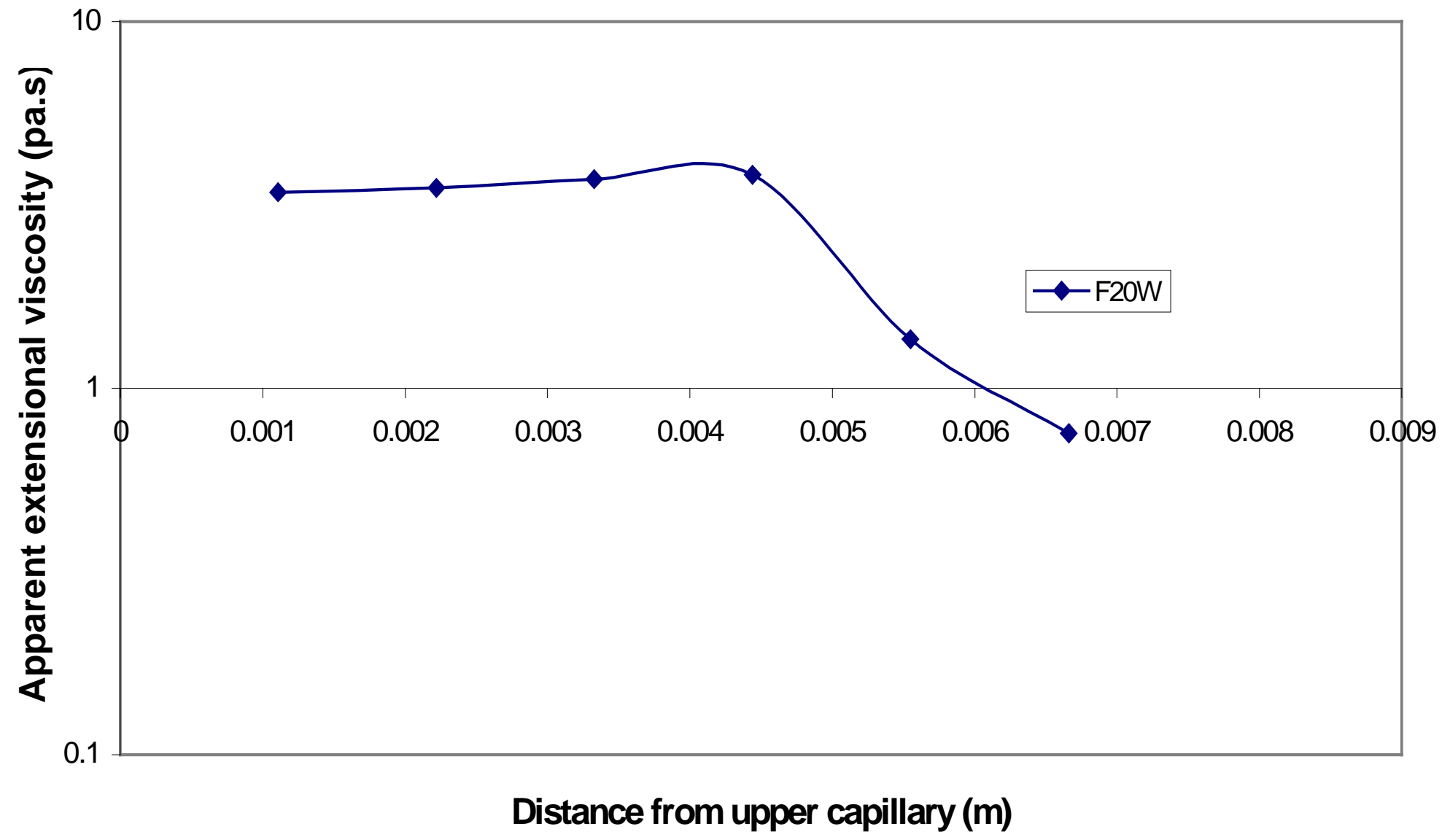

Figure D3 Apparent extensional viscosity vs. distance from the exit of upper capillary for F20W 200ppm in 25\% corn syrup 


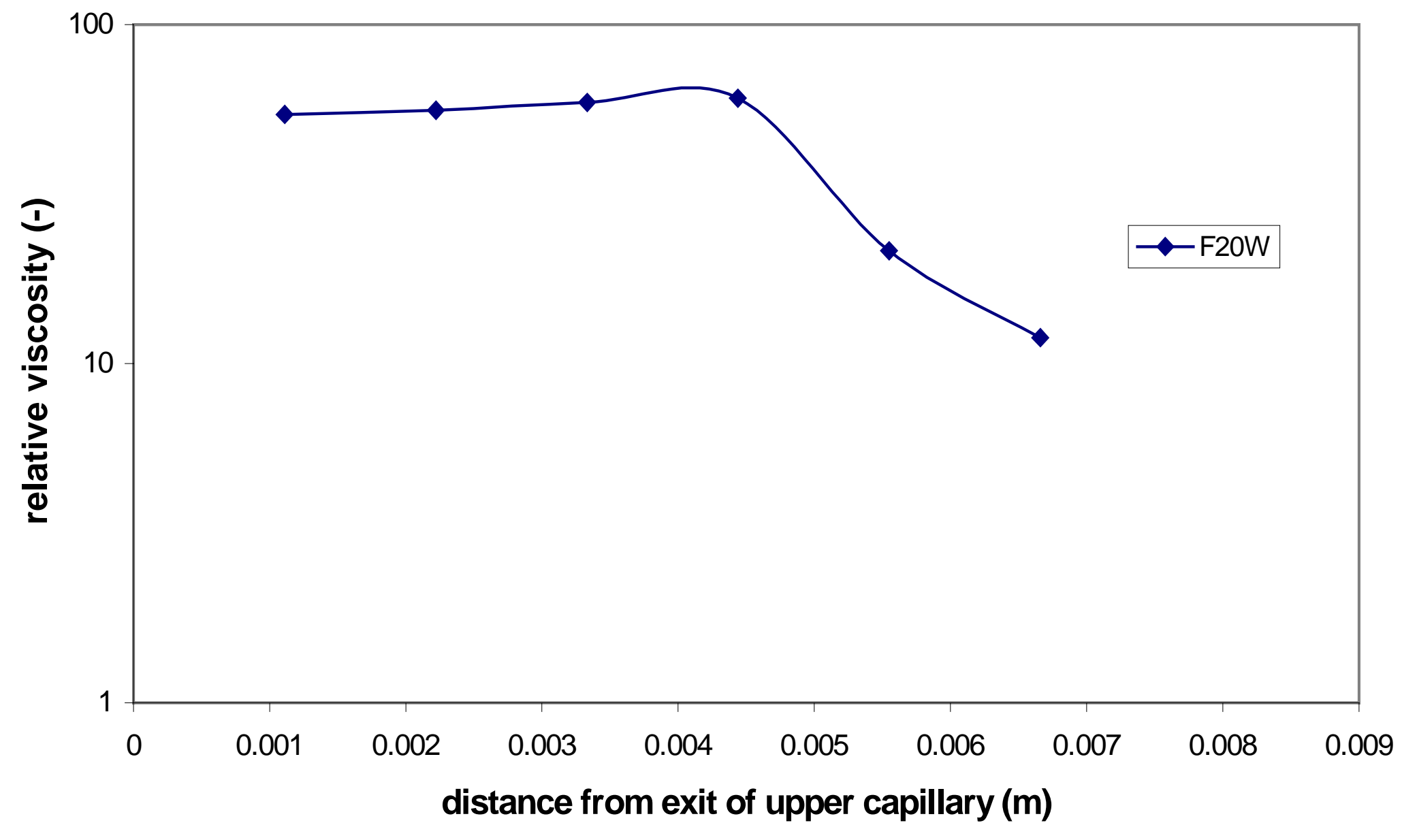

Figure D4 Relative viscosity vs. distance from exit of upper capillary for F20W 200ppm 25\% corn syrup 


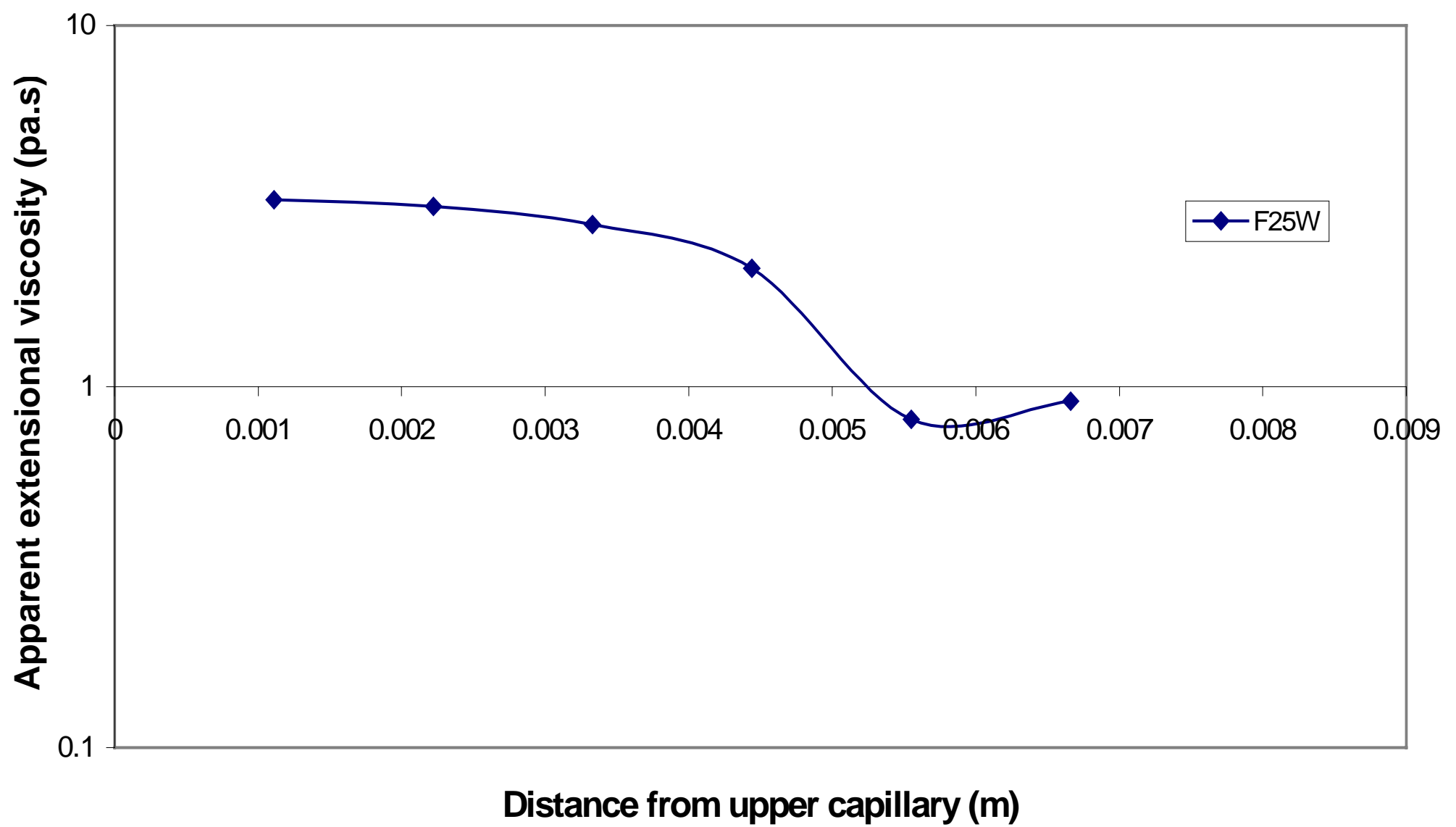

Figure D5 Apparent extensional viscosity vs. distance from the exit of upper capillary for F25W 200ppm in $25 \%$ corn syrup 


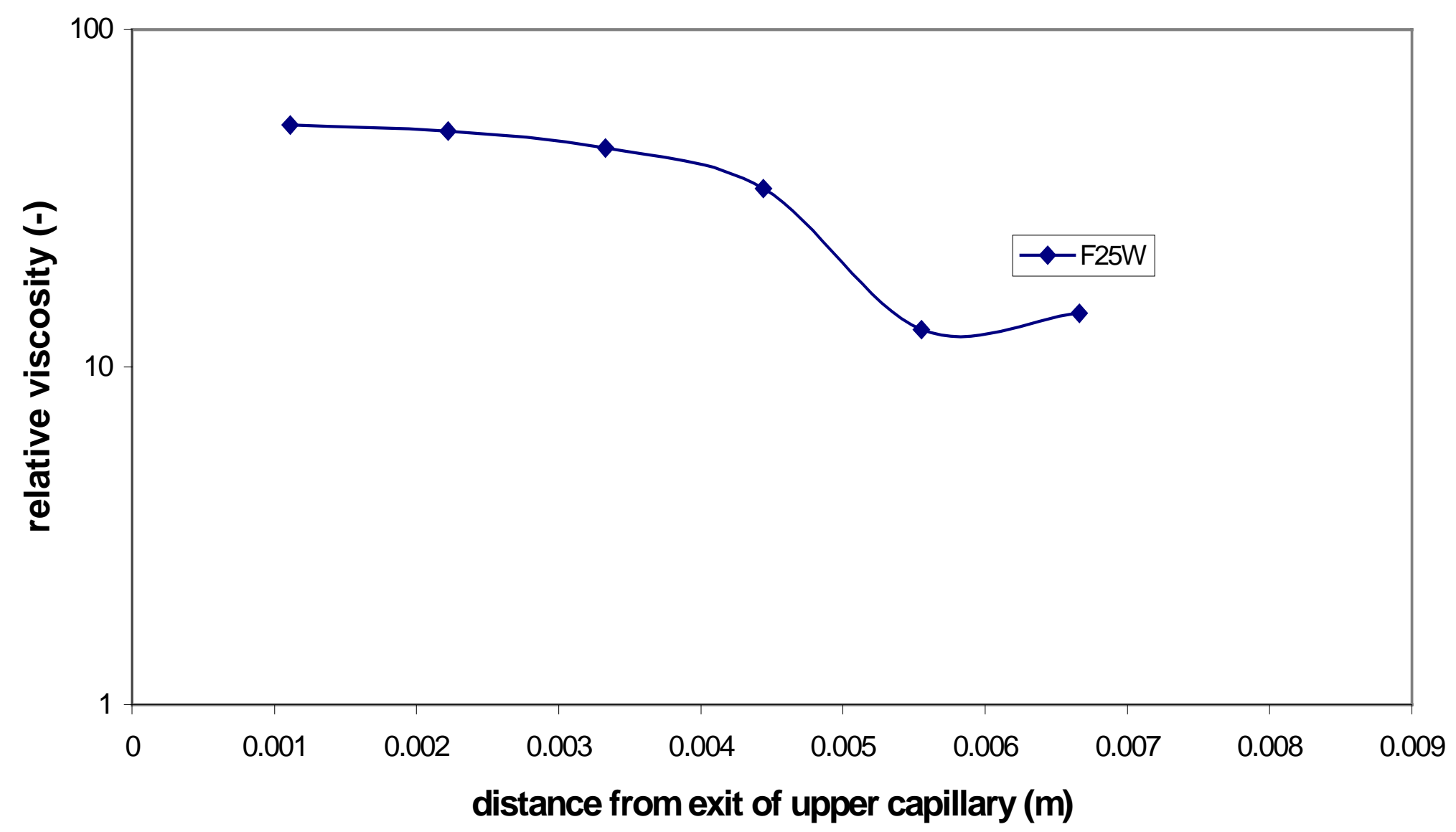

Figure D6 Relative viscosity vs. distance from exit of upper capillary for F25W 200ppm 25\% corn syrup 
Table D1 Calculation detail for 200 ppm F10W suspension in corn syrup solution (25\% water in weight)

\begin{tabular}{|c|c|c|c|c|c|c|c|}
\hline & Thin Wall Tube & Gauge & 10-12 for 200ppm & $\mathrm{F} 10 \mathrm{~W}$ at $25 \%$ & corn syrup & & \\
\hline & & & & density $(\mathrm{g} / \mathrm{ml})$ & 1.262 & & \\
\hline & & & shear viscosity & (pa.s) & 0.063 & & \\
\hline & & & suface tension coe. & $(\mathrm{N} / \mathrm{m})$ & 0.07 & & \\
\hline & flow rate & measure & & & & & \\
\hline & & & & & & read. diff. & pre. Diff. \\
\hline & flow volume $(\mathrm{ml})$ & time (s) & flow rate $(\mathrm{ml} / \mathrm{s})$ & flow rate $(\mathrm{m} 3 / \mathrm{s})$ & & $(\mathrm{mmH} 2 \mathrm{O})$ & (pa) \\
\hline 1 & 9.8 & 11.88 & 0.824915825 & $8.24916 \mathrm{E}-07$ & & 4 & 42.61824 \\
\hline 2 & 9.8 & 11.8 & 0.830508475 & $8.30508 \mathrm{E}-07$ & & (degree 5 & for $U$ tube) \\
\hline 3 & 10 & 12.16 & 0.822368421 & $8.22368 \mathrm{E}-07$ & & & \\
\hline ave. & & & & $8.25931 \mathrm{E}-07$ & & & \\
\hline & $\mathrm{F}(0)=(\mathrm{P} 0-\mathrm{P} 1)^{\star} \mathrm{A} 0 \quad(\mathrm{~N})$ & 0.00038785 & & & & & \\
\hline & Sec. term in $F(I)$ & $1.3812 \mathrm{E}-07$ & $\left(16^{\star} \mathrm{Q}^{\wedge} 2 /\left(3^{*}\right.\right.$ pai $\left.\left.^{*} \mathrm{~d}^{\wedge} 2\right)\right)$ & & & & \\
\hline & & & & & & & \\
\hline & Upp. Tub. OD (m) & 0.0034036 & ID (m) & 0.0028956 & & & \\
\hline & Dist. From exit of Upp. Capilary & & Filament diameter & & Cross sec. area & Velocity (v) & $\mathrm{dv} / \mathrm{dz}$ \\
\hline & $(-)$ & $(\mathrm{m})$ & $(-)(z)$ & $(\mathrm{m})$ & $\left(\mathrm{m}^{\wedge} 2\right)$ & $(\mathrm{m} / \mathrm{s})$ & $(1 / \mathrm{s})$ \\
\hline & 0 & 0 & 48 & 0.003404 & $9.1006 \mathrm{E}-06$ & 0.0907557 & \\
\hline & 10 & 0.00070917 & 45 & 0.00319125 & 7.99857E-06 & 0.1032598 & 12.162922 \\
\hline & 20 & 0.00141833 & 44 & 0.003120333 & $7.64703 \mathrm{E}-06$ & 0.1080067 & 10.771962 \\
\hline & 30 & 0.0021275 & 42 & 0.0029785 & $6.96765 \mathrm{E}-06$ & 0.118538 & 20.777412 \\
\hline & 40 & 0.00283667 & 39 & 0.00276575 & $6.00782 \mathrm{E}-06$ & 0.137476 & 69.834738 \\
\hline & 50 & 0.00354583 & 31 & 0.002198417 & 3.79587E-06 & 0.217587 & 237.3744 \\
\hline & 60 & 0.004255 & 21 & 0.00148925 & $1.74191 \mathrm{E}-06$ & 0.4741521 & 301.61224 \\
\hline & 70 & 0.00496417 & 18 & 0.0012765 & 1.27977E-06 & 0.6453737 & 111.43409 \\
\hline & & & & & & & \\
\hline
\end{tabular}


Table D1 (continue)

\begin{tabular}{|c|c|c|c|c|c|c|c|c|c|c|}
\hline & & & & & & & & & & \\
\hline & & & & & & & & & & \\
\hline & & & & & & & & & & \\
\hline & & & & & & & & & & \\
\hline & & & & & & & & & & \\
\hline & & & & & & & & & & \\
\hline & & & & & & & & & & \\
\hline & & & & & & & & & & \\
\hline & & & & & & & & & & \\
\hline & & & & & & & & & & \\
\hline & & & & & & & & & & \\
\hline & & & & & & & & & & \\
\hline & & & & & & & & & & \\
\hline & & & & & & & & & & \\
\hline & & & & & & & & & & \\
\hline & & & & & & & & & & \\
\hline Angle & & cos(angle) & F(I) & F(ST) & $\mathrm{F}(\mathrm{G})$ & $\mathrm{F}(1)$ & Sigma11 & Sigma(11-22) & Exten. Visc. & Rela Visc. \\
\hline $\begin{array}{l}\text { (degree) } \\
\text { (n) }\end{array}$ & (radian) & $(-)$ & (N) & (N) & $(\mathrm{N})$ & $(\mathrm{N})$ & (pa) & (pa) & (pas) & $(-)$ \\
\hline 2.6 & 0.045379 & 0.998971 & & & & & & & & \\
\hline 3.4 & 0.059341 & 0.99824 & $-6.67 \mathrm{E}-05$ & $-4.73 \mathrm{E}-05$ & 7.5E-05 & 0.0002934 & 36.68323 & 80.55318216 & 6.6228479 & 105.1246 \\
\hline 4.6 & 0.080285 & 0.996779 & $-6.17 \mathrm{E}-05$ & $-6.38 \mathrm{E}-05$ & 0.000144 & 0.0002463 & 32.20285 & 77.06985506 & 7.1546717 & 113.5662 \\
\hline 6.6 & 0.115192 & 0.993373 & $-5.08 E-05$ & $-9.71 \mathrm{E}-05$ & 0.000208 & 0.0002264 & 32.49619 & 79.49971812 & 3.8262569 & 60.73424 \\
\hline 11.3 & 0.197223 & 0.980615 & $-3.1 \mathrm{E}-05$ & -0.000151 & 0.000265 & 0.0002435 & 40.5288 & 91.14798074 & 1.3051954 & 20.71739 \\
\hline 20.6 & 0.359539 & 0.936059 & 5.249E-05 & -0.000295 & 0.000307 & 0.0004282 & 112.8096 & 176.4918027 & 0.7435166 & 11.80185 \\
\hline 48.4 & 0.844741 & 0.663925 & 0.0003199 & -0.00053 & 0.000331 & 0.000907 & 520.6981 & 614.7051641 & 2.0380644 & 32.35023 \\
\hline 0 & 0 & 1 & 0.0004984 & -0.000467 & 0.000344 & 0.001009 & 788.4111 & 898.0859905 & & \\
\hline & & & & & & & & Ave. & 3.6150921 & 57.38241 \\
\hline
\end{tabular}




\begin{tabular}{|c|c|c|c|c|c|c|c|}
\hline \multicolumn{8}{|c|}{$\begin{array}{c}\text { Table D2 Calculation detail for } 200 \text { ppm F20W suspension in corn syrup solution (25\% } \\
\text { water in weight) }\end{array}$} \\
\hline & Thin Wall Tube & Gauge & 10-12 for $200 \mathrm{ppm}$ & F20W at $25 \%$ & com syrup & & \\
\hline & & & & density $(\mathrm{g} / \mathrm{ml})$ & 1.262 & & \\
\hline & & & shear visoosity & (pas) & 0.063 & & \\
\hline & & & suface tension ooe. & $(\mathrm{N} / \mathrm{m})$ & 0.07 & & \\
\hline & flow rate & measure & & & & & \\
\hline & & & & & & read. diff. & pre. Diff. \\
\hline & flowvolume (ml) & time (s) & flow rate $(\mathrm{ml} / \mathrm{s})$ & flow rate $(\mathrm{m} 3 / \mathrm{s})$ & & $(\mathrm{mmH} 2 \mathrm{O})$ & (pa) \\
\hline 1 & 9.9 & 12.59 & 0.786338364 & $7.86338 \mathrm{E}-07$ & & 2 & 21.30912 \\
\hline 2 & 10 & 12.69 & 0.788022065 & $7.88022 \mathrm{E}-07$ & & (degree 5 & for U tube) \\
\hline 3 & 9.7 & 12.32 & 0.787337662 & 7.87338E-07 & & & \\
\hline ave. & & & & 7.87233E-07 & & & \\
\hline & $\mathrm{F}(\mathrm{O})=(\mathrm{PO}-\mathrm{P} 1)^{*} \mathrm{AO}(\mathrm{N})$ & 0.00019 & & & & & \\
\hline & Sec. term in F(I) & $1.3 \mathrm{E}-07$ & $\left(16^{*} \mathrm{Q}^{\wedge} 2 /\left(3^{*}\right.\right.$ pai*d^2)) & & & & \\
\hline & & & & & & & \\
\hline & Upp. Tub. OD (m) & 0.0034 & ID (m) & 0.0028956 & & & \\
\hline & Dist. From exit of Upp. Capilary & & Filament diameter & & Cross sec. area & Velocity (v) & $\mathrm{dv} / \mathrm{dz}$ \\
\hline & $(-)$ & (m) & $(-)(\mathrm{z})$ & (m) & $\left(\mathrm{m}^{\wedge} 2\right)$ & $(\mathrm{m} / \mathrm{s})$ & $(1 / \mathrm{s})$ \\
\hline & 0 & 0 & 46 & 0.003404 & 9.1006E-06 & 0.0865034 & \\
\hline & 15 & 0.00111 & 41 & 0.003034 & $7.22973 \mathrm{E}-06$ & 0.1088883 & 18.13353 \\
\hline & 30 & 0.00222 & 38 & 0.002812 & $6.21043 \mathrm{E}-06$ & 0.1267599 & 18.25816 \\
\hline & 45 & 0.00333 & 35 & 0.00259 & $5.26854 \mathrm{E}-06$ & 0.1494214 & 18.61355 \\
\hline & 60 & 0.00444 & 33 & 0.002442 & $4.68363 \mathrm{E}-06$ & 0.1680819 & 18.49015 \\
\hline & 75 & 0.00555 & 31 & 0.002294 & $4.13312 \mathrm{E}-06$ & 0.1904696 & 56.20903 \\
\hline & 90 & 0.00666 & 25 & 0.00185 & $2.68803 E-06$ & 0.292866 & 208.4798 \\
\hline & 109 & 0.00807 & 16 & 0.001184 & $1.10102 \mathrm{E}-06$ & 0.7150049 & 43.97387 \\
\hline
\end{tabular}




\begin{tabular}{|c|c|c|c|c|c|c|c|c|c|c|}
\hline \multicolumn{11}{|c|}{ Table D2 (continue) } \\
\hline & & & & & & & & & & \\
\hline & & & & & & & & & & \\
\hline & & & & & & & & & & \\
\hline & & & & & & & & & & \\
\hline & & & & & & & & & & \\
\hline & & & & & & & & & & \\
\hline & & & & & & & & & & \\
\hline & & & & & & & & & & \\
\hline & & & & & & & & & & \\
\hline & & & & & & & & & & \\
\hline & & & & & & & & & & \\
\hline & & & & & & & & & & \\
\hline & & & & & & & & & & \\
\hline & & & & & & & & & & \\
\hline & & & & & & & & & & \\
\hline Angle & & oos(angle) & F(I) & $\mathrm{F}(\mathrm{ST})$ & $\mathrm{F}(\mathrm{G})$ & $F(1)$ & Sigma11 & Sigma(11-22) & Exten. Visc. & Rela Visc. \\
\hline (degree) & (radian) & $(-)$ & (N) & (N) & $(\mathrm{N})$ & (N) & (pa) & (pa) & (pas) & $(-)$ \\
\hline 2.6 & 0.045379 & 0.9989706 & & & & & & & & \\
\hline 4.8 & 0.083776 & 0.9964928 & 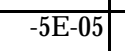 & $-8 \mathrm{E}-05$ & 0.000112 & 0.0001147 & 15.86927 & 62.01297354 & 3.4197957 & 54.28247 \\
\hline 5.2 & 0.090757 & 0.9958844 & $-3 \mathrm{E}-05$ & \begin{tabular}{|c|c|c|}
-0.0001 \\
\end{tabular} & 0.000204 & 8.925E-05 & 14.37135 & 64.15798076 & 3.5139345 & 55.77674 \\
\hline 2.7 & 0.047124 & 0.9988899 & $\begin{array}{ll}-1 \mathrm{E}-05 \\
\end{array}$ & \begin{tabular}{c|c|}
-0.0002 \\
\end{tabular} & 0.000283 & 7.989E-05 & 15.16365 & 69.21770502 & 3.7186725 & 59.02655 \\
\hline 1.5 & 0.02618 & 0.9996573 & 8.6E-06 & -0.0002 & 0.000351 & $6.219 \mathrm{E}-05$ & 13.27729 & 70.60735067 & 3.8186468 & 60.61344 \\
\hline 9.5 & 0.165807 & 0.9862855 & 3.1E-05 & \begin{tabular}{|c|c|}
-0.0003 \\
\end{tabular} & 0.000412 & 6.317E-05 & 15.28354 & 76.31230919 & 1.3576522 & 21.55004 \\
\hline 12.1 & 0.211185 & 0.9777831 & 0.00013 & -0.0004 & 0.000458 & 0.0002181 & 81.15439 & 156.8300702 & 0.7522553 & 11.94056 \\
\hline 8.1 & 0.141372 & 0.9900236 & 0.00055 & -0.0005 & 0.00049 & 0.0007456 & 677.1819 & 795.4251618 & & \\
\hline & & & & & & & & Ave. & 2.7634928 & 43.86497 \\
\hline
\end{tabular}




\begin{tabular}{|c|c|c|c|c|c|c|c|}
\hline \multicolumn{8}{|c|}{$\begin{array}{c}\text { Table D3 Calculation detail for } 200 \text { ppm F25W suspension in corn syrup solution (25\% } \\
\text { water in weight) }\end{array}$} \\
\hline & Thin Wall Tube & Gauge & 10-12 for 200ppm & F25W at 25\% & oom syrup & & \\
\hline & & & & density $(\mathrm{g} / \mathrm{ml})$ & 1.262 & & \\
\hline & & & shear viscosity & (pas) & 0.063 & & \\
\hline & & & suface tension coe. & $(\mathrm{N} / \mathrm{m})$ & 0.07 & & \\
\hline & flow rate & measure & & & & & \\
\hline & & & & & & read. diff. & pre. Diff. \\
\hline & flow volume (ml) & time (s) & flow rate $(\mathrm{m} / \mathrm{s})$ & flow rate $(\mathrm{m} 3 / \mathrm{s})$ & & $(\mathrm{mmH} 2 \mathrm{O})$ & (pa) \\
\hline 1 & 9.73 & 11.56 & 0.841695502 & 8.41696E-07 & & 1.5 & 15.98184 \\
\hline 2 & 9.8 & 11.76 & 0.833333333 & 8.33333E-07 & & (degree 5 & for U tube) \\
\hline 3 & 10 & 11.91 & 0.839630563 & 8.39631E-07 & & & \\
\hline ave. & & & & $8.3822 \mathrm{E}-07$ & & & \\
\hline & $\mathrm{F}(0)=(\mathrm{PO}-\mathrm{P} 1)^{*} \mathrm{AO}(\mathrm{N})$ & 0.0001454 & & & & & \\
\hline & Sec. term in F(I) & $1.423 \mathrm{E}-07$ & $\left(16^{*} \mathrm{Q} \wedge 2 /\left(3^{*}\right.\right.$ pai*d^2)) & & & & \\
\hline & & & & & & & \\
\hline & Upp. Tub. OD (m) & 0.0034036 & ID (m) & 0.0028956 & & & \\
\hline & Dist. From exit of Upp. Capilary & & Filament diameter & & Cross sec. area & Velocity (v) & $\mathrm{dv} / \mathrm{dz}$ \\
\hline & $(-)$ & (m) & $(-)(\mathrm{z})$ & (m) & $\left(\mathrm{m}^{\wedge} 2\right)$ & $(\mathrm{m} / \mathrm{s})$ & $(1 / \mathrm{s})$ \\
\hline & 0 & 0 & 46 & 0.003404 & 9.1006E-06 & 0.092106 & \\
\hline & 15 & 0.00111 & 41 & 0.003034 & $7.22973 \mathrm{E}-06$ & 0.1159407 & 16.23016 \\
\hline & 30 & 0.00222 & 39 & 0.002886 & $6.54159 \mathrm{E}-06$ & 0.128137 & 15.51453 \\
\hline & 45 & 0.00333 & 36 & 0.002664 & $5.5739 \mathrm{E}-06$ & 0.150383 & 18.22454 \\
\hline & 60 & 0.00444 & 34 & 0.002516 & $4.97178 \mathrm{E}-06$ & 0.1685954 & 23.61387 \\
\hline & 75 & 0.00555 & 31 & 0.002294 & $4.13312 \mathrm{E}-06$ & 0.2028058 & 76.47128 \\
\hline & 90 & 0.00666 & 24 & 0.001776 & $2.47729 \mathrm{E}-06$ & 0.3383617 & 185.7997 \\
\hline & 104 & 0.007696 & 18 & 0.001332 & $1.39348 \mathrm{E}-06$ & 0.6015319 & 50.80506 \\
\hline & & & & & & & \\
\hline
\end{tabular}




\begin{tabular}{|c|c|c|c|c|c|c|c|c|c|c|}
\hline \multicolumn{11}{|c|}{ Table D3 (continue) } \\
\hline & & & & & & & & & & \\
\hline & & & & & & & & & & \\
\hline & & & & & & & & & & \\
\hline & & & & & & & & & & \\
\hline & & & & & & & & & & \\
\hline & & & & & & & & & & \\
\hline & & & & & & & & & & \\
\hline & & & & & & & & & & \\
\hline & & & & & & & & & & \\
\hline & & & & & & & & & & \\
\hline & & & & & & & & & & \\
\hline & & & & & & & & & & \\
\hline & & & & & & & & & & \\
\hline & & & & & & & & & & \\
\hline & & & & & & & & & & \\
\hline & & & & & & & & & & \\
\hline Angle & & cos(angle) & $\mathrm{F}(\mathrm{I})$ & F(ST) & F(G) & $\mathrm{F}(1)$ & Sigma11 & Sigma(11-22) & Exten. Visc. & Rela Visc. \\
\hline (degrøe) & (radian) & $(-)$ & (N) & (N) & (N) & (N) & (pa) & (pa) & (pas) & $(-)$ \\
\hline 7.6 & 0.13265 & 0.991215 & & & & & & & & \\
\hline 3 & 0.05236 & 0.99863 & $-5.689 \mathrm{E}-05$ & \begin{tabular}{l|}
$-7.571 \mathrm{E}-05$ \\
\end{tabular} & 0.00011196 & 5.23E-05 & 7.23402 & 53.37772465 & 3.2887985 & 52.20315 \\
\hline 2.7 & 0.04712 & 0.99889 & $-4.398 \mathrm{E}-05$ & -0.000108 & 0.000206543 & $2.9551 \mathrm{E}-06$ & 0.45174 & 48.96178895 & 3.155866 & 50.093111 \\
\hline 3.4 & 0.05934 & 0.99824 & $-2.045 \mathrm{E}-05$ & -0.0001572 & 0.000289701 & $-7.522 \mathrm{E}-06$ & \begin{tabular}{|l|}
-1.34958 \\
\end{tabular} & 51.20297449 & 2.8095623 & 44.596227 \\
\hline 5.2 & 0.09076 & 0.995884 & $-1.186 \mathrm{E}-06$ & -0.000191 & 0.000362121 & $-2688 \mathrm{E}-05$ & -5.4073 & 50.23657644 & 2.1274184 & 33.768546 \\
\hline 12.1 & 0.21119 & \begin{tabular}{l|}
0.977783 \\
\end{tabular} & 3.5E-05 & -0.0002487 & 0.000424592 & 4.5848E-06 & 1.109281 & 62.13805176 & 0.8125671 & 12.897891 \\
\hline 17.1 & 0.29845 & 0.955793 & 0.0001784 & -0.0003687 & 0.00046953 & 0.00022302 & 90.0238 & 168.8526321 & 0.9087885 & 14.425215 \\
\hline 5.2 & 0.09076 & 0.995884 & 0.0004568 & -0.0004503 & 0.000494022 & 0.00055849 & 400.7921 & 505.8972539 & & \\
\hline & & & & & & & & Ave. & 2.1838335 & 34.664023 \\
\hline
\end{tabular}

